

\section{DYNAMICS OF VARIABLE MASS SYSTEMS}

\section{ABSTRACT}

This report presents the results of an investigation of the effects of mass loss on the attitude behavior of spinning bodies in flight. The principal goal is to determine whether there are circumstances under which the motion of variable mass systems can become unstable in the sense that their transverse angular velocities become unbounded. Obviously, results from a study if this kind would find immediate application in the aerospace field.

The first part of this study features a complete and mathematically rigorous derivation of a set of equations that govern both the translational and rotational motions of general variable mass systems. The remainder of the study is then devoted to the application of the equations obtained to a systematic investigation of the effect of various mass loss scenarios on the dynamics of increasingly complex models of variable mass systems.

It is found that mass loss can have a major impact on the dynamics of mechanical systems, including a possible change in the systems stability picture. Factors such as nozzle geometry, combustion chamber geometry, propellant's initial shape, size and relative mass, and propellant location can all have important influences on the system's dynamic behavior. The relative importance of these parameters on system motion are quantified in a way that is useful for design purposes. 


\section{ACKNOWLEDGMENT}

Although there is only one official author listed on the cover of this document, the material contained in this report is based on research work performed by several graduate students who were supported under this NASA grant. Hence, I wish to acknowledge the contributions of the following individuals: Dr. Song-Min Wang; Dr. Tai-Chien Mao, whose Ph.D dissertation work was completely supported by this grant; Mr. Enrique Cervantes, whose M.S thesis work was based on this topic and was supported by the grant; Mrs Asayo Harvey, whose M.S thesis was partially supported by this grant; and Mr. Jason Mudge, whose $\mathrm{Ph} . \mathrm{D}$ research work was supported by this grant until the expiration of the grant. Each of these individuals made important contributions to the final outcome of this research project.

I take this opportunity to express my immense gratitude to the NASA Technical Officer in charge of this project, Dr. Kajal Gupta of NASA Dryden, for his frequent and invaluable advice, support and inspiration throughout the duration of the project. His kindness has had a profound positive influence not only on the outcome of this work but on my whole career. I also wish to thank Mr. Steven Yee, the Grants Officer, for his patience, understanding, and assistance with paperwork on several occasions. Finally, I am most grateful to NASA for the generous support of my research effort through this grant No. NAG-2-4003. 


\section{TABLE OF CONTENTS}

Abstract ii

Acknowledgments

Chapter 1 Introduction 1

1.1 Background 1

1.2 Early Studies of Variable Mass systems 2

1.3 The Star 48 Problem $\quad . .4$

1.4 This Work 5

$\begin{array}{lll}\text { Chapter } 2 & \text { Equations of Motion of Variable Mass Systems } & 7\end{array}$

2.1 Model Description 7

2.2 Equation Formulation Strategy 9

2.3 Dynamical Equations 13

2.3.1 Useful Kinematical Quantities 14

2.3.2 Generalized Inertia Forces 18

2.3.3 Generalized Active Forces 21

2.3.4 Equations of Motion 23

2.3.5 Another Form of the Equation of Attitude Motion 28

Chapter 3 Effects of Mass Variation 31

3.1 Translational Motion 31

3.2 Rotational Motion 34

Chapter 4 Attitude Motions of a Variable Mass Cylinder 39

4.1 Introduction 39

4.2 Attitude Equations for the Cylinder ..

4.3 Attitude Stability 43

4.3.1 The Uniform Burning Cylinder 47

4.3.2 The End Burning Cylinder 48

4.3.3 Radial Burn $\quad 52$

4.3.4 Centripetal Burn 60

4.4 Time dependence of Motion and inertia Properties 64

4.4.1 End Burning Case 65

4.4.2 Centrifugal Burn 68

$\begin{array}{lll}\text { 4.4.3 Centripetal Burn } & 71\end{array}$ 
Chapter 5 Motion of Axisymmetric Bodies with Mass Loss

5.1 Governing Equations 75

5.2 Non-Dimensional Equations of Motion 80

$\begin{array}{llll}5.3 & \text { Centrifugal Burn } & . & 87\end{array}$

$\begin{array}{lll}\text { 5.3.1 Spin Rate Analysis } & 92\end{array}$

5.3.2 Transverse Rate Analysis 94

5.4 Uniform Burn

5.4.1 Spin Rate Analysis 101

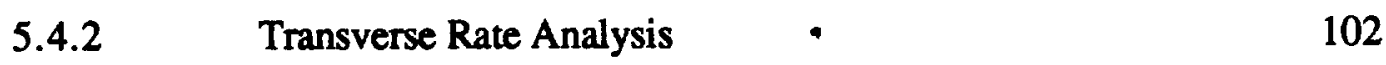

Chapter 6 Forced Motion of Axisymmetric Systems 104

6.1 Equations of Attitude Motion 105

6.2 Change of the Independent Variable 110

6.3 Special Case - Rigid Body Motion 113

$\begin{array}{lll}\text { Chapter } 7 & \text { Conclusion } & 116\end{array}$

$\begin{array}{ll}\text { References and Bibliography } & 118\end{array}$ 


\section{CHAPTER 1}

\section{INTRODUCTION}

\subsection{Background}

Studies of free and forced motions of spinning rigid bodies of various geometries have been, and continue to be documented in great detail in the literature. Such studies have led to the development of important scientific instruments (gyroscopes, etc.) and to the concept of spin stabilization of modern spacecraft. By contrast, variable mass systems have received relatively little attention in the literature, though they play an equally important role in modern technology, especially in space flight. The expression "variable mass system," as used in the context of this document, refers to mechanical systems that lose and/or gain mass while in motion. Examples of such devices abound in the engineering literature. They include complex systems such as aircraft, rockets, and moving robots picking up or letting go of objects, as well as simpler systems such as water sprinkler systems or an inflated balloon with air loss through one or more holes.

Variable mass systems can be divided into two classes: those with continuous mass variation and those with discrete mass variation. Rockets, for example, fall in the continuous variable mass class; and, robots picking up or releasing objects, or a moving vehicle dropping off some of its payload in discrete chunks, fall in the discrete variable mass system class. Because systems with discrete mass variation can be analyzed using well known principles of multi-rigid-body dynamics, the focus of the analysis presented in this study is on systems with continuously varying mass. 
It is clear from intuition that when the net change in mass of a system, as well as its mass variation rate are small, it is unnecessary to account for the change in mass in the study of the system's motion. For example, automobiles are in fact variable mass systems, yet, no one takes mass variation into account in handling and performance studies of ordinary automobiles. The reason is that the rate of mass variation is viewed as negligible - and rightly so. On the other hand, a system that undergoes a substantial change in mass, especially if this occurs in a short period of time, will definitely require that mass variation be accounted for in the study of its motion; otherwise, any predicted response of the system will be far removed from its true behavior. The focus of this study is on the determination of the impact of mass variation on the dynamic behavior of systems with substantial change in mass

\subsection{Early Studies of Variable Mass Systems}

Scientific study of variable mass systems has been in progress for more than two hundred years; so developments in this field have a long though sporadic history. The earliest recorded work on the dynamics of bodies with varying mass was performed in the 18 th century by Bernoulli (1738). He was then studying the forces acting on a liquid jet propelled hydroreactive ship - an ancient application of the principle of jet propulsion. He actually derived what may be referred to as the equation of motion in this special case. The Czech scientist and inventor, George von Buquoy (1781-1851), was the first to pose the general problem of the dynamics of systems with varying mass. In 1812 , he obtained his "motion formula" for such systems, and went on to solve a large number of examples based on his formula. von Buquoy's work can be said to mark the birth of the theory of the dynamics of systems with varying mass. In the mean time, William Moore worked out his mathematical theory of rocket motion in England in 1813, and, in 1819, Poisson took a 
rather modern approach and derived the equations of motion of variable mass systems based on Lagrange's general formula. In their book published in 1856, Tait and Steele included a section on mass variation. They postulated that mass variation produced small continuous impacts or impulsive forces on systems, and thus resulted in continuous change of velocity. This work was followed, several years later, by that of Meshcherskii, whose work spanned the period 1897 to 1904 . He essentially laid the foundation for the development of variable mass dynamics as a special discipline of mechanics. He devoted his 160 page master's thesis to exploring a large array of issues relevant to variable mass dynamics - from the derivation of equations of motion to the solution of a series of problems in the field. All of these early investigations of variable mass systems were limited in one way. They were only concerned with the study of the translational motion of such systems. The issue of rotational motion of such systems was not addressed until the mid 1940's.

The second world war brought with it a resurgence of interest and activity in the dynamics of variable mass systems, mostly in connection with rocketry. At this time, translational motion of such systems was relatively well understood and the main focus of research in variable mass dynamics began to shift to the attitude motion of such systems. Some of the scientific giants of this new era include Rosser et al. (1947), Gantmacher and Levin (1947), Rankin (1949), Ellis and McArthur (1959). The equations of rotational motion derived by these investigators are quite similar, and have forms similar to Euler's equations for rigid bodies, with extra terms that account for mass variability. Thomson stands out as a major contributor to this field through his book (1961) and the companion papers $(1965,1966)$. He derived several versions of the equations of motion of variable mass systems and his work gives a great deal of physical insight into the behavior of rockets. In his study of the transverse attitude motion of a non-spinning axisymmetric rocket, he showed that transverse rotation rate depends on the ratio of the distance of the system mass center from nozzle exit, to the transverse radius of gyration of the rocket. If 
this ratio is greater than one (the most common case), the transverse angular velocity decreases with time; and when the ratio is less than one, the transverse angular velocity increases with time. Warner and Snyder (1968) brought some refinements to Thomson's work and pointed out how various simplifying assumptions can lead to drastically different motion predictions. Meirovitch (1970) moved work on variable mass systems one step further by considering the impact of mass variation on variable mass rockets.

\subsection{The Star 48 Problem}

Flaws in current understanding of the dynamics of variable mass systems was brought to light in the early 80 's, when several space missions with upper stages powered by the Star $\mathbf{4 8}$ solid rocket motor were observed to exhibit anomalous behavior. Unexpected and unexplainable rapid growth in cone angle occurred near the end of the motor burn. The output of a typical rate gyro mounted on one of such flights is shown in Fig. 1. We note from this figure that the flight is uneventful until about two thirds into the motor burn, when there begins an exponential growth in transverse rate, and thus in nutation angle. The Star $\mathbf{4 8}$ is the first solid rocket motor known to produce such anomaly, and it differs from its predecessors mainly in its much larger size and the existence of a submerged nozzle construction.

The Star 48 problem sparked another flurry of investigations [ Eke (1983), Meyer (1983), Mingori and Yam (1986), Flandro et al. (1987), Cochran and Kang (1991)] into the behavior of variable mass systems, and is one of the main factors that motivated this work.

In his Ph. D dissertation work, part of which was supported by this project, Wang (1993) [ see also Eke and Wang (1995)] modeled rocket type variable mass systems as a 
simple cylinder of varying mass, and produced elaborate closed-form expressions that describe the attitude motion of such systems for various burn geometries. The study showed that certain propellant burn scenarios can actually cause the transverse rates of short and large rocket systems to diverge in a manner similar to that observed on the Star 48 flights.

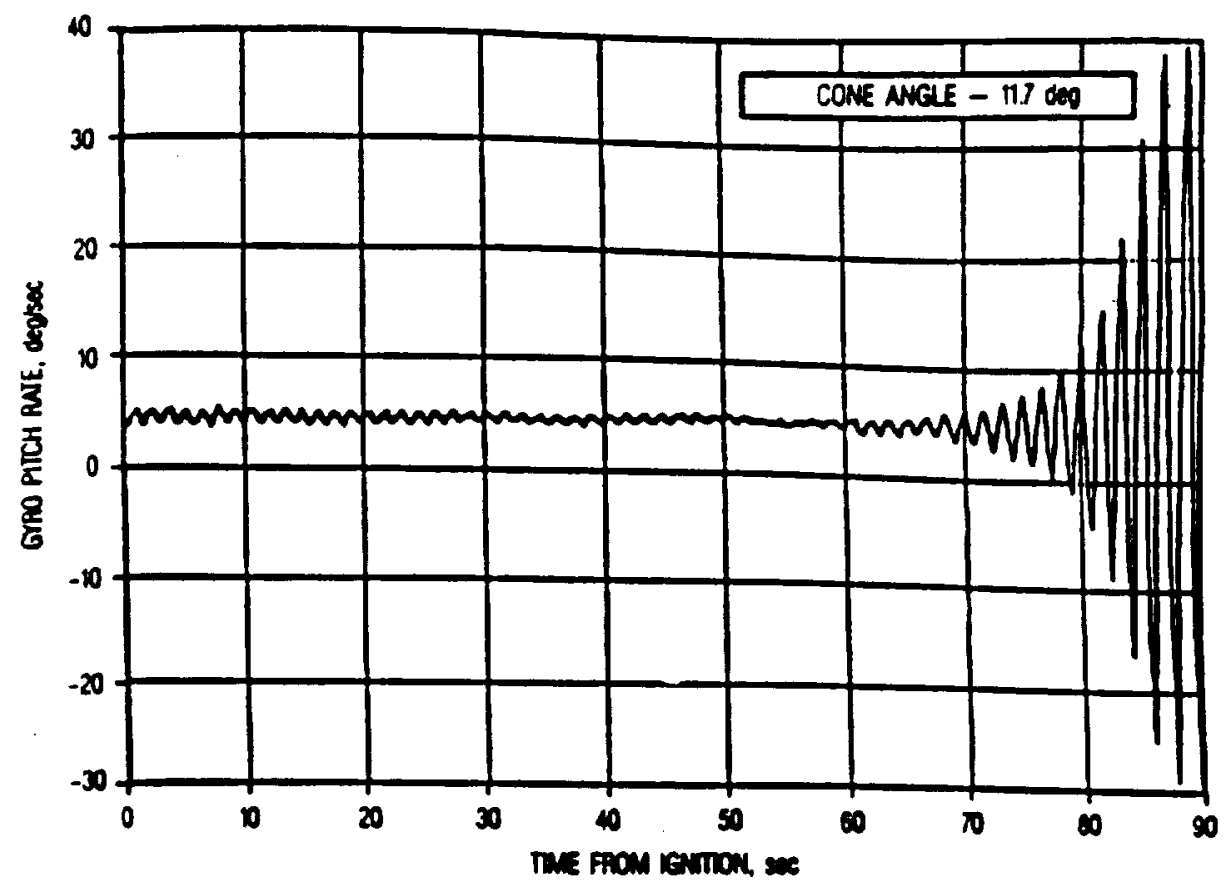

Fig. 1.1 Transverse Rate vs. Time for a Typical Star 48 Flight

\subsection{This Work}

This study is an extension of Wang's work. Like Wang's work (1993), this study utilizes modern mathematical tools in the study of the dynamics of variable mass systems, with the general intent of making valuable contributions to the field and enhancing current understanding of the dynamic behavior of such systems. 
Most investigators that have attempted the derivation of dynamical equations for variable mass systems have relied heavily on heuristics. In the next chapter, we present complete and mathematically rigorous derivations of both the translational and rotational equations of motion of variable mass systems. The remainder of the study applies the equations of chapter 2 to increasingly complex models of various classes of variable mass systems, extracting and presenting a wealth of new information on the attitude dynamics of such systems.

Throughout this document, equations and figures are numbered in the form (a.b), where the first number $a$ represents the chapter in which the item appears, and the second number $b$ is the actual item number within the chapter. To refer to an item, the number $b$ is used if the referencing is done in the same chapter in which the item appears; otherwise, the full label $(a . b)$ is utilized. 


\section{CHAPTER 2}

\section{EQUATIONS OF MOTION OF VARIABLE MASS SYSTEMS}

This chapter begins with a description of the model used to characterize variable mass systems in this study. This model is general enough to represent a wide variety of physical systems that gain or lose mass while subjected to general three-dimensional motion. The complete equations of motion for both rotational and translational motion of such systems are derived using one of the methods of analytical dynamics - Kane's formalism (Kane and Levinson 1985). The merit of this approach is its efficiency. It produces the equations of transitional and rotational motion in one mathematically rigorous step, and makes it possible to clarify a lot of conceptual issues in the derivation, that have been very difficult to do in previous work. The equations of motion that are derived are then compared with those obtained by Wang (1993) and others, who used the NewtonEuler approach.

\subsection{Model Description}

The system of interest is shown, in its most general form, in Fig. 1. It is determined by the closed surface, B, and its contents. The contents of B at any given instant can be solid (R), fluid (G), or a mixture of both. B and its contents undergo general three-dimensional motion in space, and matter can flow continuously in and out of B 
during this motion. For example, parts of $\mathrm{R}$ can "dissolve" into $\mathrm{G}$ by combustion or other processes; and, some of such products of combustion can then flow across the boundary B. At any given instant of time, only the surface B and whatever happens to be inside it at the instant constitute "the system" for that instant. Thus, the system under consideration here evolves continuously, both as regards its location in space, and its material constitution - and hence its mass. We will use the symbol $S$ to designate this system.
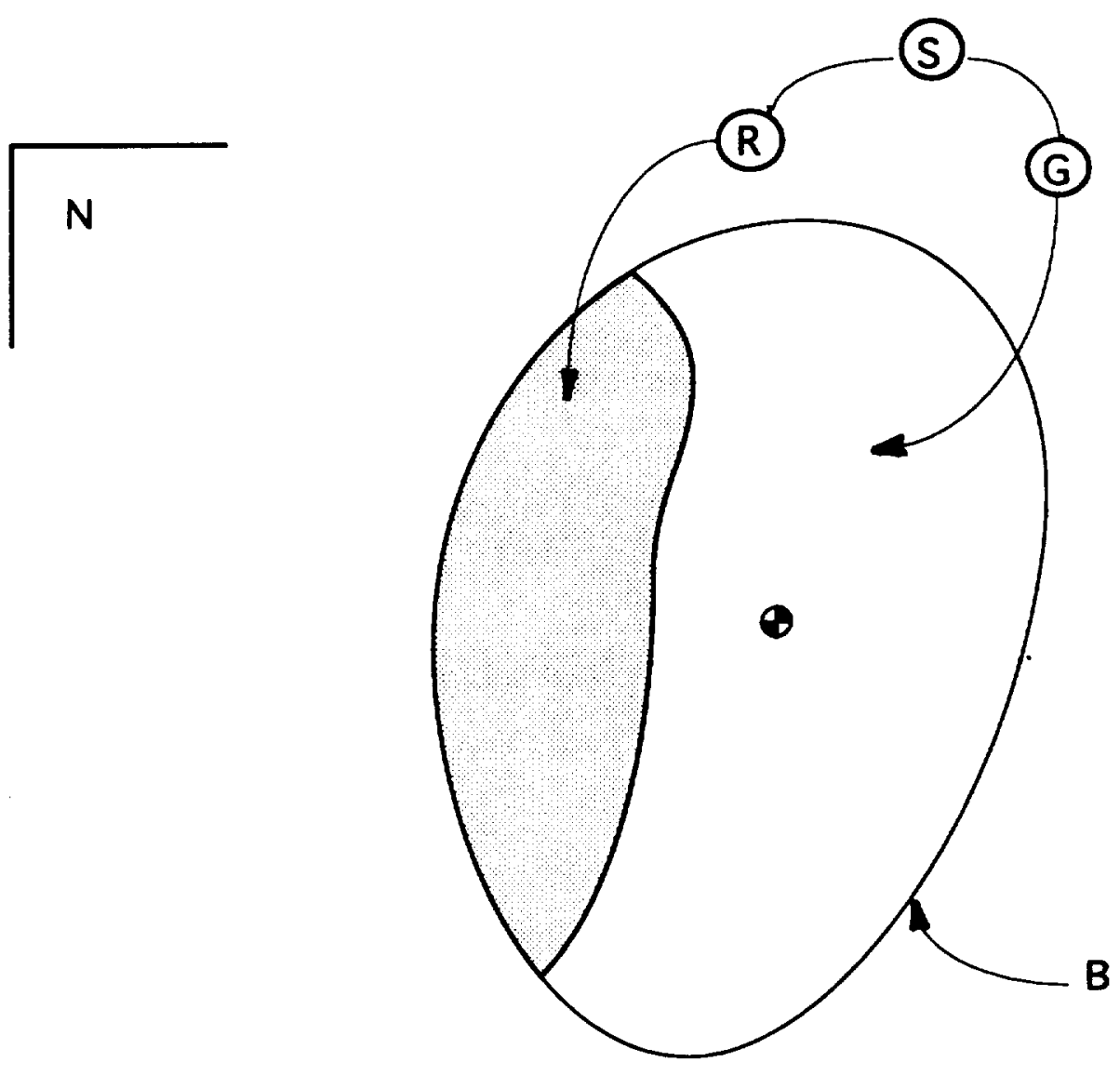

Fig. 2.1 Variable Mass System 
The derivation of equations of motion for a system such as the one described above is not as straightforward as it would normally be for a system of particles and/or rigid bodies of constant mass. The reason is that the basic principles of dynamics, such as Newton-Euler equations and Lagrange's equations are only valid when applied to a definite set of particles or rigid bodies. Two choices are then possible at this point. One, is to seek or develop new formalisms that would be valid for variable mass systems; another solution is to model variable mass systems in a way that allows them to be viewed as constant mass systems, and thus make them amenable to treatment by existing principles of dynamics. This latter approach is the choice adopted in this study.

\subsection{Equation Formulation Strategy}

Before formulating the dynamical equations for the system of Fig. 1, we start by temporarily restricting the system in some important ways. First, we assume that the closed boundary, B, of the system maintains a constant shape, and thus encloses a region of constant volume at all times. In other words, B is taken to be a rigid massless shell. As further help in the equation derivation process, we introduce the concept of constant mass systems associated with the variable mass system under study.

We consider once more the system as shown in Fig. 1, keeping in mind that the outer shell is now of constant shape. At some instant of time, $t_{1}$, there is a definite set of material particles inside B. Let us assume that this set of particles is contained in a closed elastic container, $B_{1}$, that is identical to $B$ at time $t_{t}$. In fact, we take the viewpoint that $B_{1}$ has always enclosed the exact particles that ended up in $B$ at time $t_{l}$, and that $B_{1}$ will continue to delimit these particles. Obviously, subsequent to time $t_{1}$, the shape of $B_{1}$ will deviate from that of B if it is to continue to delimit the particles that were in B at time $t_{l}$, 
since some (or even all) of these particles may have exited B. Similarly, prior to time $t_{l}$, the shape of $B_{1}$ was quite different from that of $B$, since only some, or maybe none, of the particles inside $B_{1}$ then were also inside $B$. The shape of $B_{1}$ is thus seen to vary with time, becoming identical to, and containing the same amount of matter as B at time $t_{t}$. We note however, that $B_{1}$ and its contents maintain the same mass at all times. We shall represent $B_{1}$ and its contents with the symbol $\underline{S}_{1}$, which will be referred to henceforth as the constant mass system associated with the variable mass system $S$ at time $t_{i}$. Similarly, we can define $\underline{S}_{2}, S_{3}$, etc., as constant mass systems associated with $S$ at times $t_{2}, t_{3}$, etc. Furthermore, we assume that there exists a special subset of the particles of $S$ that remains within B throughout the interval of time of interest in this study. In fact, this set is further assumed to constitute a rigid body, $R$, that is rigidly connected to $B$.

The equations of motion of any one of the constant mass systems described above can be formulated using any of the classical approaches (Newton's Second Law, Lagrange's Equations, Kane's Equations, etc.) since each system is of constant mass. Suppose, for example, that Kane's formalism is applied to the constant mass system $\underline{S}_{\mathbf{k}}$ and yields

$$
\left(F_{r}\right)_{k}+\left(F_{r}^{*}\right)_{k}=0 \quad\left(r=1,2, \ldots, n_{k}\right)
$$

where $F_{r}$ is the generalized active force on the system, $F_{r}^{*}$ is the generalized inertia force, $n_{k}$ is the number of degrees of freedom of $S_{k}$, and the subscript $k$ simply indicates that we are dealing with the system $\underline{S}_{\mathbf{4}}$. Assuming that the motion of the fluid particles of $\underline{S}_{\mathbf{k}}$ relative to the rigid part of this constant mass system is known, $\underline{\underline{S}}_{\mathbf{z}}$ has six degrees of freedom $n_{k}=6$.

Eqs. (1) have the explicit form 


$$
\dot{u}_{r}^{k}=f_{r}^{k}\left(u_{1}^{k}, \ldots, u_{6}^{k}, q_{1}^{k}, \quad, q_{6}^{k}, t\right) \quad r=1,2, \ldots, 6
$$

where $t$ is time, $q$,and $u_{r}^{k}$ are, respectively, the $r$ - th generalized coordinate and generalized speed of the constant mass system $S_{\mathbf{4}}$. In other words, the time derivative of a given generalized speed of $S_{4}$, is a function of generalized speeds and generalized coordinates of $S_{\text {, }}$ and possibly time. Eqs. (2) can be supplemented with kinematical equations of the form

$$
\dot{q}_{r}^{k}=g_{r}^{k}\left(u_{1}^{k}, \ldots, u_{6}^{k}, q_{1}^{k},, q_{6}^{k}, t\right) \quad r=1,2, \ldots, 6
$$

Eqs. (2) and (3) constitute the equations of motion of $\underline{S}_{k}$, and can be written as

$$
\dot{y}_{r}^{k}=h_{r}^{k}\left(y_{1}^{k}, \ldots, y_{12}^{k}, t\right) \quad r=1,2, \ldots, 12
$$

where $y_{i}^{\prime}$ is a generalized motion variable (generalized speed or generalized coordinate) of S.

Next, we imagine that the set of differential equations (4) is solved for $y_{i}^{k}$ as functions of time. We note then that $\left.y_{i}^{k}\right|_{t+1}$ are the generalized motion variables for the variable mass system $S$ at time $t=t_{k}$. We now consider some quantity $\underline{k}_{k}$, that characterizes the motion of $S_{\mathbf{z}}$ in some way, and is therefore obtainable from the generalized motion variables $y_{i}^{k}$, or is simply one of them. An example of a good candidate for $\underline{y}_{k}$ is the magnitude of the velocity of the mass center of $\mathbf{S}_{\mathbf{X}}$. Another example is a component of the angular velocity of the rigid body $B$ that is a part of $\underline{S}_{\mathbf{A}}$. We will call $\underline{y}_{k}$ a characteristic motion variable for $\underline{S}_{k}$. Once $y_{i}^{k}$ is known for all times, $\underline{x}_{k}$ is known, and can be plotted as a 
function of time. Now, suppose we solve Eqs. (4) for various values of $k$, and thus determine $y_{k}$ for several constant mass systems; and all of these $\underline{k}_{k}(k=1,2, \ldots, m)$ are plotted as functions of time on the same scale, but staggered for clarity as shown in Fig. 2. Now, we consider the motion characteristic, $v$, of the variable mass system $S$, that corresponds to $\underline{k}_{k}$. If, for example, $\underline{v}_{k}$ represents the speed of the mass center of $\underline{S}_{k}$, then $v$ would represent the speed of the mass center of $S$, keeping in mind that different material particles make up $S$ at different times. Because the dynamic behavior of $S$ at some instant of time $t_{j}$ is, in fact, the dynamic behavior at time $t_{j}$ of $\underline{S}_{j}$, it is clear that $v$ is given as a function of time by the curve labeled $F$ in Fig. 2; or, more precisely, the projection of $F$ onto the plane $x_{1}-t$ (see $v$ in Fig. 2). The task before us can be viewed as the determination of an efficient method for generating the differential equations whose solutions lead directly to the curve $v$ of Fig. 2 . The route to accomplishing this task will now be delineated.

We consider once more Eqs. (4), which are the equations of motion for the constant mass system $\mathbf{S}_{\mathbf{I}}$. First, we note that this equation has the same form for various values of $k$. The only items that change with $k$ are system parameters such as mass and moments of inertia. Setting $t=t_{k}$ in Eqs. (4) yields a set of algebraic equations that produce $y_{r}^{k} / t_{t=t k}$ $(r=1,2, \ldots, 12)$; these quantities are equal respectively to $y_{1}\left(t_{k}\right), y_{2}\left(t_{k}\right), \ldots y_{12}\left(t_{k}\right)$, the time derivatives of the corresponding generalized motion variables for $S$ evaluated at time $t=t_{k}$. One of these (or some function of these) represents the slope of curve $v$ of Fig. 2 at $t=t_{k}$, and is plotted as point $P_{k}$ in Fig. 3. The above process can be repeated for The system $S_{1}$ at time $t_{1}$, the system $S_{2}$ at time $t_{2}$, etc., and the relevant results are used to complete the plot of Fig. 3. The equations of curves such as $P$ in Fig. 3 are the equations of motion of the variable mass system. Since the points of such curves are generated from the equations of motion of the various constant mass systems, the equations of motion of the variable mass system $S$, have exactly the same form as the equations of motion of a typical constant mass system. However, to apply such equations correctly to variable mass systems, care must be taken to interpret mass and inertia parameters correctly. At any given instant of time, 
these parameters take on their values for the corresponding constant mass system for the instant under consideration.

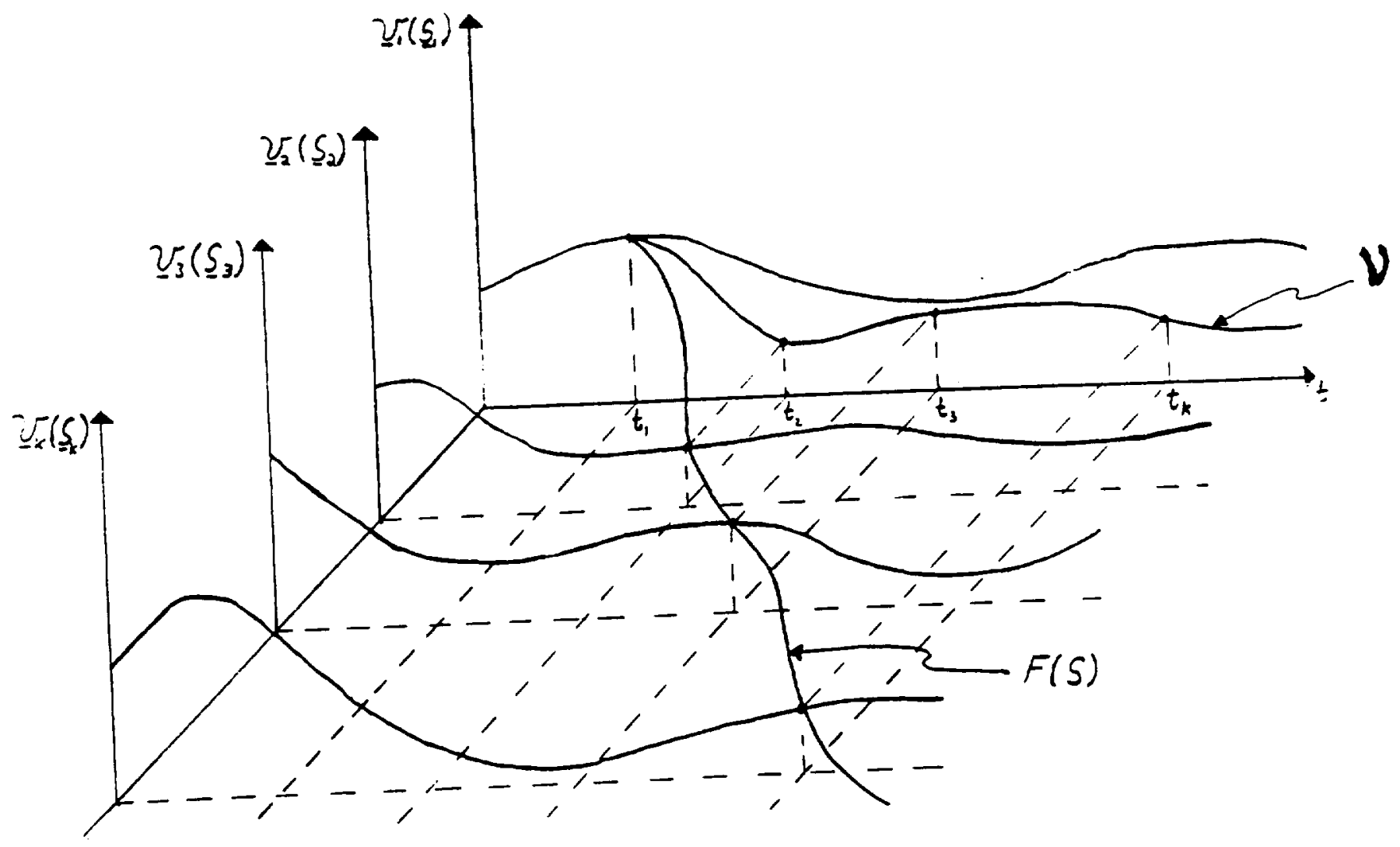

Fig. 2.2 Characteristic Variables

\subsection{Dynamical Equations}

To obtain the dynamical equations of the full variable mass system, $\mathbf{S}$, all we need to do is to derive the dynamical equations for a typical constant mass system. We will now do this using Kane's equations given in Eq. (1). 


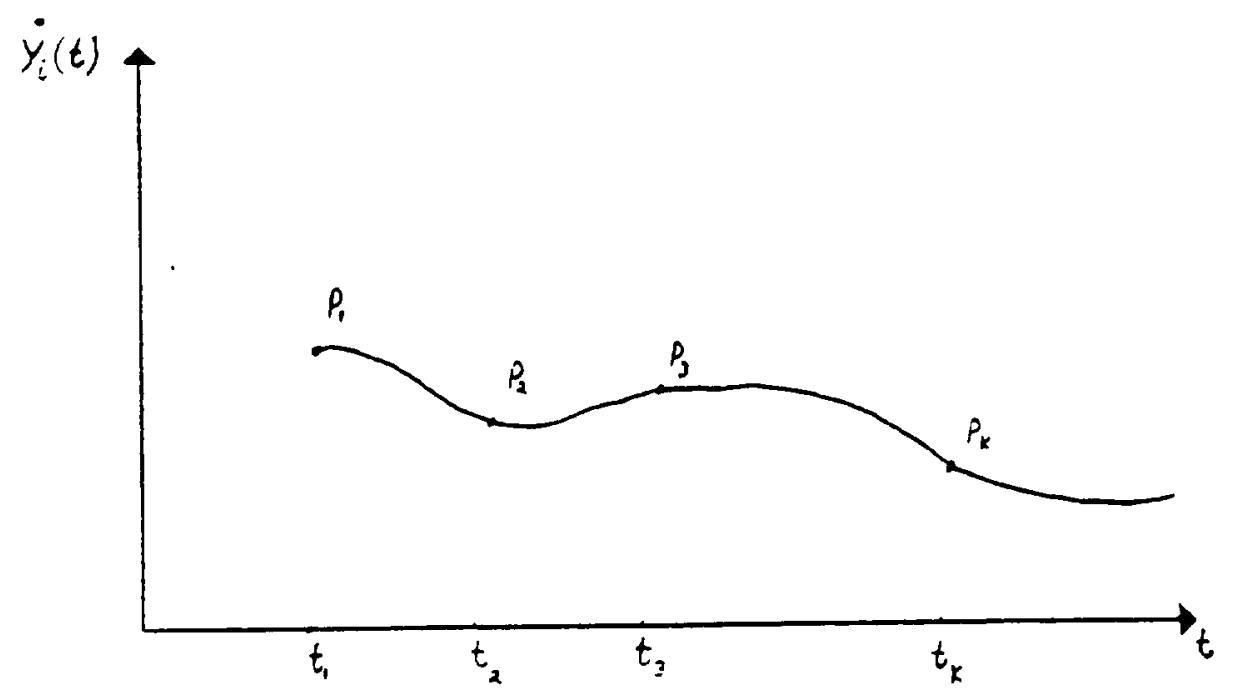

Fig. 2.3 Graph of $y_{r}(t)$ versus time

\subsubsection{Useful Kinematical Quantities}

In order to keep the mathematical developments that are going to follow relatively compact, and to avoid symbol definitions that are interspersed throughout the document, we give and define, in Table 1, a set of symbols that will be used repeatedly in the remainder of this document.

A typical constant mass system, $\mathbf{S}_{\mathbf{Z}}$, is shown in Fig. 4. A possible choice of generalized speeds, $u_{r}$, for the system is

$$
u_{r}=\left\{\begin{array}{c}
\omega \cdot b_{r}(r=1,2,3) \\
v^{*} \cdot b_{r-3}(r=4,5,6)
\end{array}\right.
$$




\section{Table 2.1 - List of Sumbols}

A symbol in boldface type signifies a vector, and a boldface symbol with a tilde above it represents a dyadic. Several of the symbols defined here are shown in Fig. 4.

- $N$ - an inertial reference frame

- $B$ - the boundary of the variable mass system

- $R$ - the solid portion of the variable mass system

- $S$ - the variable mass system enclosed by its boundary $B$

- $S^{*}$ - mass center of the system

- $O$ - an arbitrary point of $R$

- $P$ - a generic point of the system

- $v$ - velocity of $P$ in an inertial reference frame

- $\omega$ - angular velocity of $R$ in an inertial reference frame

- a - acceleration of $P$ in an inertial reference frame

- $v_{r} / a_{r}-$ velocity / acceleration of $P$ relative to $R$

$\cdot v^{o} / \mathbf{a}^{o}$ - velocity / acceleration of $O$ in an inertial frame

$\cdot \mathbf{s}^{*} / \mathrm{a}^{*}$ - velocity / acceleration of $S^{*}$ in an inertial frame

- $\mathbf{v}^{*} / \mathbf{a} \mathbf{S}^{*}$ - velocity / acceleration of $S^{*}$ relative to $R$

- $b_{\mathrm{i}}(\mathrm{i}=1,2,3)$ - a dextral set of mutually perpendicular unit vectors fixed in $R$

- $\mathbf{r}$ - position vector from $O$ to $P$ (see Fig. 3)

- $\mathbf{r}^{*}$ - position vector from $O$ to $S^{*}$ (see Fig. 3)

- a subscript, $u_{r}$, attached to any $\mathrm{v}$ or $\omega$ indicates the corresponding partial velocity

- $\mathrm{p}$ - position vector from $S^{*}$ to $P$

- $\tilde{\mathbf{I}}_{\mathrm{s}}-$ central inertia dyadic of the system 


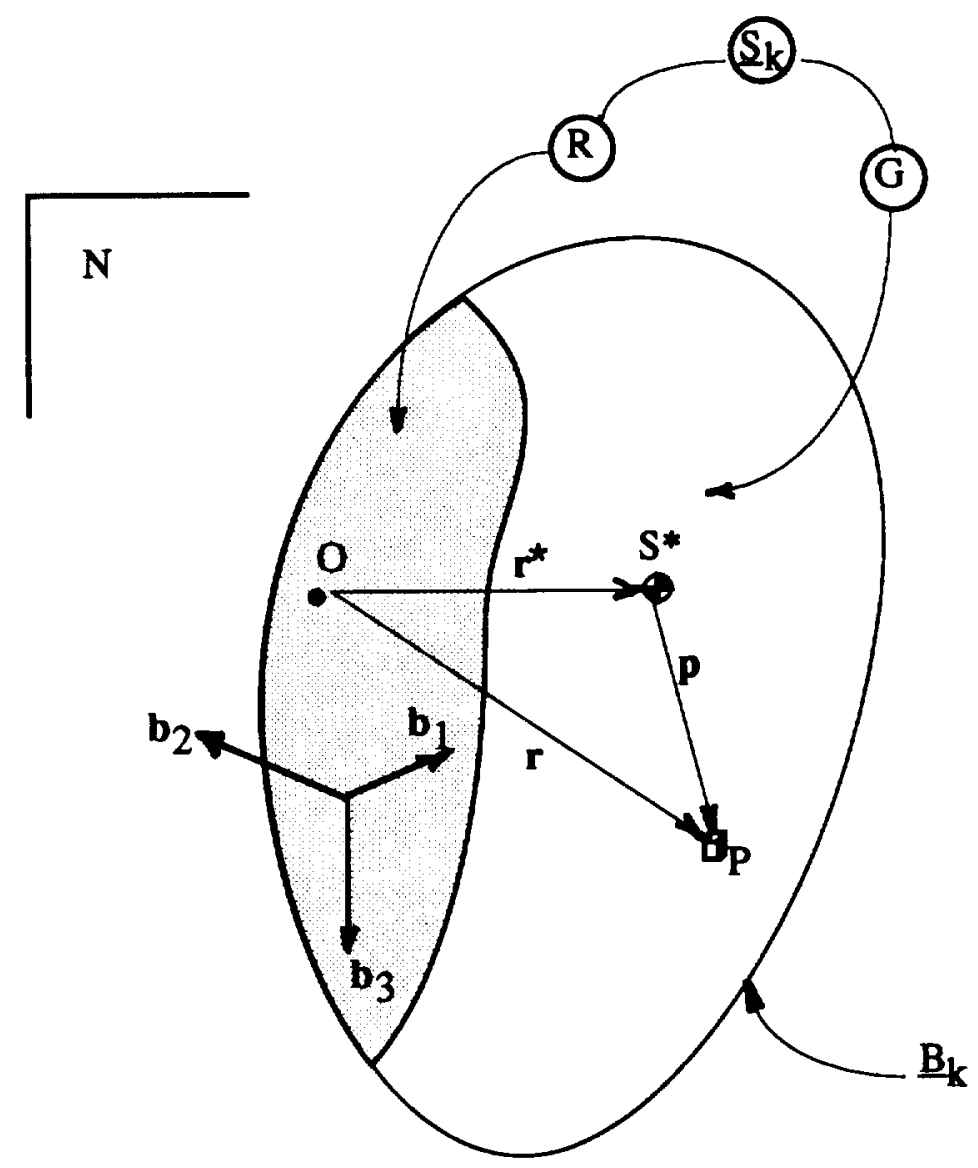

Fig. 2.4 Constant Mass System, $\mathbf{S}_{\mathbf{k}}$

By virtue of (5) above,

$$
\omega=u_{1} \mathbf{b}_{1}+u_{2} \mathbf{b}_{2}+u_{3} \mathbf{b}_{3}
$$

and

$$
v^{*}=u_{4} b_{1}+u_{5} b_{2}+u_{6} b_{3}
$$


The velocity of a generic point, $P$, of the system is

$$
\mathbf{v}=\mathbf{v}^{o}+\omega \times \mathbf{r}+\mathbf{v}_{\mathbf{r}}
$$

Because the motion of the fluid particles relative to $R$ is assumed to be described by known functions of time, the partial velocities [see Kane and Levinson (1985)] of $P$ are given by

$$
v_{u_{\mathrm{r}}}=v_{u_{\mathrm{r}}}^{0}+\omega_{u_{\mathrm{r}}} \times \mathrm{r}
$$

Now, the velocity of the system mass center can be expressed as

$$
\mathbf{v}^{*}=\mathbf{v}^{o}+\omega \times \mathbf{r}^{*}+\mathbf{v}_{r}^{s^{*}}
$$

and so, the corresponding partial velocities are

$$
\mathbf{v}_{u_{r}}^{s_{r}^{*}}=\mathbf{v}_{u_{r}}^{o}+\omega_{u_{r}} \times \mathbf{r}^{*}
$$

From (11),

$$
\mathbf{v}_{u_{r}}^{o}=\mathbf{v}_{u_{r}}^{s^{*}}-\omega_{u_{r}} \times \mathbf{r}^{*}
$$


so that (9) and (12) give

$$
\mathbf{v}_{u_{r}}=\mathbf{v}_{u_{r}}^{s^{*}}+\omega_{u_{r}} \times \mathbf{p}
$$

where $\mathrm{p}$ is the position vector from $S^{*}$ to $P$.

The acceleration of the generic point, $P$, of $S$ is

$$
\mathbf{a}=\mathbf{a}^{o}+\alpha \times \mathbf{r}+\omega \times(\omega \times \mathbf{r})+2 \omega \times \mathbf{v}_{r}+\mathbf{a}_{r}
$$

Similarly,

$$
\mathbf{a}^{s^{*}}=\mathbf{a}^{o}+\alpha \times \mathbf{r}^{*}+\omega \times\left(\omega \times \mathbf{r}^{*}\right)+2 \omega \times \mathbf{v}_{r}^{s^{*}}+a_{r}^{s^{*}}
$$

(14) and (15) can be combined to give

$$
\mathbf{a}=\mathbf{a}^{s^{*}}+\alpha \times \mathbf{p}+\omega \times(\omega \times \mathbf{p})+2 \omega \times\left(\mathbf{v}_{r}-\mathbf{v}_{r}^{s^{*}}\right)+\left(\mathbf{a}_{r}-\mathbf{a}_{r}^{s^{*}}\right)
$$

\subsubsection{Generalized Inertia Forces}

The contribution of matter contained in an elementary parallelepiped located at $P$ (see Fig. 4) to the generalized inertia forces of the system $\underline{S}_{\mathbf{x}}$ is 


$$
\left(F_{\mathrm{r}}^{*}\right)_{P}=-d m \mathbf{a} \cdot \mathbf{v}_{u_{r}}
$$

where $d m$ is the mass of matter inside the parallelepiped. (13), (16) and (17) yield

$$
\begin{aligned}
& \left(F_{r}^{*}\right)_{P}=-d m\left[\mathbf{a}^{s^{*}}+\alpha \times \mathbf{p}+\omega \times(\omega \times \mathbf{p})+2 \omega \times\left(\mathbf{v}_{r}-\mathbf{v}_{r}^{s^{*}}\right)+\left(\mathbf{a}_{r}-\mathbf{a}_{r}^{s^{*}}\right)\right] \cdot \mathbf{v}_{u_{r}}^{s^{*}} \\
& -d m\left[\mathbf{a}^{s^{*}}+\alpha \times \mathbf{p}+\omega \times(\omega \times \mathbf{p})+2 \omega \times\left(\mathbf{v}_{r}-\mathbf{v}_{r}^{s^{*}}\right)+\left(\mathbf{a}_{r}-\mathbf{a}_{r}^{s^{*}}\right)\right] \cdot\left(\omega_{u_{r}} \times \mathbf{p}\right)
\end{aligned}
$$

Hence, the generalized inertia force on $S_{4}$ corresponding to the generalized speed, $u_{r}$, is

$$
\begin{aligned}
& F_{r}^{*}=-v_{u_{r}}^{s^{*}} \cdot \int_{\Delta_{k}} \rho\left[\mathbf{a}^{s^{*}}+\alpha \times \mathbf{p}+\omega \times(\omega \times \mathbf{p})+2 \omega \times\left(\mathbf{v}_{r}-v_{r}^{s^{*}}\right)+\left(\mathbf{a}_{r}-\mathbf{a}_{r}\right)\right] d V \\
& -\omega_{u_{r}} \cdot \int_{a_{k}} \rho\left\{\mathbf{p} \times\left[\mathbf{a}^{*}+\alpha \times \mathbf{p}+\omega \times(\omega \times \mathbf{p})+2 \omega \times\left(v_{r}-v_{r}^{s^{*}}\right)+\left(\mathbf{a}_{r}-\mathbf{a}_{r} s^{*}\right)\right]\right\} d V
\end{aligned}
$$

We note here that the integrals in (19) are volume integrals that are taken over the region enclosed by $B_{\mathbf{4}}$. We recall that $B_{\mathbf{4}}$ always contains all the material particles found inside $B$ at time $t_{k}$. $\mathrm{B}_{4}$ coincides with B at the instant $t_{k}$, but is different from B at any other time.

We now return to the determination of the generalized inertia force, $F_{r}^{*}$. From Fig. 3 and the definition of mass center, 


$$
\int_{B_{k}} \mathbf{p} d m=\int_{B_{k}}\left(\mathbf{r}-\mathbf{r}^{*}\right) d m=0
$$

Similarly,

$$
\int_{B_{k}}\left(\mathbf{v}_{r}-\mathbf{v}_{r}^{s^{*}}\right) d m=\int_{B_{k}}\left(\mathbf{a}_{r}-\mathbf{a}_{r}^{s^{*}}\right) d m=0
$$

Using (20) and (21) together with the facts that

$$
\int_{B_{k}}[\mathbf{p} \times(\boldsymbol{\alpha} \times \mathbf{p})] d m=\tilde{\mathbf{I}} \cdot \boldsymbol{\alpha}
$$

and

$$
\int_{B_{k}} \mathbf{p} \times[\boldsymbol{\omega} \times(\boldsymbol{\omega} \times \mathbf{p})] d m=\int_{B_{k}} \boldsymbol{\omega} \times[\mathbf{p} \times(\boldsymbol{\omega} \times \mathbf{p})] d m=\boldsymbol{\omega} \times \tilde{\mathbf{I}} \cdot \boldsymbol{\omega}
$$

(19) is reduced to

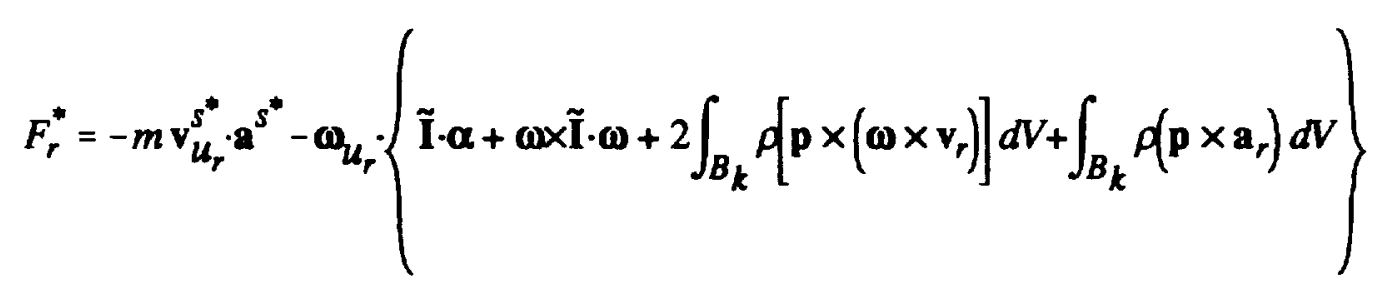

Hence, for $r=1,2$, and 3, 


$$
F_{r}^{*}=-\omega_{u_{r}} \cdot\left\{\tilde{\mathbf{I}} \cdot \boldsymbol{\alpha}+\boldsymbol{\omega} \times \tilde{\mathbf{I}} \cdot \boldsymbol{\omega}+2 \int_{B_{k}} \rho\left[\mathbf{p} \times\left(\boldsymbol{\omega} \times \mathbf{v}_{r}\right)\right] d V+\int_{B_{k}} \rho\left(\mathbf{p} \times \mathbf{a}_{r}\right) d V\right\}
$$

and, for $r=4,5$, and 6 ,

$$
F_{r}^{*}=-m v_{u_{r}}^{s^{*}} \cdot s^{*}
$$

\subsubsection{Generalized Active Forces}

The force per unit volume, $\mathbf{F}^{P}$, acting on the generic particle $P$ of the system can be written as

$$
\mathbf{F}^{P}=\mathbf{F}_{e}^{P}+\mathbf{F}_{i}^{P}
$$

where $\mathbf{F}_{?}^{P}$ comes from forces external to the system, and $\mathbf{F}_{i}^{P}$ is from the internal forces acting on $P$. The generalized active force, $F_{r}$, for the system has the form 


$$
\begin{gathered}
F_{r}=\int_{B_{k}}\left[\left(\mathbf{F}_{e}^{p}+\mathbf{F}_{i}^{p}\right) \cdot \mathbf{v}_{u_{r}}\right] d V=\int_{B_{k}}\left[\left(\mathbf{F}_{e}^{p}+\mathbf{F}_{i}^{p}\right) \cdot\left(\mathbf{v}_{u_{r}}^{s^{*}}+\omega_{u_{r}} \times \mathbf{p}\right)\right] d V \\
=v_{u_{r}}^{s^{*}} \cdot\left(\int_{B_{k}} \mathbf{F}_{e}^{p} d V+\int_{B_{k}} \mathbf{F}_{i}^{p} d V\right)+\omega_{u_{r}} \cdot\left[\int_{B_{k}}\left(\mathbf{p} \times F_{e}^{p}\right) d V+\int_{B_{k}}\left(\mathbf{p} \times \mathrm{F}_{i}^{p}\right) d V\right]
\end{gathered}
$$

Now, if the internal force exerted on any particle $P$ of the system $S_{k}$ by another particle $Q$ of $\underline{S}_{\mathbf{R}}$ is assumed to act along the line connecting $P$ and $Q$, then

$$
\int_{B_{k}} F_{i}^{P} d V=0
$$

and

$$
\int_{B_{k}}\left(\mathbf{p} \times \mathbf{F}_{i}^{P}\right) d V=0
$$

In that case, the generalized active force can be written as

$$
F_{\mathrm{r}}=\mathbf{v}_{\mathrm{u}_{\mathrm{r}}}^{\mathrm{s}_{\mathrm{r}}^{*}} \cdot \mathbf{F}+\omega_{\mathrm{u}_{\mathrm{r}}} \cdot \mathbf{M}
$$

where $\mathbf{F}$ is the resultant external force on the system, and $\mathbf{M}$ is the sum of the moments of all the external forces on the system about the system mass center. (5) and (31) then give 


$$
F_{\mathrm{r}}=\omega_{\mathrm{u}_{\mathrm{r}}} \cdot \mathbf{M} \quad \text { for } \mathrm{r}=1,2,3
$$

and

$$
F_{\mathrm{r}}=\mathrm{v}_{\mathrm{u}_{\mathrm{r}}}^{\mathrm{s}^{*}} \cdot \mathrm{F} \quad \text { for } \mathrm{r}=4,5,6
$$

\subsubsection{Equations of Motion}

Recalling that Kane's equations have the form given in (1), we now combine (25) and (32) to obtain the equation of attitude motion as

$$
\tilde{\mathbf{I}} \cdot \alpha+\omega \times \tilde{\mathbf{I}} \cdot \omega+2 \int_{B_{k}} \rho\left[p \times\left(\omega \times v_{\mathrm{r}}\right)\right] d V+\int_{B_{k}} \rho\left(p \times a_{\mathrm{r}}\right) d V=M
$$

Similarly, the equation of translational motion comes from (26) and (33), and is

$$
\mathbf{m} \mathbf{a}^{s^{*}}=\mathbf{F}
$$

At this point, there are two main obstacles that prevent (34) and (35) from being useful as given above. First, the motion of fluid particles within $\mathbf{B}_{\mathbf{4}}$ was assumed known. This means that both the velocity and acceleration fields within $\mathbf{B}_{\mathbf{k}}$ are known as functions of time. In reality, none of these functions is known, but reasonable guesses can be made 
for the velocity field within $B$; that is, the velocity distribution inside $B_{4}$ for the instant $t=$ $t_{\mathbf{k}}$, when the constant mass system coincides with the variable mass system $S$. On the other hand, we have no handle over the velocity distribution of any particles outside $B$; and some such particles would normally be within $\mathbf{B}_{4}$ at instants of time different from $t_{k}$ The other problem is that we have no way of estimating the acceleration field - not even within B.

To circumvent these problems, two important measures are taken. One is that attempts are made to convert all accelerations that appear explicitly in (34) and (35) to time derivatives of velocity. If done properly, this would take care of the second problem above. The other measure is that ways are found to convert any volume integral that contains a velocity term, and is taken over the region $B_{4}$ to a volume integral over $B$, where velocities can be estimated. This measure would resolve the first problem. We now show how these measures can be applied to Eq. (35).

Substituting for $\mathrm{a}^{*}$ from (15), (35) becomes

$$
m\left[a^{o}+\alpha \times r^{*}+\omega \times\left(\omega \times r^{*}\right)+2 \omega \times v_{r}^{s^{*}}+a_{r}^{s^{*}}\right]=F
$$

It is convenient to express (36) as

$$
\mathrm{m}\left[\mathrm{a}^{o}+\alpha \times \mathbf{r}^{*}+\omega \times\left(\omega \times \mathbf{r}^{*}\right)\right]+2 \int_{B_{k}} \rho\left(\omega \times v_{r}\right) d V+\int_{B_{k}} \rho a_{r} d V=F
$$

We then re-write the last term on the left hand side of (37) as 


$$
\int_{B_{k}} \rho \mathbf{a}_{r} d V=\int_{B_{k}} \rho \frac{R_{\mathrm{d}} \mathbf{v}_{r}}{\mathrm{dt}} d V=\frac{R_{\mathrm{d}}}{\mathrm{dt}} \int_{B_{k}} \rho \mathbf{v}_{r} d V
$$

where the left superscript, $R$, indicates the reference frame in which the time derivative is taken. Next, we substitute (38) into (37) and evaluate the new expression at time $t_{k}$, when $\underline{S}_{\mathbf{R}}$ is identical with $S$, and obtain

$$
m\left[\mathbf{a}^{o}+\alpha \times \mathbf{r}^{*}+\omega \times\left(\omega \times \mathbf{r}^{*}\right)\right]_{t=t_{k}}+2 \int_{B} \rho\left(\omega \times v_{r}\right) d V+\left[\frac{R_{\mathrm{d}}}{\mathrm{dt}} \int_{B_{k}} \rho \mathbf{v}_{r} d V\right]_{t=t_{k}}=\mathbf{F}\left(t_{k}\right)
$$

Because the last term on the left hand side of (39) contains a time derivative outside the integral sign, the limit of the integral cannot be changed to B as was done for the previous term. We are thus faced with the problem of taking the integral of an expression containing a velocity term over a region in which the velocity field is not known - the first problem discussed above. Fortunately, this dilemma can be resolved by means of the Reynolds Transport Theorem, which gives in this case,

$$
\left[\frac{R_{\mathrm{d}}}{\mathrm{dt}} \int_{B_{k}} \rho \mathbf{v}_{r} d V\right]_{t=t_{k}}=\left[\frac{R_{\mathrm{d}}}{\mathrm{dt}} \int_{B_{k}} \rho \mathbf{v}_{r} d V+\int_{S} \rho \mathbf{v}_{\mathrm{r}}\left(\mathbf{v}_{r} \cdot \mathbf{n}\right) d S\right]_{t=t_{k}}
$$

where $S$ is the surface area of $B$, and $\mathbf{n}$ is a unit vector normal to $B$ and pointing outwards. Eqs. (39) and (40) thus give 


$$
\begin{gathered}
\mathrm{m}\left[\mathrm{a}^{o}+\alpha \times \mathrm{r}^{*}+\omega \times\left(\omega \times \mathrm{r}^{*}\right)\right]_{t=t_{k}}+2\left[\int_{B_{k}} \rho\left(\omega \times \mathrm{v}_{r}\right) d V\right]_{t=t_{k}} \\
+\left(\frac{R_{\mathrm{d}}}{\mathrm{dt}} \int_{C} \rho \mathbf{v}_{r} d V\right)_{t=t_{k}}+\left[\int_{S} \rho \mathbf{v}_{r}\left(\mathbf{v}_{r} \cdot \mathbf{n}\right) d S\right]_{t=t_{k}}=\mathbf{F}\left(t_{k}\right)
\end{gathered}
$$

where $m$ is the mass of $\underline{S}_{\mathbf{k}}$ at time $t_{k}$; that is, the mass of $S$ at time $t_{k}$. Because (41) has the same form for all values of $t_{k}$, the equation of translational motion for the full variable mass system is

$$
\begin{gathered}
m\left[a^{o}+\alpha \times r^{*}+\omega \times\left(\omega \times r^{*}\right)\right]+2 \int_{B_{k}} \rho\left(\omega \times v_{r}\right) d V \\
+\frac{R_{d}}{d t} \int_{B} \rho v_{r} d V+\int_{S} \rho v_{r}\left(v_{r} \cdot n\right) d S=F
\end{gathered}
$$

The equation of rotational motion for the variable mass system is obtained in a similar way from (34). First, we have that

$$
\int_{B_{k}} \rho\left(\mathbf{p} \times \mathbf{a}_{r}\right) d V=\int_{B_{k}} \rho \frac{{ }^{R} d}{d t}\left(\mathbf{p} \times \mathbf{v}_{r}\right) d V=\frac{{ }^{R} d}{d t} \int_{B_{k}} \rho\left(\mathbf{p} \times \mathbf{v}_{r}\right) d V
$$


Then we invoke Reynolds Transport Theorem once again to convert (43) to

$$
\left[\int_{B_{k}} \rho\left(\mathbf{p} \times \mathbf{a}_{r}\right) d V\right]_{t=t_{k}}=\left[\frac{R_{d}}{d t} \int_{B_{k}} \rho\left(\mathbf{p} \times \mathbf{v}_{r}\right) d V+\int_{S} \rho\left(\mathbf{p} \times \mathbf{v}_{r}\right)\left(\mathbf{v}_{r} \cdot \mathbf{n}\right) d S\right]_{t=t_{k}}
$$

Thus, the equation of rotational motion becomes [from (34) \& (44)]

$$
\begin{gathered}
\tilde{\mathbf{I}} \cdot \boldsymbol{\alpha}+\omega \times \tilde{\mathbf{I}}_{\mathrm{s}} \cdot \boldsymbol{\omega}+2 \int_{B_{k}} \rho\left[\mathrm{p} \times\left(\omega \times \mathrm{v}_{\mathrm{r}}\right)\right] d V \\
+\frac{{ }^{R}}{d r} \int_{B_{k}} \rho\left(\mathrm{p} \times \mathrm{v}_{\mathrm{r}}\right) d V+\int_{S} \rho\left(\mathrm{p} \times \mathrm{v}_{\mathrm{r}}\right)\left(\mathbf{v}_{\mathrm{r}} \cdot \mathbf{n}\right) d S \cong \mathbf{M}
\end{gathered}
$$

where the inertia dyadic that appears in (45) is that of matter that is within B at the instant under consideration.

The vector equations of motion, (42) and (45), obtained through the application of Kane's equations, are identical to those obtained by other authors [ Meirovitch (1970), Wang (1993)] using Newton-Euler formulation. 


\subsubsection{Another form of the Equation of Attitude Motion}

Another very useful form of the vector equation of rotational motion can be obtained as follows. First, we go back to the equation of attitude motion of a representative constant mass system as given in Eq. (34), and consider the expression

$$
\mathbf{A}=\frac{{ }^{R} d}{d t} \int_{B_{k}}[\mathbf{p} \times(\boldsymbol{\omega} \times \mathbf{p})] d m=\frac{{ }^{R} d}{d t} \int_{B_{k}} \rho[\mathbf{p} \times(\boldsymbol{\omega} \times \mathbf{p})] d V
$$

This expression can be expanded in the following way :

$$
\begin{gathered}
\mathbf{A}=\frac{{ }^{R} d}{d t} \int_{C_{k}}[\mathbf{p} \times(\boldsymbol{\omega} \times \mathbf{p})] d m=\int_{B_{k}} \frac{{ }^{R}}{d t}[\mathbf{p} \times(\boldsymbol{\omega} \times \mathbf{p})] d m \\
=\int_{B_{k}}\left[\mathbf{v}_{r} \times(\boldsymbol{\omega} \times \mathbf{p})\right] d m+\int_{B_{k}}[\mathbf{p} \times(\boldsymbol{\alpha} \times \mathbf{p})] d m+\int_{B_{k}}\left[\mathbf{p} \times\left(\boldsymbol{\omega} \times \mathbf{v}_{r}\right)\right] d m
\end{gathered}
$$

The last term on the right hand side of (47) can be added and subtracted from (47), then by using the following equality

$$
\left[\mathbf{v}_{r} \times(\omega \times \mathbf{p})\right]-\left[\mathbf{p} \times\left(\omega \times \mathbf{v}_{r}\right)\right]=\left[\omega \times\left(\mathbf{v}_{r} \times \mathbf{p}\right)\right]
$$

we have 


$$
\mathbf{A}=\int_{B_{k}}\left[\boldsymbol{\omega} \times\left(\mathbf{v}_{r} \times \mathbf{p}\right)\right] d m+\tilde{\mathbf{I}} \cdot \boldsymbol{\alpha}+2 \int_{B_{k}}\left[\mathbf{p} \times\left(\boldsymbol{\omega} \times \mathbf{v}_{r}\right)\right] d m
$$

On the other hand, $A$, as given in (47), can be evaluated at $t=t_{k}$ and expanded using Reynolds Transport Theorem to yield

$$
\begin{aligned}
& \left\{\mathbf{A}\left(t_{k}\right)=\frac{{ }^{R}}{d t} \int_{B}[\mathbf{p} \times(\boldsymbol{\omega} \times \mathbf{p})] d m+\int_{S} \rho[\mathbf{p} \times(\boldsymbol{\omega} \times \mathbf{p})]\left(\mathbf{v}_{r} \cdot \mathbf{n}\right) d S\right\}_{t=t} \\
& =\left\{\frac{{ }^{R}}{d t}(\tilde{\mathbf{I}} \cdot \boldsymbol{\omega})+\int_{S} \rho[\mathbf{p} \times(\boldsymbol{\omega} \times \mathbf{p})]\left(\mathbf{v}_{r} \cdot \mathbf{n}\right) d S\right\}_{t=t} \\
& =\left\{\left(\frac{{ }^{R} \tilde{\mathbf{I}}}{d t}\right) \cdot \omega+\tilde{\mathbf{I}} \cdot \boldsymbol{\alpha}+\int_{S} \rho[\mathbf{p} \times(\boldsymbol{\omega} \times \mathbf{p})]\left(\mathbf{v}_{r} \cdot \mathbf{n}\right) d S\right\}_{t=t^{\prime}} .
\end{aligned}
$$

We then have, from (49) and (50), that

$$
2\left[\int_{B_{k}{ }^{\prime}} \rho \mathrm{p} \times\left(\omega \times \mathrm{v}_{\mathrm{r}}\right) d V\right]_{t=t_{k}}=\left\{\left(\frac{{ }^{R} d \tilde{\mathrm{I}}}{d t}\right) \cdot \omega+\int_{S} \rho[\mathrm{p} \times(\omega \times \mathrm{p})]\left(\mathrm{v}_{\mathrm{r}} \cdot \mathbf{n}\right) d S+\int_{B_{k}} \rho\left[\omega \times\left(\mathrm{p} \times \mathrm{v}_{\mathrm{r}}\right)\right] d V\right\}
$$


Eqs. (34) and (51) lead to the following alternate form of the vector equation of attitude motion:

$$
\begin{gathered}
\tilde{\mathbf{I}} \cdot \boldsymbol{\alpha}+\boldsymbol{\omega} \times \tilde{\mathbf{I}} \cdot \boldsymbol{\omega}+\left(\frac{{ }^{R} d \tilde{\mathbf{I}}}{d t}\right) \cdot \boldsymbol{\omega}+\int_{S} \rho[\mathbf{p} \times(\boldsymbol{\omega} \times \mathbf{p})]\left(\mathbf{v}_{r} \cdot \mathbf{n}\right) d S+ \\
\int_{B} \rho\left[\boldsymbol{\omega} \times\left(\mathbf{p} \times \mathbf{v}_{r}\right)\right] d V+\frac{{ }^{R} d}{d t} \int_{B} \rho\left(\mathbf{p} \times \mathbf{v}_{r}\right) d V+\int_{S} \rho\left(\mathbf{p} \times \mathbf{v}_{r}\right)\left(\mathbf{v}_{r} \cdot \mathbf{n}\right) d S=\mathbf{M}
\end{gathered}
$$




\section{CHAPTER 3}

\section{EFFECTS OF MASS VARIATION}

The equations of motion derived in chapter 2 [see (2.42), (2.45) and (2.52)] are quite complex; and very little progress can be made with these equations without some simplifying assumptions to reduce their complexity. In this chapter, these equations are reexamined. The significance of each of the terms is evaluated, and, the circumstances under which some of these terms can be dropped are explored. The goal is to arrive at a set of equations that capture the salient features of the system under study, and that are nevertheless simple enough to render further analysis tractable.

\subsection{Translational Motion}

The vector equation of translational motion [see (2.42)] is reproduced here as

$$
\begin{gathered}
m\left[\mathbf{a}^{o}+\alpha \times \mathbf{r}^{*}+\omega \times\left(\omega \times \mathbf{r}^{*}\right)\right]+2 \int_{B_{k}} \rho\left(\omega \times v_{r}\right) d V \\
+\frac{R_{d}}{d t} \int_{B} \rho v_{r} d V+\int_{S} \rho v_{r}\left(v_{r} \cdot \mathbf{n}\right) d S=\mathbf{F}
\end{gathered}
$$


This equation can be re-written as

$$
m\left[a^{0}+\alpha \times r^{*}+\omega \times\left(\omega \times r^{*}\right)\right]=F+F_{C}+F_{L}+F_{T}
$$

where

$$
\begin{gathered}
F_{C}=-2 \int_{B_{k}} \rho\left(\omega \times v_{r}\right) d V \\
F_{L}=-\frac{{ }^{R} d}{d t} \int_{B} \rho v_{r} d V
\end{gathered}
$$

and

$$
\mathbf{F}_{T}=-\int_{S} \rho \mathbf{v}_{\mathbf{r}}\left(\mathbf{v}_{r} \cdot \mathbf{n}\right) d S
$$

If $\mathbf{v}_{r}$ is identically zero everywhere, then $\mathbf{F}_{c}=\mathbf{F}_{L}=\mathbf{F}_{T}=0$, and we recover the equations of translational motion for a rigid body. Thus, mass variation appears to augment the "external forces" on an "equivalent rigid body" by three terms. $F_{C}$ is often referred to in the literature as the Coriolis force, since it derives from the Coriolis component of the acceleration. $\mathbf{F}_{\mathrm{L}}$ is the rate at which the system's linear momentum relative to $\mathrm{B}$ decreases with time because of particle motion inside $B$. $\mathbf{F}_{\mathrm{T}}$ represents the rate at which relative linear momentum is lost across the boundary $B$, and is often referred to as the thrust vector in rocket applications.

If those particles of the system that can move relative to $B$ are allowed to move within $B$ but do not cross the boundary $B$, then $\mathbf{F}_{\mathrm{T}}$ becomes zero. $\mathbf{F}_{\mathrm{T}}$ would be non-zero but negligible if either a very small percentage of the system's particles is allowed to cross 
$\mathrm{B}$, or those particles that cross B do so at very slow rate. In other words, the thrust vector can be neglected whenever the amount of matter that is lost or gained per unit time is small. What matters is the rate, not the total amount of matter lost or gained.

Note that we can use Reynold's transport theorem to expand the Coriolis force into

$$
\begin{gathered}
F_{c}=-2 \omega \times \int_{B_{k}} \rho \mathbf{v}_{r} d V=-2 \omega \times \frac{R}{d t} \int_{B_{k}} \rho \mathbf{r} d V \\
=-2 \omega \times\left(\frac{{ }^{R}}{d t} \int_{B} \rho \mathbf{r} d V+\int_{S} \rho \mathbf{r}\left(\mathbf{v}_{r} \cdot n\right) d S\right)=\mathrm{F}_{c_{1}}+\mathrm{F}_{c_{2}}
\end{gathered}
$$

where

$$
\mathrm{F}_{c_{1}}=-2 \omega \times \frac{{ }^{R} d}{d t} \int_{B} \rho \mathrm{r} d V
$$

and

$$
F_{c_{2}}=-2 \omega \times \int_{s} \rho r\left(v_{r} \cdot n\right) d S
$$

There are situations where $\mathbf{v}_{\mathrm{r}}$ can be considered negligible inside the boundary $B$ but not at an exit from B. An example is an inflated balloon with a hole. Gas motion inside the balloon is hardly noticeable while gas exit velocity at the hole is substantial. In cases like this, $\mathbf{F}_{L}$ and the first term of $\mathbf{F}_{C}\left(\mathbf{F}_{C 1}\right)$ are negligible, but $\mathbf{F}_{C 2}$ as well as the thrust vector survive. As a matter of fact, this is not an unreasonable assumption for practical systems such as rockets, and can provide a way of rendering the equations of such systems tractable, since the details of internal gas flow can be neglected. Even if $\mathbf{v}_{\mathrm{r}}$ is not negligible 
within B, but the particles within B can attain some type of steady state in their motion relative to $B$, then $F_{L}$ and $F_{C l}$ are once more negligible. After ignition, rocket systems quickly attain an approximate steady state, and so, $\mathbf{F}_{L}$ and $\mathbf{F}_{C l}$ can be ignored for such systems.

In summary, the vector equation of translational motion for variable mass systems can, in many cases be reduced to

$$
m\left[\mathbf{a}^{0}+\alpha \times \mathbf{r}^{*}+\omega \times\left(\omega \times r^{*}\right)\right]=F+F_{T}+F_{C_{2}}
$$

where $F$ is the external force, $F_{T}$ is the thrust vector, and $F_{C 2}$ is part of the Coriolis force and is given by (8). $F_{C}$ and $F_{T}$ can be dropped completely only when the rate of mass loss is negligible.

\subsection{Rotational Motion}

Two forms of the vector equation of attitude motion were developed in chapter 2 [(2.45) and (2.52)], and are reproduced below.

$$
\begin{gathered}
\tilde{\mathbf{I}} \cdot \boldsymbol{\alpha}+\boldsymbol{\omega} \times \tilde{\mathbf{I}} \cdot \boldsymbol{\omega}+\mathbf{2} \int_{B_{k}} \rho\left[\mathbf{p} \times\left(\boldsymbol{\omega} \times \mathbf{v}_{\mathrm{r}}\right)\right] d V \\
+\frac{{ }^{R} d}{d t} \int_{B_{k}} \rho\left(\mathbf{p} \times \mathbf{v}_{\mathrm{r}}\right) d V+\int_{S} \rho\left(\mathbf{p} \times \mathbf{v}_{\mathrm{r}}\right)\left(\mathbf{v}_{\mathrm{r}} \cdot \mathbf{n}\right) d S=\mathbf{M}
\end{gathered}
$$




$$
\begin{gathered}
\tilde{\mathbf{I}} \cdot \boldsymbol{\alpha}+\boldsymbol{\omega} \times \tilde{\mathbf{I}} \cdot \boldsymbol{\omega}+\left(\frac{{ }^{R} d \tilde{\mathbf{I}}}{d t}\right) \cdot \omega+\int_{S} \rho[\mathbf{p} \times(\boldsymbol{\omega} \times \mathbf{p})]\left(\mathbf{v}_{r} \cdot \mathbf{n}\right) d S+ \\
\int_{B} \rho\left[\omega \times\left(\mathbf{p} \times \mathbf{v}_{r}\right)\right] d V+\frac{{ }^{R}}{d t} \int_{B} \rho\left(\mathbf{p} \times v_{r}\right) d V+\int_{S} \rho\left(\mathbf{p} \times v_{r}\right)\left(\mathbf{v}_{r} \cdot \mathbf{n}\right) d S=\mathbf{M}
\end{gathered}
$$

Both of these equations reduce to rigid body rotational equations if $v_{z}=0$ within $B$ as well as on B. Thus (10) can be written as

$$
\tilde{\mathbf{I}} \cdot \boldsymbol{\alpha}+\boldsymbol{\omega} \times \tilde{\mathbf{I}} \cdot \boldsymbol{\omega}=\mathbf{M}+\mathbf{M}_{\mathrm{C}}+\mathbf{M}_{\mathrm{H}}+\mathbf{M}_{\mathrm{T}}
$$

where

$$
\mathbf{M}_{C}=-2 \int_{B_{k}} \rho\left[\mathrm{p} \times\left(\omega \times \mathrm{v}_{r}\right)\right] d V
$$

is the Coriolis moment,

$$
\mathbf{M}_{H}=-\frac{R^{d}}{d t} \int_{B} \rho\left(\mathbf{p} \times \mathbf{v}_{r}\right) d V
$$

represents the rate of decrease of the system's relative angular momentum inside B, and 


$$
\mathbf{M}_{T}=-\int_{S} \rho\left(\mathbf{p} \times \mathbf{v}_{r}\right)\left(\mathbf{v}_{r} \cdot \mathbf{n}\right) d S
$$

is the rate of loss of relative angular momentum across the boundary, and is also equal to the moment of the thrust vector about the system mass center.

In those situations where it is reasonable to assume that the motion of the fluid phase relative to the solid phase has axial symmetry, and when there is no whirling motion, we have that

$$
\int_{B_{k}} \rho\left(p \times v_{r}\right) d V=\int_{B} \rho\left(p \times v_{r}\right) d V \equiv 0
$$

This is so because, for every particle $P$ of the system with position vector $\mathbf{p}$ and relative velocity $v_{r}$ there exists another particle $P^{\prime}$ of position vector $p^{\prime}$ and relative velocity $v_{r}^{\prime}$ such that the vectors $p \times v_{r}$ and $p^{\prime} \times v_{r}^{\prime}$ have the same magnitude but opposite directions. And, since axisymmetry also implies that the immediate neighborhoods of $\mathrm{P}$ and $\mathrm{P}$ ' have the same mass density, Eq. (3.16) follows. This equation immediately leads to

$$
\mathbf{M}_{H}=\mathbf{M}_{T}=0
$$

We arrive at the same conclusion for systems where $v_{r}$ can be considered negligible inside the boundary $B$ but not at an exit from $B$, such as rockets. $M_{H}$ is also negligible for steady state relative motion of the fluid particles.

From (10) and (11) the Coriolis moment can be expanded to 


$$
\mathbf{M}_{C}=-\left[\left(\frac{{ }^{R} d \tilde{\mathbf{I}}}{d t}\right) \cdot \omega+\int_{B} \rho[\mathbf{p} \times(\omega \times \mathbf{p})]\left(\mathbf{v}_{r} \cdot \mathbf{n}\right) d S+\int_{B_{k}} \rho\left[\omega \times\left(\mathbf{p} \times \mathbf{v}_{r}\right)\right] d V\right]
$$

The last term on the right hand side of (18) vanishes for axisymmetric motion as well as for negligible internal flow. The second term on the right hand side of (18) is often referred to as the jet damping moment, because it has been shown [see Dryer (1963)] to have attenuating effects on the angular rates in some types of rocket systems. To evaluate this surface integral, it is necessary to know the geometric shape of those parts of the boundary where particles are allowed to exit or enter the system, as well as the velocity profile at these locations. This moment is thus very much dependent on the system's geometry. In the case of rockets, for example, the longitudinal dimension of the combustion chamber (for solid rockets) as well as the exit nozzle radius have much to do with the impact of this term on the system's attitude motion.

The first term on the right hand side of (18) captures the contribution of inertia variation. We thus conclude that jet damping moment and the moment due to the changes in inertia properties have dominant effects on the attitude dynamics of the system. The relative importance and the interaction between these two moments essentially determine the character of the attitude dynamics of variable mass systems. There is advantage in using the reduced form of (11) for the study of rotational motion. If the assumptions of symmetric and/or negligible internal motion are made, the most "troublesome" terms will have dropped out. The only term in this equation that would contain $\mathbf{v}_{\mathbf{r}}$ is a surface integral over B. $v_{\mathbf{T}} \cdot \mathbf{n}$ is zero everywhere on the surface of $B$ except at those places where fluid particles can enter or leave the region delimited by B (the nozzle exit plane in the case of 
rockets). In general, $\mathbf{v}_{\mathbf{r}} \cdot \mathbf{n}$ can be approximated relatively well at these locations; hence the surface integral can in fact be evaluated in closed form.

In the study of the dynamic behavior of variable mass mechanical systems, the most important forces appear to be the thrust vector and the Coriolis force. The jet damping moment and the moment due to inertia variation are the dominant moments. These quantities should be included in any dynamic studies of variable mass systems to ensure that meaningful predictions can be made from the analysis. 


\section{CHAPTER 4}

\section{ATTITUDE MOTIONS OF A VARIABLE MASS CYLINDER}

\subsection{Introduction}

In this chapter, we begin to narrow the scope of our study to an important and useful class of variable mass systems - space rockets. We start this process with an indepth study of the attitude motions of a variable mass system that is initially a solid right circular cylinder, which loses its mass continuously through combustion as it moves around in space. The cylinder problem is a very important and useful problem for several reasons. First, it models rocket-type systems in a simple enough way that the equations of motion become relatively tractable. Generally, space vehicles are designed to be more or less axisymmetric is shape, and, a cylinder is actually a good - albeit rough approximation for such systems. Hence, a thorough study of the variable mass cylinder problem is a useful exercise in that it provides a means of performing tractable analytical studies of rocket-type systems, and can lead to great insight into the dynamic behavior of this class of variable mass systems. 
The idea of using a cylinder to study the attitude behavior of variable mass systems was originated by Eke and Wang (1995). In their work, they solved completely in closed form, the equations of motion of a variable mass cylinder for several burn scenarios. They then extracted useful qualitative as well as quantitative information about the attitude behavior of the cylinder from the analytical expressions of the solutions of the equations of motion. The approach here is different. The strategy here is to develop an analytical method that yields a wealth of fundamental information about the behavior of the system without actually solving the equations of motion. The main motivation for this approach is that such a strategy can be applied to more complicated models of variable mass systems for which closed form solutions cannot be found.

\subsection{Attitude Equations for the Cylinder}

Consider a variable mass system of the rocket type that is initially a right circular cylinder as shown in dotted lines in Fig. 1. This system is given an initial angular velocity $\omega_{b}$, and is then allowed to move freely in a torque-free environment as it loses its mass continuously through combustion or similar processes. The intent is to perform qualitative and quantitative studies of the attitude motions of such a system. To begin, we define as control region, the space enclosed in dotted lines in Fig. 1. Matter within this region at any given instant is considered part of the system at that instant.

The attitude motions of such a system are governed by Eq. (2.52), which can be simplified, as discussed in chapter 3 , by making the following assumptions: (a) the burning process proceeds in such a way that the solid unburned part of the cylinder remains symmetrical about the original cylinder axis at all times; (b) the fluid products of combustion move in an axisymmetric manner relative to the solid unburned part of the 
cylinder, (c) whirling motion of the products of combustion relative to the solid portion of the system is negligible. With these assumptions, (2.52) reduces to

$$
\mathbf{M}=\tilde{\mathbf{I}} \cdot \boldsymbol{\alpha}+\boldsymbol{\omega} \times \tilde{\mathbf{I}} \cdot \boldsymbol{\omega}+\left(\frac{{ }^{B} d \tilde{\mathbf{I}}}{d t}\right) \cdot \boldsymbol{\omega}+\int_{B} \rho[\mathbf{p} \times(\boldsymbol{\omega} \times \mathbf{p})]\left(\mathbf{v}_{r} \cdot \mathbf{n}\right) d S
$$

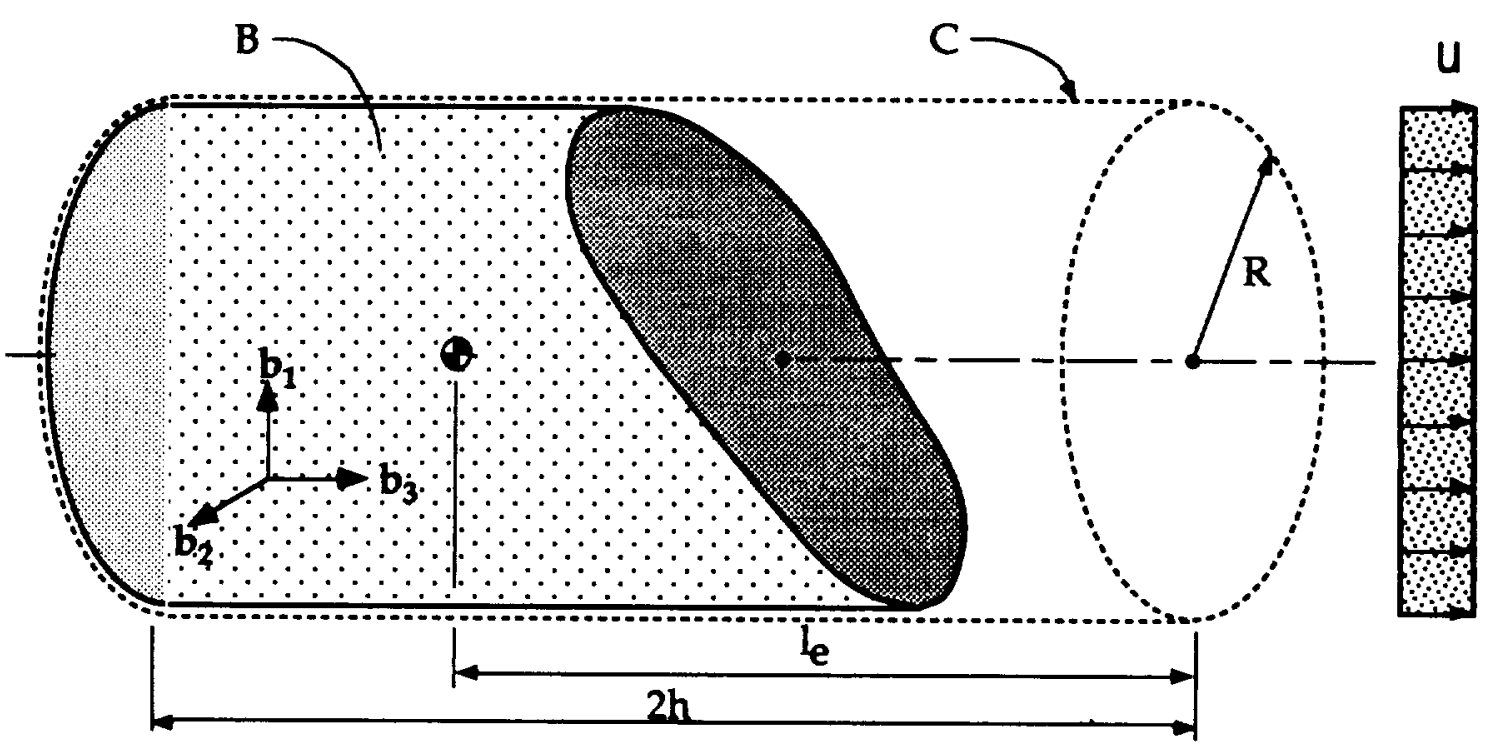

Fig. 4.1 Variable Mass Cylinder

The axisymmetry assumption allows us to express the instantaneous central inertia dyadic of the system as

$$
\tilde{\mathbf{I}}=I\left(\mathbf{b}_{1} \mathbf{b}_{1}+\mathbf{b}_{2} \mathbf{b}_{2}\right)+I_{z} \mathbf{b}_{3} \mathbf{b}_{3}
$$


where $\mathbf{b}_{1}, \mathbf{b}_{2}, \mathbf{b}_{3}$ are unit vectors fixed in B and directed as shown in Fig. 1 ; and $I$ and $I_{z}$ are respectively the transverse and axial central inertia scalars of the system. If the inertial angular velocity of $B$ at some general instant of time is taken to be

$$
\omega=\omega_{x} b_{1}+\omega_{y} b_{2}+\omega_{2} b_{3}
$$

then, the angular acceleration is

$$
\alpha=\dot{\omega}_{x} b_{1}+\dot{\omega}_{y} b_{2}+\dot{\omega}_{2} b_{3}
$$

and the various terms of Eq. (1) become

$$
\begin{aligned}
& \tilde{\mathbf{I}} \cdot \boldsymbol{\alpha}=I\left(\dot{\omega}_{2} \mathbf{b}_{1}+\dot{\omega}_{1} \mathbf{b}_{2}\right)+I_{2} \dot{\omega}_{2} \mathbf{b}_{3} \\
& \omega \times \tilde{\mathbf{I}} \cdot \omega=\left(I_{z}-I\right) \omega_{2}\left(\omega_{1} \mathbf{b}_{1}-\omega_{\lambda} \mathbf{b}_{2}\right) \\
& \left(\frac{{ }^{B}}{d t} \tilde{\mathbf{I}}\right) \cdot \boldsymbol{\omega}=I\left(\omega_{\lambda} \mathbf{b}_{1}+\omega_{j} \mathbf{b}_{2}\right)+\dot{I}_{2} \omega_{z} \mathbf{b}_{3}
\end{aligned}
$$

In order to evaluate the last term of (1), an assumption must be made conceming the velocity field of the fluid particles of the system at the surface of the control region. Here, we assume that the vector $v_{r}$ is zero everywhere on $B$ except at one of the ends of the cylinder-the right end, say. And there,

$$
\mathbf{v}_{r} \cdot \mathbf{n}=u(r)
$$

because of our axisymmetry assumption. If it is further assumed that 


$$
u(r)=u=\text { constant }
$$

then

$$
\int_{B} \rho\left[\mathrm{p} \times(\omega \times \mathrm{p})\left(\mathrm{v}_{r} \cdot \mathbf{n}\right)\right] d S=-\dot{m}\left[\left(\ell^{2}+\frac{1}{4} R^{2}\right)\left(\omega_{1} \mathrm{~b}_{1}+\omega_{1} \mathrm{~b}_{2}\right)+\frac{1}{2} R^{2} \omega_{2} \mathrm{~b}_{3}\right]
$$

where $m$ is the instantaneous mass of the system, and $l(t)$ is the distance of the mass center from the right face of the shell, B. By substituting (5), (6), (7) and (10) into (1) and setting $\mathbf{M}$ to zero, we obtain the scalar equations that describe the torque-free attitude motion of the variable mass cylinder, and they are

$$
\begin{gathered}
I \dot{\omega}_{x}+\left(I_{z}-I\right) \omega_{y} \omega_{z}+\left\{\dot{I}-\dot{m}\left(\ell^{2}+R^{2 / 4}\right)\right\} \omega_{x}=0 \\
I \dot{\omega}_{y}-\left(I_{z}-I\right) \omega_{x} \omega_{z}+\left\{\dot{I}-\dot{m}\left(\ell^{2}+R^{2 / 4}\right)\right\} \omega_{y}=0 \\
I_{z} \dot{\omega}_{z}+\left\{\dot{I}_{z}-\dot{m} R^{2 / 2}\right\} \omega_{z}=0
\end{gathered}
$$

\subsection{Attitude Stability}

Multiplying (11) by $\omega_{x},(12)$ by $\omega_{y}$ and adding the two resulting equations leads to

$$
\frac{1}{2} \mathrm{I} \frac{\mathrm{d}}{\mathrm{dt}}\left(\omega_{x}^{2}+\omega_{y}^{2}\right)+\left[\dot{\mathrm{I}}-\dot{\mathrm{m}}\left(\ell^{2}+\mathrm{R}^{2} / 4\right)\right]\left(\omega_{x}^{2}+\omega_{y}^{2}\right)=0
$$


We now let

$$
\begin{gathered}
\theta=\dot{\mathrm{I}}_{z}-\dot{\mathrm{m}} \mathrm{R}^{2} / 2 \\
\phi=\dot{\mathrm{I}}-\dot{\mathrm{m}}\left(\ell^{2}+\mathrm{R}^{2 / 4}\right)
\end{gathered}
$$

and

$$
\omega_{t}=\omega_{x}^{2}+\omega_{y}^{2}
$$

(13) and (14) can then be written more compactly as

$$
I_{z} \frac{d \omega_{z}}{d t}+\theta \omega_{z}=0
$$

and

$$
\mathrm{I} \frac{\mathrm{d} \omega_{t}}{\mathrm{dt}}+2 \phi \omega_{t}=0
$$

If the functions $\theta(t)$ and $\phi(t)$ are known, (18) and (19) can serve as basis for the determination of the essential features of the rotational motion of the variable mass cylinder. We note that these equations are uncoupled, so that the spin rate has no effect on the transverse angular rates. We also note that if $\theta(t)=0$, then the spin rate remains constant at its initial value throughout. Similarly, if $\phi(t)=0$, the magnitude of the transverse angular velocity does not change during the burn.

Equations (18) and (19) lead to

$$
\omega_{z}=\omega_{z} 0 \exp \left[-\int \frac{\theta(t)}{I_{z}(t)} d t\right]
$$


$\omega_{t}=\omega_{t 0} \exp \left[-2 \int \frac{\phi(t)}{\mathrm{I}(\mathrm{t})} \mathrm{dt}\right]$

or

$$
\omega_{x y}=\omega_{x y 0} \exp \left[-\int \frac{\phi(t)}{\mathrm{I}(t)} d t\right]
$$

where $\omega_{x y}=\sqrt{\omega_{t}}$ is the magnitude of the transverse angular velocity, and the subscript 0 indicates initial value; that is, value at ignition. We shall use the subscript $\infty$ to indicate values at burnout.

Looking back at (15) and (16), we observe that each of the functions $\theta$ and $\phi$ is made up of two parts. For example,

$$
\phi=\phi_{1}+\phi_{2}
$$

where

$$
\phi_{1}=\dot{I}
$$

and

$$
\phi_{2}=-\dot{\mathrm{m}}\left(\ell^{2}+\mathrm{R}^{2} / 4\right)
$$

Because we are concerned here with a situation where mass is being lost, both $\dot{\mathbf{I}}$ and $\dot{\mathrm{m}}$ are negative. Hence, $\phi_{1}$ remains negative throughout the burn while $\phi_{2}$ is positive throughout. Thus, $\phi$ can be positive, negative or zero. $\phi_{1}$ is contributed by the change in 
system transverse inertia, and because it is always negative, it will tend to cause $\omega_{x y}$ to diverge [see (22)]. On the other hand, $\phi_{2}$ is, in this case, the so-called jet damping term, and it does in fact attenuate $\omega_{x y}$ by virtue of the fact that it is always positive. Although $\phi_{1}$ is determined by the burn geometry of the cylinder, $\phi_{2}$ depends on the size of the cylinder as well as the velocity distribution across its exit plane. All the statements made above about $\phi(t)$ apply equally to $\theta(t)$ since (15) and (16) have the same form, and (20) is similar to (22).

It is possible to extract important qualitative information about the attitude motions of the system from a detailed examination of the functions $\phi(t)$ and $\theta(t)$. From (20) and (22), it is clear that for $\theta(t)$ and $\phi(t)>0$, both $\omega_{z}$ and $\omega_{x y}$ approach zero from any initial conditions. On the other hand, for $\theta(t)$ and $\phi(t)<0$, both $\omega_{z}$ and $\omega_{x y}$ diverge. Profound changes in angular velocity can occur when variations are made in $\theta(t)$ and $\phi(t)$.

To make further progress with our study of the attitude behavior of the variable mass cylinder, we will now proceed with a close study of the functions $\theta(t)$ and $\phi(t)$. These functions require that the system mass, inertia scalars and some geometric properties be known as functions of time; and these parameters can be determined if the burn scenario is known. We will therefore consider, as did Eke and Wang(1995), four burn scenarios : the uniform burn, the end burn, the radial or centrifugal burn, and the centripetal or antiradial burn. In uniform burn, the assumption is that the cylinder is ignited simultaneously everywhere inside it at time $t_{0}$. It then burns in the same uniform manner everywhere, so that the external dimensions remain the same throughout, but the density of matter within the cylinder decreases uniformly in the same manner everywhere within the cylinder. The density is thus the same function of time everywhere inside the cylinder, and the products of combustion are expelled at one end of the cylinder. An end burning cylinder burns from one of its ends to the other, in such a way that the intermediate shape of the system is always a cylinder of the same radius but decreasing length. Once more, the products of combustion are expelled from one of the ends - the burning end. In radial burn, the axis of 
the cylinder is ignited at time $t_{0}$, and the system then burns radially outwards in such a way that the intermediate shape is a cylindrical pipe. Counter-radial or centripetal burn is the reverse of the radial burn. The cylinder is ignited at its periphery (but not the ends) and burns radially inwards, with the intermediate shape being a cylinder of constant length but of decreasing radius.

\subsubsection{The Uniform Burning Cylinder}

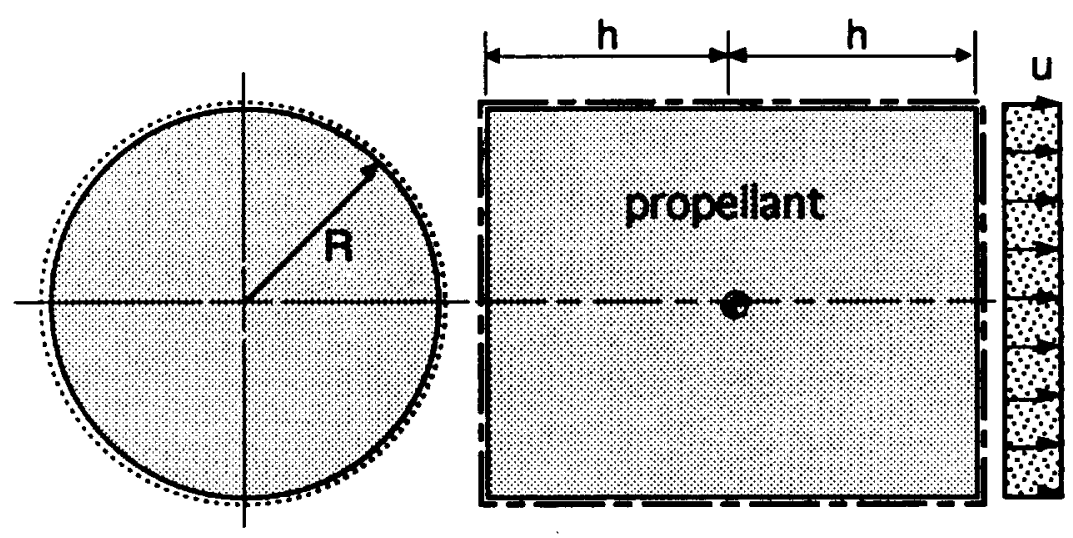

Fig. $\quad 4.2$ Cylinder in Uniform Burn

We now consider the case of a cylinder in uniform burn with uniform velocity profile at the exit plane, as shown in Fig. 2 above. With the dimensions shown in the figure, the inertia scalars are, in this case,

$$
I=m\left(R^{2 / 4}+h^{2 / 3}\right)
$$

and 


$$
I_{2}=m R^{2 / 2}
$$

Hence

$$
\dot{I}=\dot{m}\left(R^{2 / 4}+h^{2 / 3}\right)
$$

and

From (15) and (29), we have that

$$
\dot{\mathrm{I}}_{2}=\dot{\mathrm{m}} R^{2 / 2}
$$

$$
\theta(\mathrm{t})=0
$$

at all times, and so, the spin rate, $\omega_{z}$, will remain constant at its initial value.

From (16) and (28), and recalling that $\ell$ in (16) is the same as $h$ in Fig. 2 for this case,

$$
\phi(t)=-\frac{2}{3} \dot{m} h^{2}>0
$$

Because $\phi(t)>0$ at all times during the burn, the magnitude of the transverse angular velocity, $\omega_{\mathrm{xy}}$, decreases exponentially to zero.

\subsubsection{The End Burning Cylinder}

Fig. 3 shows the dimensions and intermediate shape of a cylinder in end burn. The auxiliary spatial coordinate $z$ is employed to characterize unburned propellant inertia properties, including mass, moments of inertia and the location of center of mass. The mass and inertia properties and their time derivatives are 


$$
\begin{gathered}
m=2 \rho \pi R^{2} z \\
\dot{m}=2 \rho \pi R^{2} \dot{z} \\
I=m\left(R^{2 / 4}+z^{2 / 3}\right) \\
\dot{I}=\dot{m}\left(R^{2 / 4}+z^{2}\right) \\
I_{z}=m R^{2 / 2} \\
\dot{I}_{z}=\dot{m} R^{2 / 2}
\end{gathered}
$$

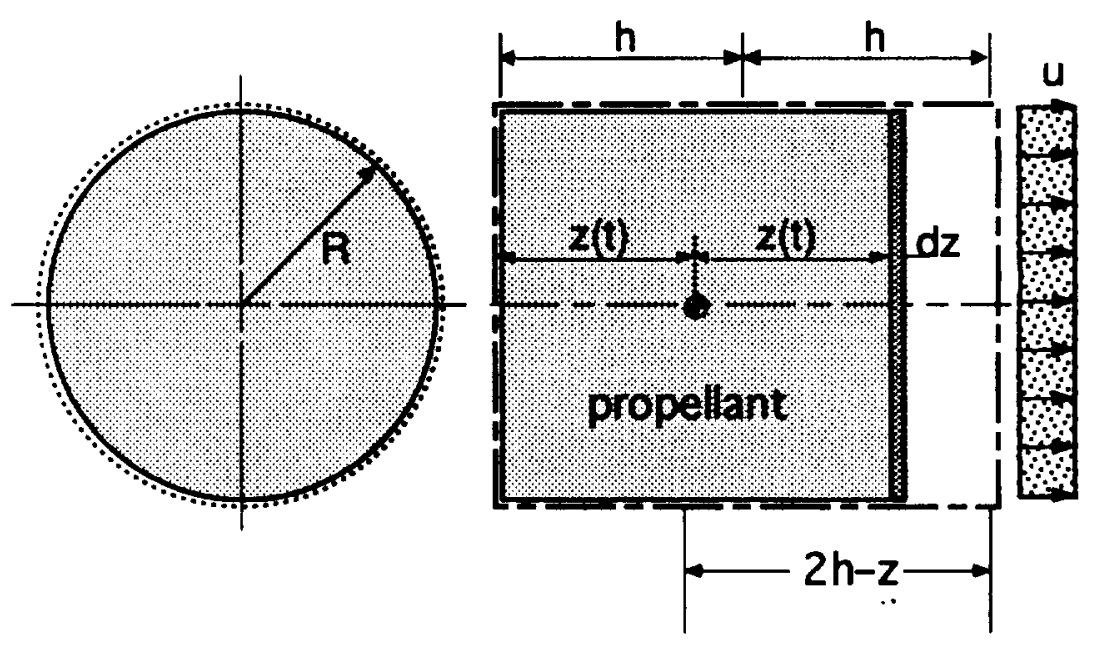

Fig. $\quad 4.3$ Cylinder in End Burn

Furthermore, 


$$
\ell=(2 \mathrm{~h}-2 z)+z=2 \mathrm{~h}-z
$$

so, from (16) and (38),

$$
\begin{gathered}
\phi(t)=\dot{m}\left(R^{2 / 4}+z^{2}\right)-\dot{m}\left[(2 h-z)^{2}+R^{2 / 4}\right] \\
=\dot{m} h^{2}[4(z / h)-4]
\end{gathered}
$$

At ignition $t=t_{0}$ and $z=h$, so that

$$
\phi_{0}=0
$$

At burnout $\mathrm{t}=\mathrm{t}_{\infty}$ and $\mathrm{z}=0$; hence

$$
\phi_{\infty}=-4 \dot{\mathrm{m}} \mathrm{h}^{2}>0
$$

From the mass continuity equation, we also have

$$
\dot{\mathrm{m}}=-\rho \pi \mathrm{R}^{2} \mathrm{u}<0
$$

Based on the assumption of uniform velocity profile at the exit plane, we conclude that $\dot{m}$ is constant during the time interval $\left[t_{0}, t_{\infty}\right]$. We also know that $\dot{z}$ is negative in this interval. From (39), we have that

$$
\mathrm{d} \phi / \mathrm{dt}=4 \mathrm{~h} \dot{\mathrm{m}} \dot{\mathrm{z}}>0
$$


between ignition and burnout. Thus, $\phi(t)$ increases monotonically from 0 to the positive value given in (41). We have a situation where $\phi(t)$ has the form shown in Fig. 4.

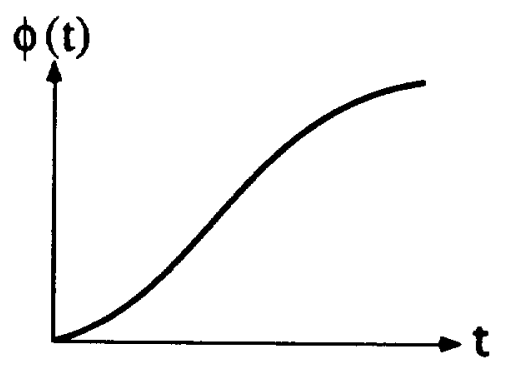

Fig. 4.4 The shape of $\phi(t)$ for End Burn

We conclude from (40), (41) and (43) that the transverse angular rate, $\omega_{x y}$, stays approximately constant during the initial phase of the burn, then decreases exponentially to zero.

As for the spin rate, (15) and (37) indicate that $\theta(t)=0$ for all $t$, so that the spin rate is again constant.

Having established that the transverse angular velocity of an end burning cylinder is always damped, our next task is to examine the effect of the cylinder's initial geometric configuration on the lateral response. To accomplish this task, let us define

$$
\Psi=\phi(t) / I(t)
$$

It is clear from (22) that this factor actually governs the speed with which the transverse angular speed approaches zero from any initial value. The larger the magnitude of $\Psi$, the higher the rate of decay of lateral motion.

For end burn, 


$$
\Psi=\frac{\phi}{I}=\frac{\dot{\mathrm{m}}}{\mathrm{m}} \frac{\left(4 \mathrm{hz}-4 \mathrm{~h}^{2}\right)}{\left(\mathrm{R}^{2 / 4}+\mathrm{z}^{2 / 3}\right)}
$$

By virtue of (32) and (42), (45) can be rewritten as

$$
\Psi=\frac{2 u(1-z / h)}{z\left[1 / 4(R / h)^{2}+1 / 3(z / h)^{2}\right]}
$$

To compare the behavior of cylinders of the same length but different initial external radii, we allow $R$ to vary while keeping $z, h$ and $u$ invariant. A close inspection of (46) indicates that increasing $R / h$ results in smaller values for $\Psi$. Hence, the transverse angular velocity magnitude, $\omega_{x y}$, converges more slowly as the ratio $R / h$ is increased. In other words, the transverse motion of prolate cylinders in end burn is highly damped while that of oblate cylinder is only very slightly damped. In the limiting case, as $R / h \rightarrow \infty, \Psi=0$, the motion is totally undamped as in the torque-free motion of a rigid cylinder. We conclude then that the amplitude of lateral oscillation of a variable mass cylinder in end burn can be influenced by the choice of its initial shape. Fig. 5 is obtained by numerical integration of (22) and demonstrates all the above properties.

\subsubsection{Radial Burn}

We recall that in radial or centrifugal burn, the cylinder starts burning on its axis, and the burn propagates radially outwards with time. The intermediate configuration of unburned propellant is a hollow cylinder, as shown in Fig. 6, and the mass and inertia properties in terms of auxiliary spatial coordinate $r$ have the form 


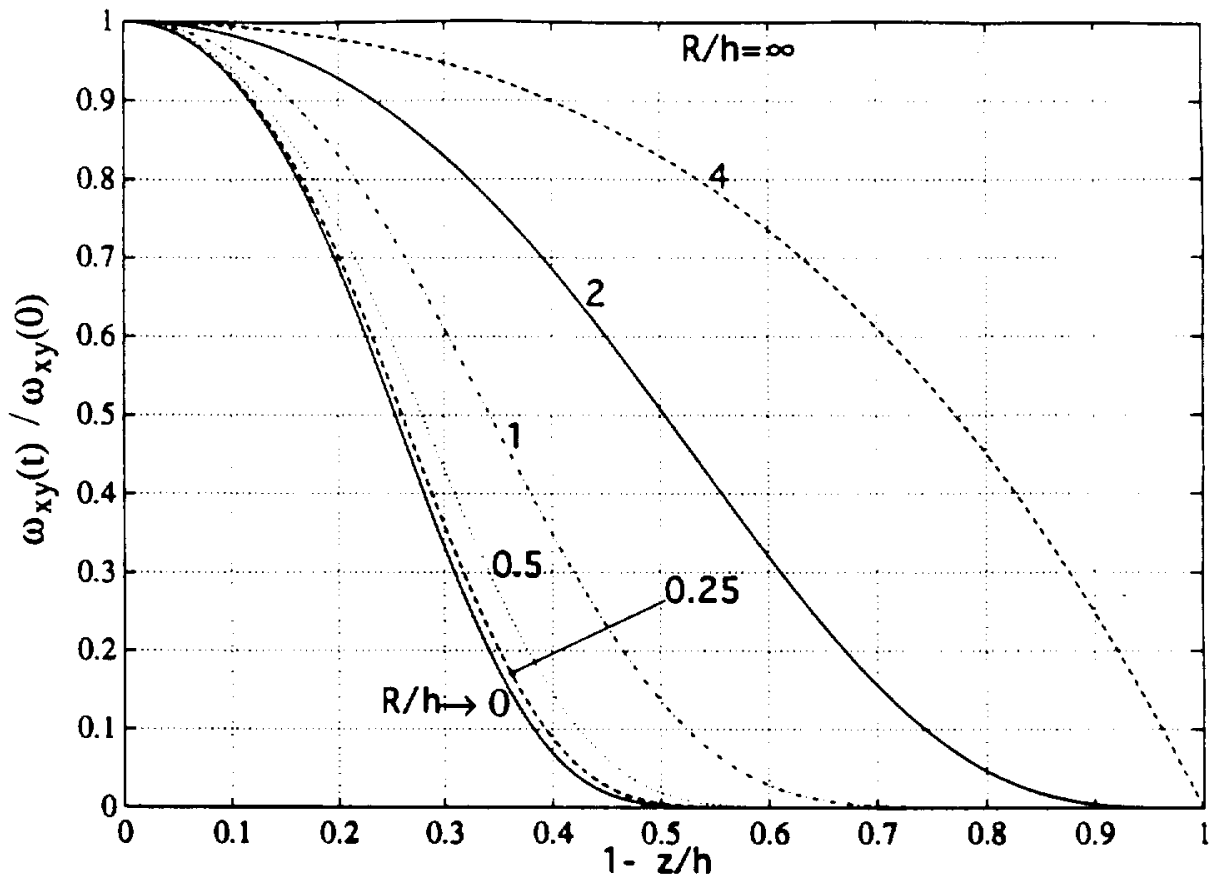

Fig. 4.5 Transverse Angular Rate for End Burn

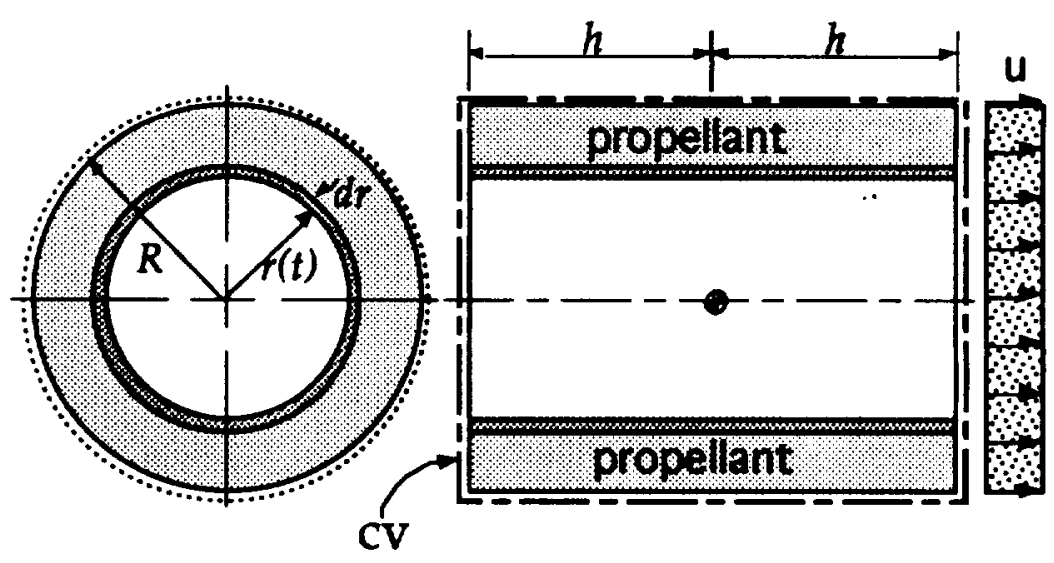

Fig. 4.6 Cylinder in Radial Burn 


$$
\begin{gathered}
m=2 \rho h \pi\left(R^{2}-r^{2}\right) \\
I=m\left[1 / 4\left(R^{2}+r^{2}\right)+h^{2 / 3}\right] \\
I_{z}=m\left(R^{2}+r^{2}\right) / 2
\end{gathered}
$$

\section{Hence}

$$
\dot{\mathrm{m}}=-4 \rho \mathrm{h} \pi \mathrm{r} \dot{\mathrm{r}}
$$

It is relatively easy to show that

$$
\dot{\mathrm{I}}=\dot{\mathrm{m}}\left(\mathrm{r}^{2 / 2}+\mathrm{h}^{2 / 3}\right)
$$

and

$$
\dot{\mathrm{I}}_{7}=\dot{\mathrm{m}} \mathrm{r}^{2}
$$

To look at the behavior of the spin rate, we examine

$$
\theta(\mathrm{t})=\dot{\mathrm{I}}_{\mathrm{z}}-(\dot{\mathrm{m}} / 2) \mathrm{R}^{2}=\dot{\mathrm{m}} \mathrm{R}^{2}\left[(\mathrm{r} / \mathrm{R})^{2}-1 / 2\right]
$$

Assuming that at time $\mathrm{t}=0, \mathrm{r}=0$, we have

$$
\left.\theta(t)\right|_{t=0}=-\dot{m} R^{2 / 2}>0
$$


and at the end of the burn $\left(t \rightarrow t_{\infty}, r=R\right)$,

$$
\left.\theta(t)\right|_{t \rightarrow t_{\infty}}=\dot{m} R^{2 / 2}<0
$$

From (53), we have that

$$
\mathrm{d} \theta / \mathrm{dt}=2 \dot{\mathrm{m}} \mathrm{r} \dot{\mathrm{r}}<0
$$

between ignition and burnout. Because $r$ varies from 0 to $R$ in the time interval $\left[0, t_{\infty}\right]$ and from the physics of the problem, it is clear that $\dot{\boldsymbol{r}}>0$ in the given interval.

We thus have a situation where $\theta(t)$ has the form shown in Fig. 7

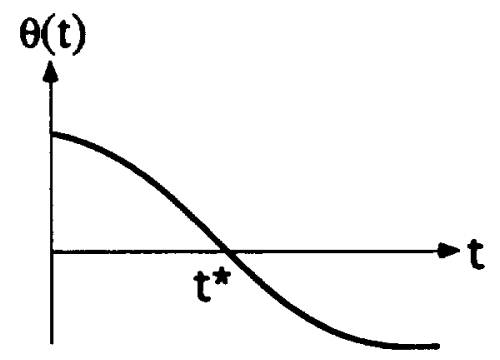

Fig. $\quad 4.7$ Shape of $\theta(t)$ for Radial Burn

The switchover point, $t^{*}$, at which the sign of $\theta(t)$ changes from positive to negative is obtained by setting $\theta(t)=0$ in $(53)$ :

$$
r / R=\sqrt{1 / 2}=0.707
$$


This implies that the spin rate decreases in the first stage of the burn, when $r / R \leq 0.707$, but increases exponentially in the later phase of the burn when $r / R>0.707$; a fact that is clearly supported by the plot shown in Fig. 8. This plot is obtained from numerical integration of (20) for centrifugal burn.

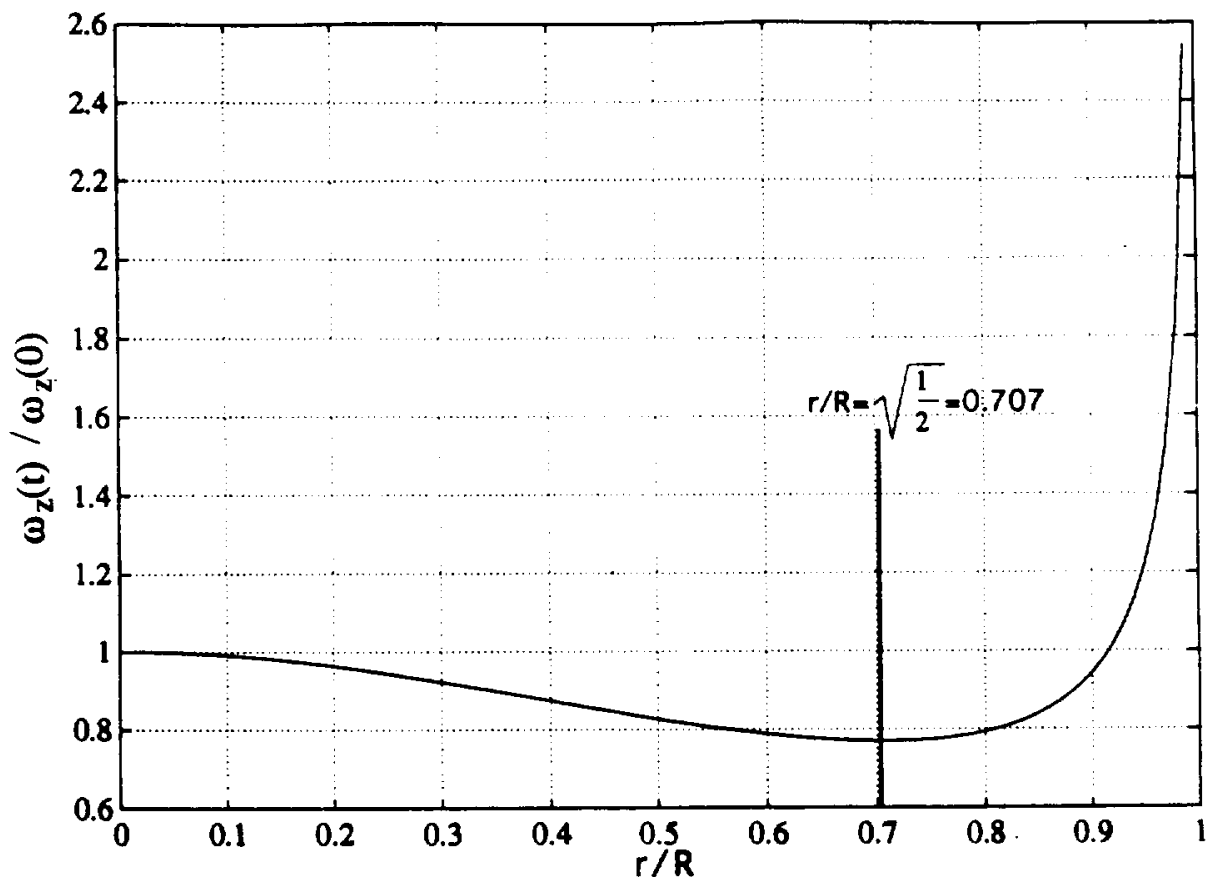

Fig. 4.8 Spin Rate Curve for Centrifugal Bum

We now discuss the evolution of the magnitude of the transverse angular velocity, $\omega_{x y}$

$$
\phi(t)=\dot{I}-\dot{m}\left(\ell^{2}+R^{2} / 4\right)=\dot{m} R^{2}\left[(1 / 2)(r / R)^{2}-(2 / 3)(h / R)^{2}-1 / 4\right]
$$

Hence

$$
\left.\phi(t)\right|_{t=0}=\phi_{0}>0
$$


and

$$
\phi_{\infty}=\dot{\mathrm{m}} \mathrm{R}^{2}\left[1 / 4-(2 / 3)(\mathrm{h} / \mathrm{R})^{2}\right] \quad
$$

From (58),

$$
\mathrm{d} \phi / \mathrm{dt}=\dot{\mathrm{m}} \mathrm{r} \dot{\mathrm{r}}<0
$$

The sign of $\phi_{\infty}$ depends on what can be described as the cylinder's "shape factor"- the ratio of the initial value of its diameter to its length. Hence, whether $\omega_{x y}$ is bounded or unbounded depends on this shape factor. To determine the value of $R / h$ that separates the "stable" region of $\omega_{x y}$ from the "unstable" region, we set $\phi(t)$ to zero in (60), and obtain

$$
\mathrm{R} / \mathrm{h}=\sqrt{8 / 3}=1.63
$$

Relevant "pictures" for $\phi(t)$ are shown in Fig. 9.

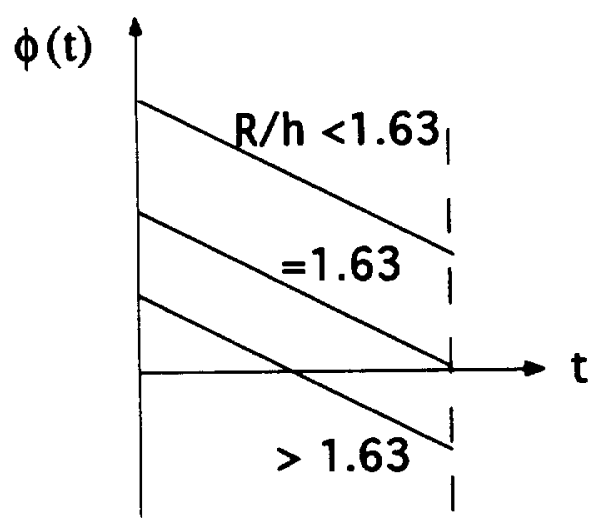

Fig. 4.9 Shape of $\phi(t)$ for Radial Burn 
Hence, the magnitude of the transverse angular velocity of a cylinder in radial burn always decreases with time in the early phases of the burn. If the ratio of the unburned cylinder's diameter to its length (shape factor) is less than that of 1.63 , the decrease of $\omega_{x y}$ continues all the way to burnout. On the other hand, if the cylinder's shape factor is greater than 1.63 , then there comes a time during the burn when $\omega_{x y}$ levels off, and then starts increasing exponentially through burnout. The initial shape of the cylinder thus has a critical effect on the cylinder's lateral attitude motion for radial burn. Pencil-shaped cylinders will tend to be stable in radial burn while hamburger-shaped cylinders will tend to be unstable. We note here that the same shape factor that determines stability or instability in radial burn was shown to influence the rate of convergence of $\omega_{x y}$ for end-burning cylinders.

It would be useful to determine the onset of instability of $\omega_{x y}$ for cases where $R / h$ $>$ 1.63. This can be done by determining the value of $r / R$ at which the sign of $\phi(t)$ changes from positive to negative in (58). We thus have, from (58), that

$$
\left.\frac{r}{R}\right|_{\phi=0}=\sqrt{\frac{4}{3}\left(\frac{h}{R}\right)+\frac{1}{2}}
$$

So, the onset of instability for $\omega_{x y}$ depends again on the shape factor. Once a value is specified for the shape factor $(R / h)$, the value of $r / R$ at the beginning of instability can be determined. For example, for

$$
\frac{\mathrm{R}}{\mathrm{h}}=\left\{\begin{array}{l}
2 \\
5,\left.\frac{\mathrm{r}}{\mathrm{R}}\right|_{\phi=0}=\left\{\begin{array}{l}
0.913 \\
0.744 \\
0.707
\end{array}\right.
\end{array}\right.
$$


We conclude, from (64), that $\omega_{x y}$ begins to diverge much earlier for cylinders with higher values of the shape factor (fat and short cylinders) than for cylinders with lower values of $R / h$. Numerically obtained plots of the transverse angular velocity are shown in Fig. 10, and are totally consistent with the above inferences. Furthermore, the above analyses capture a great deal more of the vital characteristics of radial burn than do these plots and all previous work on the cylinder.

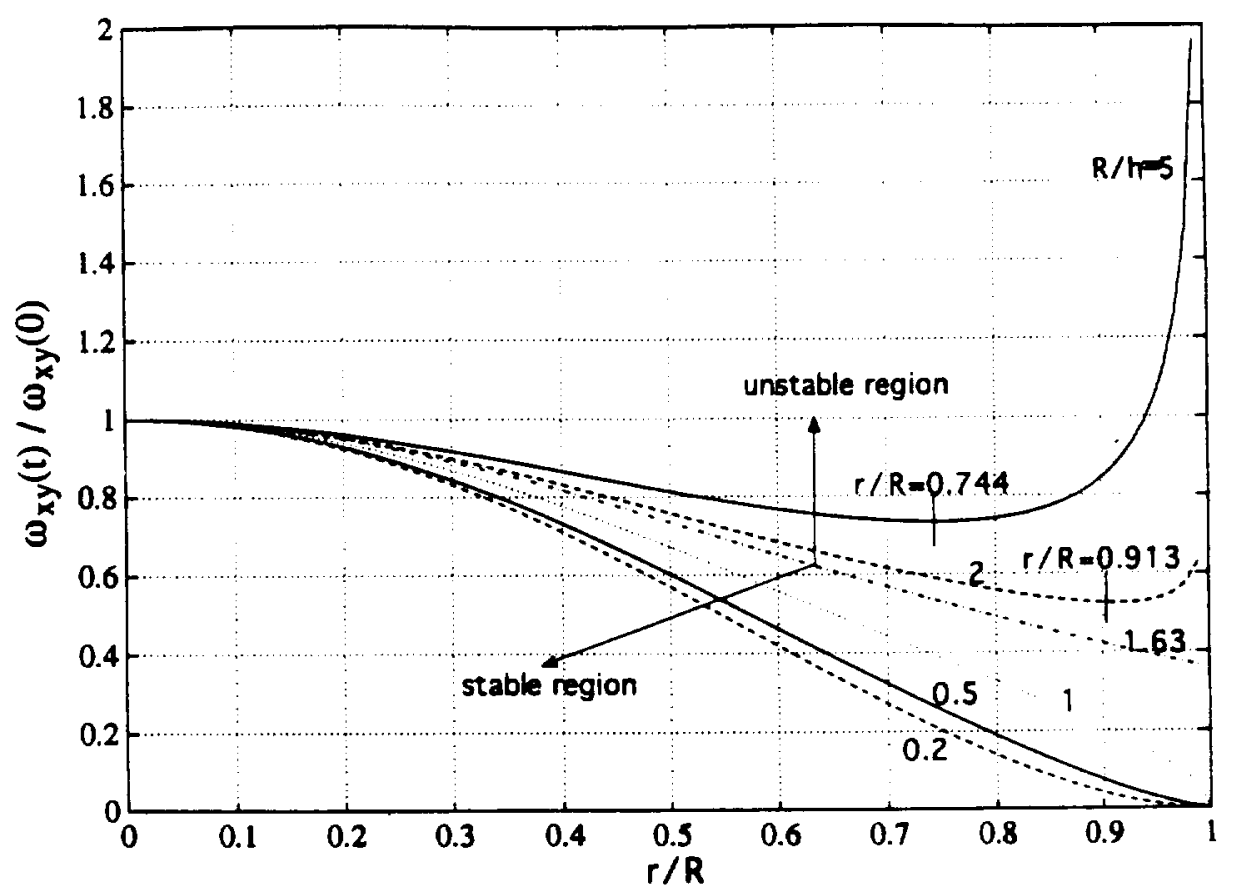

Fig. 4.10 Transverse Angular Rate for Radial Burn 


\subsubsection{Centripetal Burn}

In centripetal burn, the cylinder burns radially inwards without changing its length. The intermediate shape is a solid cylinder of diminishing radius, as shown in Fig. 11. In this case, we have that

$$
\begin{gathered}
\mathrm{m}=2 \rho \mathrm{h} \pi \mathrm{r}^{2} \\
\mathrm{I}_{\mathrm{z}}=\mathrm{m} \mathrm{r}^{2 / 2}
\end{gathered}
$$

and

$$
I=m\left(r^{2} / 4+h^{2} / 3\right)
$$

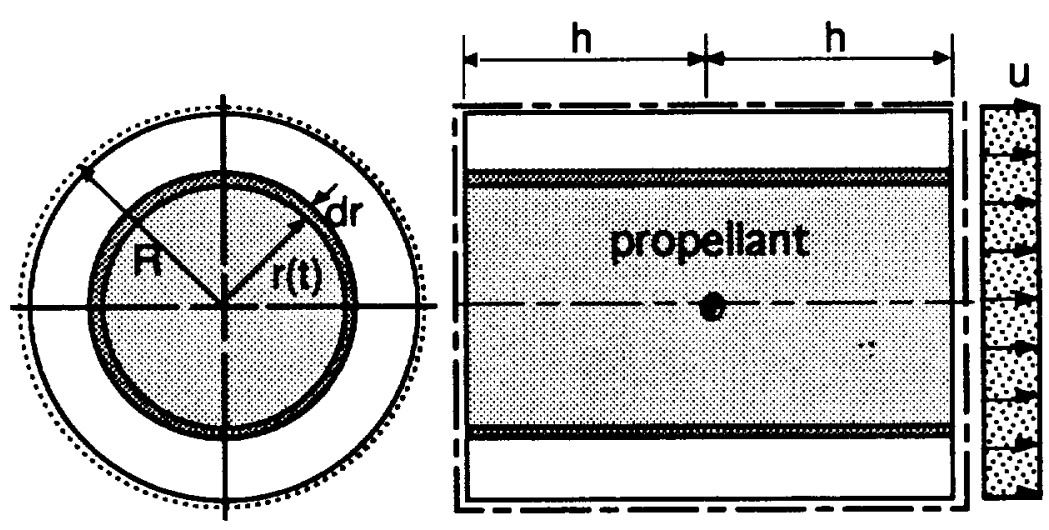

Fig. 4.11 Cylinder in Centripetal Burn 
The time derivatives are

$$
\begin{gathered}
\dot{\mathrm{m}}=4 \rho \mathrm{h} \pi \mathrm{r} \dot{\mathrm{r}} \\
\dot{\mathrm{I}}_{7}=\dot{\mathrm{m}} \mathrm{r}^{2}
\end{gathered}
$$

and

$$
\dot{\mathrm{I}}=\dot{\mathrm{m}}\left(\mathrm{r}^{2 / 2}+\mathrm{h}^{2 / 3}\right)
$$

For a study of the spin rate, we have

$$
\begin{gathered}
\theta(t)=\dot{\mathrm{I}}_{\mathrm{z}}-(1 / 2)\left(\dot{\mathrm{m}} \mathrm{R}^{2}\right)=\dot{\mathrm{m}}\left(\mathrm{r}^{2}-\mathrm{R}^{2} / 2\right) \\
\theta_{0}=\dot{\mathrm{m}} \mathrm{R}^{2} / 2<0 \\
\theta_{\infty}=-\dot{\mathrm{m}}\left(\mathrm{R}^{2} / 2\right)>0
\end{gathered}
$$

and from (71),

$$
\mathrm{d} \theta / \mathrm{dt}=2 \dot{\mathrm{m}} \mathrm{r} \dot{\mathrm{r}}>0
$$

since $\dot{\mathbf{r}}<0$ in this case. We thus have a situation where $\theta(t)$ has the form shown in Fig. 12 


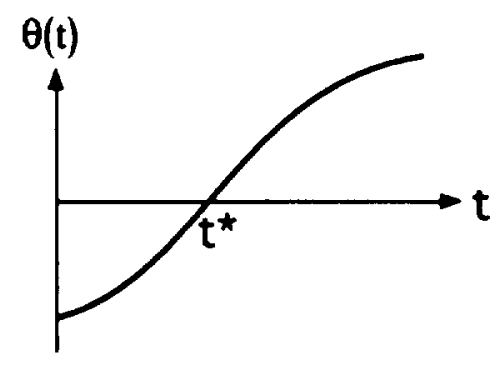

Fig. 4.12 Shape of $\theta(t)$ for Centripetal Burn

Hence the spin rate increases initially, attains a peak value then decreases rapidly with the burn. The time of attaining the peak value of the spin rate corresponds to $\theta(t)=0$. That is

$$
r / R=1 / \sqrt{2}=0.707
$$

This trend is validated by the plot of Fig. 13 below.

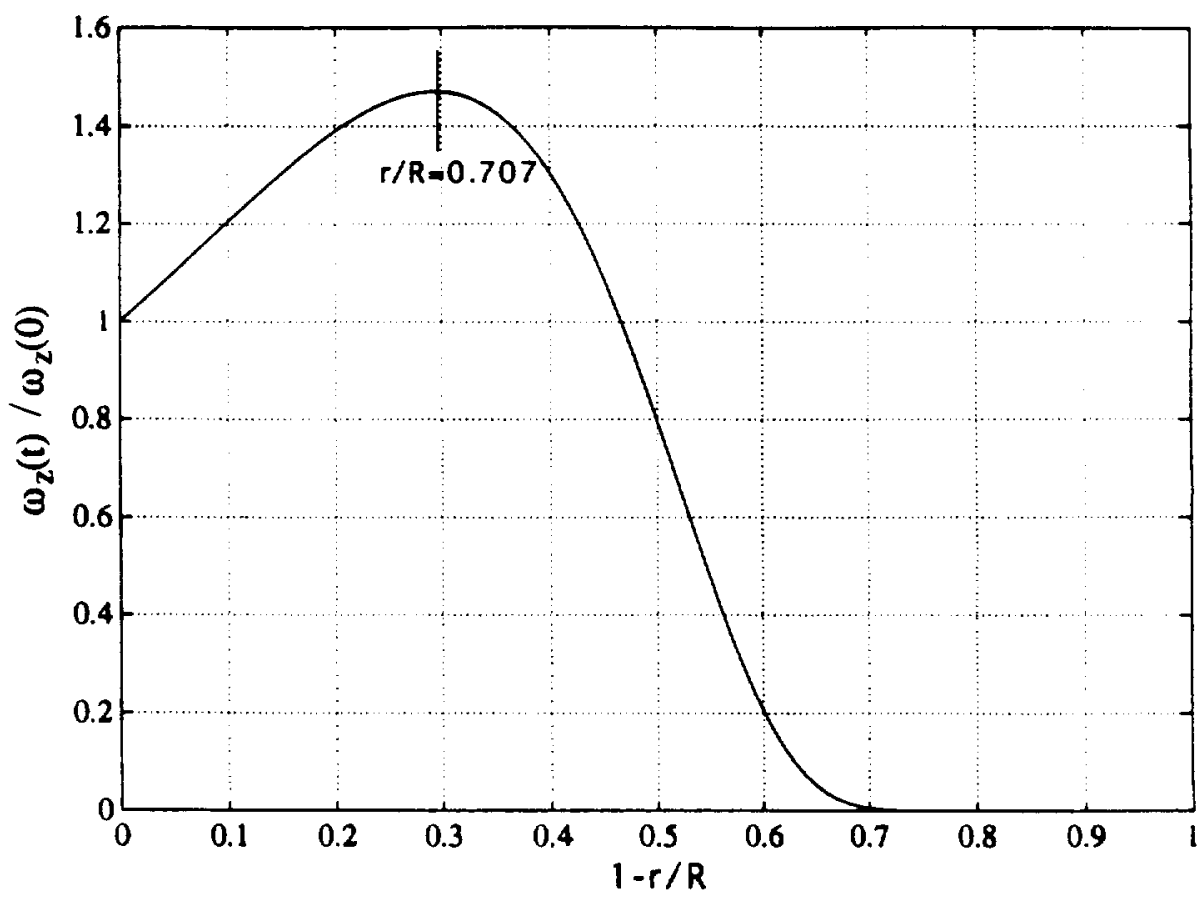

Fig. 4.13 Spin Rate of Centripetal Burn [Numerical Solution] 
To assess the evolution of the transverse angular velocity magnitude, we find

$$
\phi(\mathrm{t})=\dot{\mathrm{I}}-\dot{\mathrm{m}}\left(\ell^{2}+\mathrm{R}^{2} / 4\right)=\dot{\mathrm{m}} \mathrm{R}^{2}\left[(1 / 2)(\mathrm{r} / \mathrm{R})^{2}-(2 / 3)(\mathrm{h} / \mathrm{R})^{2}-1 / 4\right]
$$

which is exactly the same as (58) for radial bum.

$$
\begin{gathered}
\phi_{0}=\dot{\mathrm{m}} \mathrm{R}^{2}\left[1 / 4-(2 / 3)(\mathrm{h} / \mathrm{R})^{2}\right] \\
\phi_{\infty}=-\dot{\mathrm{m}} \mathrm{R}^{2}\left[1 / 4+(2 / 3)(\mathrm{h} / \mathrm{R})^{2}\right]>0
\end{gathered}
$$

and from (76)

$$
\mathrm{d} \phi / \mathrm{dt}=\dot{\mathrm{m}} \mathrm{r} \dot{\mathrm{r}}>0
$$

From (77), we determine that $\phi_{0}=0$ when $R / h=1.63, \phi_{0}>0$ for $R / h<1.63$, and $\phi_{0}<0$ for $\mathrm{R} / \mathrm{h}>1.63$. We conclude from all of this that (a) as long as the ratio of the cylinder's initial diameter to its length is less than 1.63 , the transverse angular velocity magnitude decreases as the burn progress; (b) if the ratio $R / h$ is greater than 1.63 , the magnitude of the transverse angular velocity increases initially until it reaches a peak value, then it decreases with time for the reminder of the burn. Thus $\omega_{x y}$ never really becomes unbounded for centripetal burn. These facts are demonstrated below in Fig. 14.

Our findings so far indicate that the stability of rotational motion of a variable mass cylinder depends on two factors : the burn pattern and the cylinder's shape factor. Both the spin rate and transverse angular velocity are bounded for all shape factors when the cylinder is subjected to uniform, end, or centripetal burn. For radial or centrifugal burn, the spin rate is always unbounded, while the transverse angular speed is bounded for the shape 
factor $(R / h)$ that are less then 1.63 , and unbounded for shape factors that are greater than 1.63.

All the results obtained in this section are totally consistent with, and augment considerably, earlier results by Eke and Wang(1995).

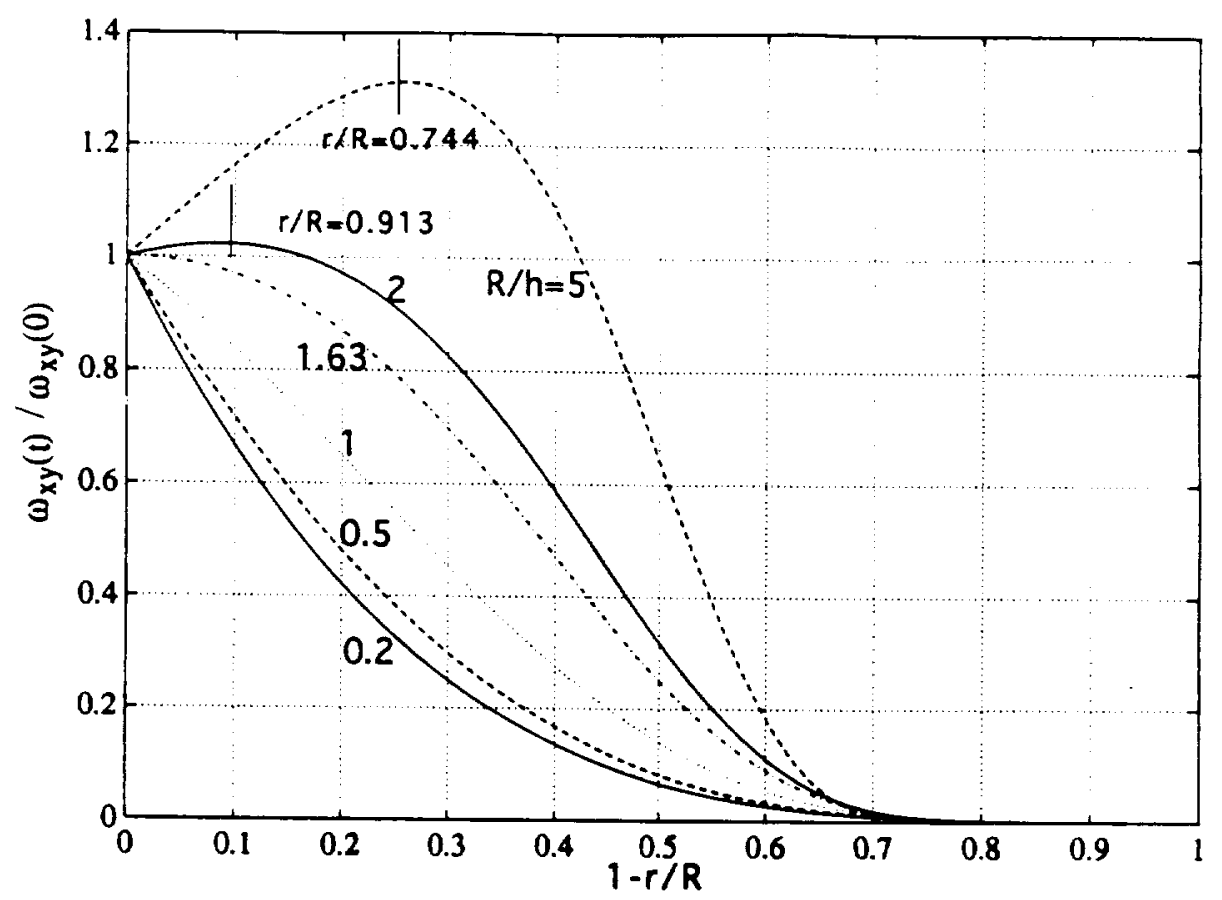

Fig. 4.14 Transverse Angular Rate of Centripetal Bum [Nümerical Results]

\subsection{Time Dependence of Motion and Inertia Properties}

Up to this point, all variables and parameters of interest in this study have been defined or determined as functions of the auxiliary variable $z$ or $r$. This approach turned out to be a very astute and efficient analytical strategy. Through this choice, we have been able to avoid some of the myriad problems encountered by previous investigators of the dynamics of variable mass systems. For example, practically all previous investigators 
worked directly with time as independent variable. Hence, to make progress with the equations of motion [ for example (11)-(13)], it was necessary to specify the time dependence of the mass $(m)$, the location of center of mass $(\ell)$ and moments of inertia ( $\left.I, I_{2}\right)$. These investigators simply made assumptions such as linear mass variation $\mathrm{m}=\mathrm{m}_{0}-\alpha \mathrm{t}$, and made "reasonable" guesses for the coefficients. They made similar guesses for $\ell, I$ and $I_{z}$. The problem is that these inertia quantities are interrelated; a fact that is generally not correctly reflected in the guesses for the independent time functions assigned to these quantities. All such difficulty is avoided by the clean analytical scheme of judiciously introducing the auxiliary variables $\mathrm{z}$ or $\mathrm{r}$, as done in this study.

Although avoiding the explicit use of time in our analysis so far has been quite beneficial from analytical point of view, it is in fact still desirable to know how the angular rates and even the inertia properties vary with time. For example, this enables us to compare analytically predicted trends with experimental results, obtained through on-board instrumentation. In this section, we show how some of the results obtained so far can be converted to time functions.

\subsubsection{End Burning Case}

We now reexamine some of the results obtained for the end burning cylinder. From (33) and (42), we have that

$$
\dot{\mathbf{z}}=-\mathbf{u} / 2
$$

or 


$$
\int_{b}^{z} d z=-\frac{u}{2} \int_{0}^{t} d t
$$

which leads to

$$
z=-(u / 2) t+h
$$

The inertia properties can now be converted to time functions simply by substituting (82) into their expressions. Thus, from (32) and (82)

$$
m=2 \rho \pi R^{2} z=2 \rho \pi R^{2}\left(h-\frac{1}{2} u t\right)=m_{0}+\dot{m} t
$$

where

$$
m_{0}=2 \rho \pi R^{2} h
$$

and $\dot{m}$ is as given in (42). Similarly,

$$
\begin{gathered}
I=m\left(R^{2 / 4}+z^{2 / 3}\right)=\left(m_{0}+\dot{m} t\right)\left[R^{2 / 4}+(h-u t / 2)^{2} / 3\right] \\
=I_{0}+P t-Q t^{2}+S t^{3}
\end{gathered}
$$

where

$$
\begin{gathered}
\mathrm{I}_{\mathrm{O}}=\mathrm{m}_{\mathrm{O}}\left(\frac{\mathrm{R}^{2}}{4}+\frac{\mathrm{h}^{2}}{3}\right) \\
\mathrm{P}=\dot{\mathrm{m}}\left(\frac{\mathrm{R}^{2}}{4}+\mathrm{h}^{2}\right) \\
Q=\frac{1}{2} \dot{\mathrm{m}} \mathrm{h} u
\end{gathered}
$$


and

$$
\mathrm{S}=\frac{1}{12} \dot{\mathrm{m}} \mathrm{u}^{2}
$$

The value of $t_{\infty}, i$. e. the burnout time, is obtained by setting $z=0$ in (82) :

$$
t_{\infty}=2 \mathrm{~h} / \mathrm{u}
$$

which is independent of the radius of the cylinder. The instantaneous transverse central radius of gyration, $\mathbf{k}$, is given by

$$
I=m k^{2}
$$

So, from (85)

$$
\mathrm{k}^{2}=\frac{\mathrm{R}^{2}}{4}+\frac{\mathrm{h}^{2}}{3}-\frac{\mathrm{hu}}{3} \mathrm{t}+\frac{\mathrm{u}^{2}}{12} \mathrm{t}^{2}
$$

Since

$$
\begin{gathered}
\mathrm{k}_{0}^{2}=\frac{\mathrm{R}^{2}}{4}+\frac{\mathrm{h}^{2}}{3} \\
\mathrm{k}^{2}=\mathrm{k}_{0}^{2}-\frac{\mathrm{hu}}{3} \mathrm{t}+\frac{\mathrm{u}^{2}}{12} \mathrm{t}^{2}
\end{gathered}
$$

For the time interval $0 \leq \mathrm{t} \leq 2 \mathrm{~h} / \mathrm{u}, \mathrm{k}^{2}$ is a decreasing quadratic function of time. At burnout 


$$
\mathrm{k}_{\infty}^{2}=\mathrm{R}^{2 / 4}
$$

The axial moment of inertia is

$$
I_{z}=\frac{1}{2} m R^{2}=\frac{1}{2} R^{2}\left(m_{o}+\dot{m} t\right)
$$

and the axial radius of gyration is a constant. Since $\dot{m}$ and $u$ are constants, we see that the transverse moment of inertia is a cubic function of time while both the axial inertia and the mass are linear functions of time for the end burn assumption.

\subsubsection{Centrifugal Burn}

For centrifugal burn, we have, from (47), that

$$
\dot{\mathrm{m}}=-2 \rho \mathrm{h} \pi \frac{\mathrm{d}\left(\mathrm{r}^{2}\right)}{\mathrm{dt}}=-\rho \pi \mathrm{R}^{2} \mathrm{u}
$$

Hence

$$
\frac{\mathrm{d}\left(\mathrm{r}^{2}\right)}{\mathrm{dt}}=\frac{\mathrm{R}^{2} \mathrm{u}}{2 \mathrm{~h}}
$$

Integrating both sides over appropriate limits, we have

$$
\mathrm{r}^{2}=\frac{\mathrm{R}^{2} \mathrm{u}}{\mathrm{T} \cdot \mathrm{h}} \mathrm{t}
$$


Hence,

$$
\begin{aligned}
m=2 & \rho h \pi\left(R^{2}-r^{2}\right)=2 \rho h \pi\left[R^{2}-\left(R^{2} u t\right)(2 h)\right]=m_{0}+\dot{m} t \\
I & =m\left(\frac{R^{2}+r^{2}}{4}+\frac{h^{2}}{3}\right)=\left(m_{0}+\dot{m} t\right)\left(\frac{R^{2}}{4}+\frac{R^{2} u}{8 h} t+\frac{h^{2}}{3}\right) \\
& =I_{0}+B t+A t^{2}
\end{aligned}
$$

where

$$
\begin{gathered}
I_{0}=m_{0}\left(R^{2 / 4}+h^{2 / 3}\right) \\
A=\left(\dot{m} R^{2} u\right) /(8 h) \\
B=\dot{m} h^{2 / 3}
\end{gathered}
$$

Since both $A$ and $B$ are negative, it is clear from (101) that $I$ is a decreasing quadratic function of time. Note also that

$$
\dot{I}=B+2 A t<0
$$

From (49) 


$$
\begin{aligned}
I_{z} & =\frac{1}{2} m\left(R^{2}+r^{2}\right)=\frac{1}{2}\left(m_{0}+\dot{m} t\right)\left(R^{2}+\frac{R^{2} u t}{2 h}\right) \\
& =I_{z o}+2 A t^{2}
\end{aligned}
$$

and

$$
\dot{\mathrm{I}}_{7}=4 \mathrm{At}<0
$$

where

$$
I_{z 0}=\frac{1}{2} m_{0} R^{2}
$$

Thus, $I_{z}$ is also a decreasing quadratic function of time.

It is straightforward to show that the radii of gyration are given by

$$
k^{2}=k_{0}^{2}+\frac{R^{2} u}{8 h} t
$$

and

$$
k_{z}^{2}=k_{z 0}^{2}+\frac{R^{2} u}{4 h} t
$$

where

$$
k_{0}^{2}=\frac{R^{2}}{4}+\frac{h^{2}}{3}
$$

and

$$
\mathrm{k}_{\mathrm{zO}}^{2}=\frac{\mathrm{R}^{2}}{2}
$$

So, $\mathrm{k}^{2}$ and $\mathrm{k}_{\mathrm{z}}^{2}$ are both linearly increasing functions of time. 
Now, the value of burnout time $t_{0}$ is obtained by setting $r=R$ in (99). Hence

$$
t_{\infty}=\frac{2 \cdot h}{u}
$$

and

$$
\begin{gathered}
k_{\infty}^{2}=\left.k^{2}\right|_{t=\frac{2 h}{u}}=\frac{R^{2}}{2}+\frac{h^{2}}{3} \\
k_{z \infty}^{2}=R^{2}
\end{gathered}
$$

\subsubsection{Centripetal Burn}

In the case of centripetal burn, similar analyses lead to

$$
\begin{gathered}
r^{2}=R^{2}-\frac{R^{2} u}{7 \cdot h} t \\
t_{\infty}=\frac{2 \cdot h}{u} \\
m=m_{0}+\dot{m} \cdot t \\
I=I_{0}+C t-A t^{2}
\end{gathered}
$$

with

$$
\mathrm{C}=\dot{\mathrm{m}}\left(\frac{\mathrm{R}^{2}}{2}+\frac{\mathrm{h}^{2}}{3}\right)
$$




$$
\begin{gathered}
\dot{I}=C-2 A t \\
I_{z}=I_{z 0}+D t-2 A t^{2}
\end{gathered}
$$

where

$$
\mathrm{D}=\dot{\mathbf{m}} \mathbf{R}^{2}
$$

Hence, both $I$ and $I_{z}$ are decreasing quadratic functions of time.

The radii of gyration can also be shown to be given by

$$
k^{2}=k_{0}^{2}-\frac{R^{2} u}{8 h} \cdot t
$$

and

$$
k_{z}^{2}=k_{\mathrm{zO}}^{2}-\frac{R^{2} u}{4 h} \cdot t
$$

Both $\mathbf{k}^{2}$ and $\mathbf{k}_{\mathbf{z}}^{2}$ are decreasing linear functions of time. We also have that

$$
k_{\infty}^{2}=h^{2 / 3}
$$

and

$$
\mathbf{k}_{\mathrm{Z} \infty}^{2}=0
$$

In conclusion, we observe that for the three cases examined, the cylinder mass is a linear function of time and the burn duration is the same for all cases. For the end burning cylinder, $I$ is a cubic function of time, while $k^{2}$ is a quadratic function of time; for centrifugal and centripetal burns, $I$ is a quadratic while $\mathbf{k}^{2}$ is linear in time. All of the inertia 
parameters are decreasing functions of time except for centrifugal burn where the radius of gyration increases with time. 


\section{CHAPTER 5}

\section{MOTION OF AXISYMMETRIC BODIES WITH MASS LOSS}

In the previous chapter, the important problem of the attitude stability of rocket systems is initiated, with such systems idealized by a right circular cylinder. In particular, the effects of mass loss for various burning patterns are emphasized.

In this chapter, another step in the study of the attitude motions of rockets is taken by considering a more sophisticated model as shown in Fig. 1 below. The system investigated here differs from the cylinder model in two basic ways : (i) the system is modeled as a general axisymmetric system (not just a cylinder), and includes a cylindrical solid propellant grain as well as a constant mass, non-propellant portion; (ii) a nozzle arrangement is also included. The new system can be reduced to the cylinder model by setting the mass of the invariant part to zero.

The objectives in this chapter are similar to those of the previous chapter, we are interested in the angular rates of the system, and how they are influenced by mass loss. 


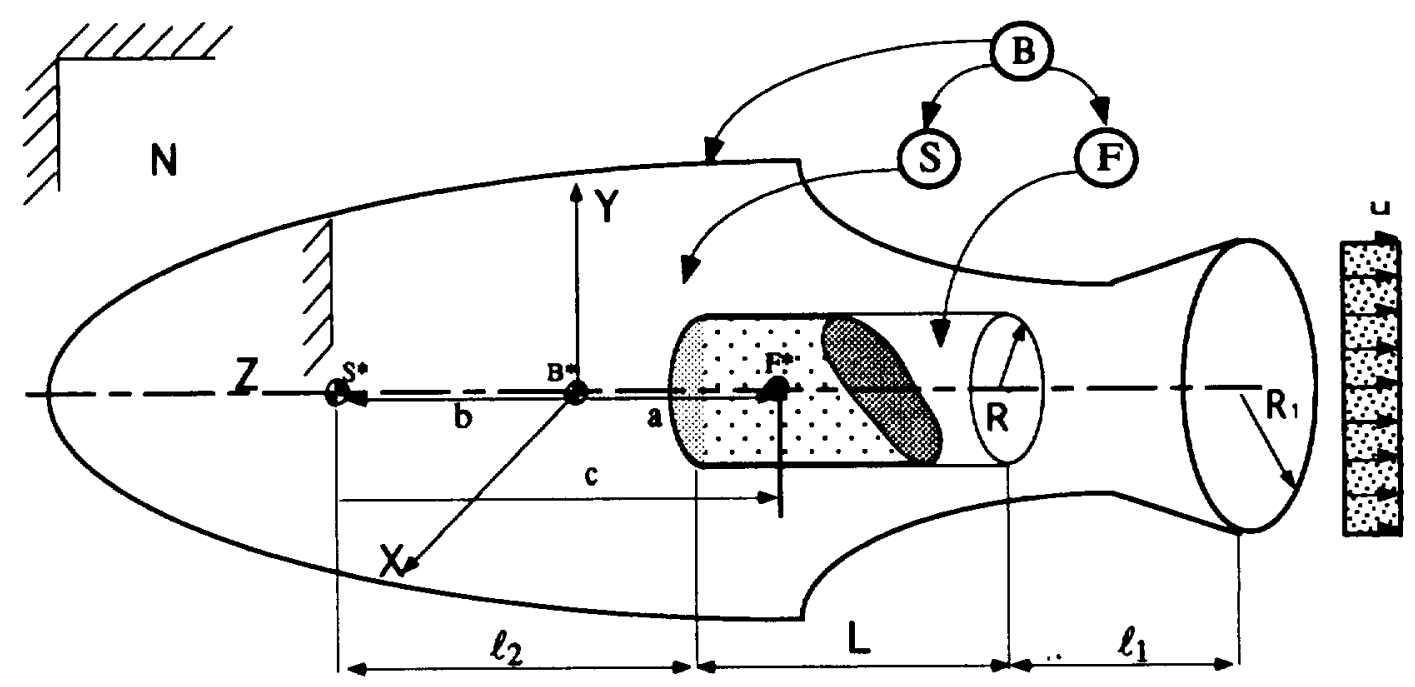

Fig. 5.1 Axisymmetric Solid Rocket System

\subsection{Governing Equations}

The system under consideration in this chapter is shown in Fig. 1, and consists of two main parts, $S$ and $F$. F represents the solid propellant of a typical rocket system. It is assumed to be rigid and cylindrical. $S$ is the main part of the system excluding fuel and includes the payload $S$ is assumed to be rigid and axisymmetric, and maintains the same mass throughout. Both $\mathrm{S}$ and $\mathrm{F}$ have the same axis of symmetry, so that the mass canters $S^{*}$ of $S, F^{*}$ of $F$ and $B^{*}$ of the combined system, all lie on the common axis of symmetry z. The system loses mass continuously through a planar portion of its surface that we shall refer to as the nozzle exit plane, and that is located at the right end of the system in Fig. 1. At any given instant, the overall system will consist of everything that is bounded by the external boundary of $S$ and the nozzle exit plane.

System dimensions are characterized by the symbols given in Fig. 1. Ms represents the mass of the invariant part, $S$, and $m_{f}$ is the instantaneous mass of the fuel, $F$. The origin of the coordinate axes $x, y, z$ is located at, and moves with, the composite mass 
center $B^{*}$, such that the $z$-axis is always aligned with the symmetry axis, and the $x$ and $y$ axes have no rotation relative to $S$. The solid propellant, $F$, is assumed to maintain an axisymmetric configuration throughout its burn, so that the central inertia matrices for $S$ and $\mathrm{F}$ are diagonal. Externally applied moments, thrust misalignment effects, aerodynamic and gravitational moments are assumed to be of relatively minor importance during burning, and are thus neglected. The system is thus assumed to move in a torque-free environment. The motion of the particles of the products of combustion as they cross the exit plane is taken to be such that the velocity distribution perpendicular to the plane is uniform. If it is further assumed that the motion of gas particles inside the combustion chamber is symmetric relative to the z-axis, and that whirling motion of the gas particles within the system is negligible, the vector equation of attitude motion for the system is reduced from (2.52) to

$$
\tilde{\mathbf{I}} \cdot \boldsymbol{\alpha}+\boldsymbol{\omega} \times \tilde{\mathbf{I}} \cdot \omega+\left(\frac{{ }^{B}}{d t} \tilde{\mathbf{I}}\right) \cdot \omega+\int_{S} \rho[\mathbf{p} \times(\omega \times \mathbf{p})]\left(\mathbf{v}_{r} \cdot \mathbf{n}\right) d S=0
$$

Because of the symmetry assumption, the central inertia dyadics for $F, S$, and the overall system B can be written respectively as

$$
\begin{aligned}
& \tilde{\mathbf{I}}_{\mathrm{f}}=\mathrm{I}_{\mathrm{f}}\left(\mathbf{b}_{\mathbf{x}} \mathbf{b}_{\mathbf{x}}+\mathbf{b}_{\mathbf{y}} \mathbf{b}_{\mathbf{y}}\right)+\mathrm{J}_{\mathrm{f}} \mathbf{b}_{\mathbf{z}} \mathbf{b}_{\mathbf{z}} \\
& \tilde{\mathbf{I}}_{s}=I_{s}\left(b_{x} b_{x}+b_{y} b_{y}\right)+J_{s} b_{z} b_{z}
\end{aligned}
$$

and 


$$
\tilde{\mathbf{I}}=\mathbf{I}\left(\mathbf{b}_{\mathbf{x}} \mathbf{b}_{\mathbf{x}}+\mathbf{b}_{\mathbf{y}} \mathbf{b}_{\mathbf{y}}\right)+\mathbf{J b}_{\mathbf{z}} \mathbf{b}_{\mathbf{z}}
$$

We also have that

$$
I=I_{s}+I_{f}+M_{s} b^{2}+m_{f} a^{2}
$$

and

$$
\mathrm{J}=\mathrm{J}_{\mathrm{s}}+\mathrm{J}_{\mathrm{f}}
$$

Note also that the distances $a$ and $b$ of Fig. 1 are given respectively by

$$
\begin{aligned}
& a=\frac{M_{s}}{\left(M_{s}+m_{f}\right)} c \\
& b=\frac{m_{f}}{\left(M_{8}+m_{f}\right)} c
\end{aligned}
$$

As propellant mass is lost, the system mass center $B^{*}$ moves forward toward $S^{*}$. Combining (5), (7) and (8), we have

$$
I=I_{s}+I_{f}+\mu c^{2}
$$

where 


$$
\mu=\frac{m_{f} M_{s}}{\left(M_{s}+m_{f}\right)}
$$

Letting the inertial angular velocity of $\mathrm{S}$ be

$$
\omega=\omega_{x} b_{x}+\omega_{y} b_{y}+\omega_{z} b_{z}
$$

the inertial angular acceleration is

$$
\alpha=\dot{\omega}_{x} b_{x}+\dot{\omega}_{y} b_{y}+\dot{\omega}_{z} b_{z}
$$

(4) and (12) then give

$$
\tilde{\mathbf{I}} \cdot \alpha=\mathrm{I}\left(\dot{\omega}_{\mathrm{x}} \mathbf{b}_{\mathrm{x}}+\dot{\omega}_{\mathrm{y}} \mathbf{b}_{\mathrm{y}}\right)+\mathrm{J} \dot{\omega}_{\mathrm{z}} \mathbf{b}_{\mathrm{z}}
$$

and (4), and (11) lead to

$$
\omega \times \tilde{\mathbf{I}} \cdot \omega=(\mathrm{J}-\mathrm{I}) \omega_{\mathrm{z}}\left(\omega_{\mathrm{y}} \mathbf{b}_{\mathrm{x}}-\omega_{\mathrm{x}} \mathbf{b}_{\mathrm{y}}\right)
$$


We also have from (4) and (11) that

$$
\left(\frac{s}{d \tilde{I}}\right)-\omega=\dot{I}\left(\omega_{x} b_{x}+\omega_{y} b_{y}\right)+\dot{J} \omega_{z} b_{z}
$$

Finally, because of the simplifying assumption made so far,

$$
\int_{S_{B}} \rho \mathbf{r} \times(\omega \times \mathbf{r})\left(\mathbf{v}_{\mathrm{r}} \cdot \mathbf{n}\right) \mathrm{d} S=-\dot{\mathrm{m}}_{\mathrm{f}}\left[\left(\ell_{\mathrm{e}}^{2}+\mathrm{R}_{1}^{2} / 4\right)\left(\omega_{\mathrm{x}} \mathbf{b}_{\mathrm{x}}+\omega_{\mathrm{y}} \mathbf{b}_{\mathrm{y}}\right)+\left(\mathrm{R}_{1}^{2} / 2\right) \omega_{\mathrm{z}} \mathbf{b}_{\mathrm{z}}\right]
$$

where $\ell_{e}$ is the axial distance between $F^{*}$ and the nozzle exit plane. By combining (1) and (13)-(16), we have that the scalar equations of attitude motion for the system are

$$
\begin{gathered}
I \dot{\omega}_{\mathrm{x}}+(\mathrm{J}-\mathrm{I}) \omega_{\mathrm{y}} \omega_{\mathrm{z}}+\left[\dot{\mathrm{I}}-\dot{\mathrm{m}}_{\mathrm{f}}\left(\ell_{\mathrm{e}}^{2}+\mathrm{R}_{1}^{2} / 4\right)\right] \omega_{\mathrm{x}}=0 \\
I \dot{\omega}_{\mathrm{y}}-(\mathrm{J}-\mathrm{I}) \omega_{\mathrm{x}} \omega_{\mathrm{z}}+\left[\dot{\mathrm{I}}-\dot{\mathrm{m}}_{\mathrm{f}}\left(\ell_{\mathrm{e}}^{2}+\mathrm{R}_{1}^{2} / 4\right)\right] \omega_{\mathrm{y}}=0 \\
\mathrm{~J} \dot{\omega}_{\mathrm{z}}+\left(\dot{\mathrm{J}}_{\mathrm{f}}-\dot{\mathrm{m}}_{\mathrm{f}} \mathrm{R}_{1}^{2} / 2\right) \omega_{\mathrm{z}}=0
\end{gathered}
$$

where I and J are given by (9) and (6) respectively, and [see (9)] 


$$
\dot{\mathrm{I}}=\dot{\mathrm{I}}_{\mathrm{f}}+\dot{\mu} \mathrm{c}^{2}+2 \mu \mathrm{c} \dot{\mathrm{c}}
$$

$$
\dot{J}=\dot{J}_{\mathbf{f}}
$$

\subsection{Non-Dimensional Equations of Motion}

In this section, we render the equations of attitude motion very compact through the introduction of a set of dimensionless parameters. This allows us to perform further studies of these equations in a way that provides great insight into the most important factors that govern the behavior of the system. We start by introducing the nondimensional time. For the system of Fig. 1, the instantaneous mass of the cylindrical propellant is

$$
m_{f}=m_{f 0}-\tilde{m} t
$$

where

$$
\widetilde{\mathrm{m}}=-\dot{\mathrm{m}}
$$


and is taken to be a positive constant that represents the rate of fuel consumption. We assume that $t=0$ at ignition, when $\mathrm{m}_{\mathrm{f}}=\mathrm{m}_{\mathrm{fO}}$, and that at burnout, $t=t_{\mathrm{b}}$ and $\mathrm{m}_{\mathrm{f}}=0$. Hence, (22) gives, at burnout,

$$
t_{b}=m_{f o} / \widetilde{m}
$$

We define a dimensionless time, $\tau$, as

$$
\tau=\frac{t}{t_{b}}=\frac{\widetilde{m}}{m_{f 0}} t=\alpha t
$$

Thus, $\tau=0$ at ignition, and $\tau=1$ at burnout. Also, the quantity $\alpha^{-1}$ has the dimension of time and is an appropriate scaling factor for time.

We have from (25) that

$$
\frac{d}{d t}=\alpha \frac{d}{d \tau}
$$

and the equations of motion, (17)-(19), can be re-written as

$$
\omega_{\mathrm{x}}^{\prime}=\frac{-(\mathrm{J}-\mathrm{I}) \omega_{\mathrm{y}} \omega_{\mathrm{z}}-\alpha\left[\mathrm{I}^{\prime}-\mathrm{m}^{\prime}\left(\ell_{\mathrm{e}}^{2}+\mathrm{R}_{1}^{2} / 4\right)\right] \omega_{\mathrm{x}}}{\mathrm{I} \alpha}
$$




$$
\begin{gathered}
\omega_{y}^{\prime}=\frac{(J-I) \omega_{x} \omega_{z}-\alpha\left[I^{\prime}-m^{\prime}\left(\ell_{e}^{2}+R_{1}^{2} / 4\right)\right] \omega_{y}}{I \alpha} \\
\omega_{z}^{\prime}=\frac{-\left[J^{\prime}-m^{\prime} R_{1}^{2} / 2\right] \omega_{z}}{J}
\end{gathered}
$$

where the prime indicates derivative with respect to $\tau$, and the inertia properties $I, J$ and $\mathrm{m}_{\mathrm{f}}$ are assumed to be expressed in terms of $\tau$.

In the next phase of the non-dimensionalization process, we use $\mathrm{m}_{\mathrm{fo}}$ and $\mathrm{R}$ as scale factors for mass and length respectively, so that the scaling factor for inertia scalars becomes $m_{f n} R^{2}$. It is also convenient to introduce, at this point, the following nondimensional parameters :

$$
\left.\begin{array}{c}
\gamma_{1}=\mathbf{k}_{\mathrm{s}} / \mathrm{R}, \gamma_{2}=\mathrm{k}_{\mathrm{sz}} / \mathrm{R}, \psi=\mathrm{M}_{\mathrm{s}} / \mathrm{m}_{\mathrm{o}}, \delta=\mathrm{L} / \mathrm{R} \\
\beta=\mathrm{R}_{1} / \mathrm{R}, \delta_{1}=\ell_{1} / \mathrm{R}, \delta_{2}=\ell_{2} / \mathrm{R}
\end{array}\right\}
$$

where $k_{\mathbf{s}}$ and $k_{\mathbf{s z}}$ are, respectively the transverse and axial radius of gyration of $S$.

We now divide the numerator and denominator of the right hand side of (27) by $m_{m} R^{2}$ to obtain 


$$
\omega_{\mathrm{x}}=\frac{-\left(\frac{\mathrm{J}}{\mathrm{m}_{\mathrm{fO}} \mathrm{R}^{2}}-\frac{\mathrm{I}}{\mathrm{m}_{\mathrm{f} 0} \mathrm{R}^{2}}\right) \omega_{\mathrm{y}} \omega_{\mathrm{z}}-\alpha\left[\frac{\mathrm{I}}{\mathrm{m}_{\mathrm{f} 0} \mathrm{R}^{2}}-\frac{\mathrm{m}^{\prime}}{\mathrm{m}_{\mathrm{fO}} \mathrm{R}^{2}}\left(\ell_{\mathrm{e}}^{2}+\mathrm{R}_{1}^{2} / 4\right)\right] \omega_{\mathrm{x}}}{\frac{\mathrm{I}}{\mathrm{m}_{\mathrm{fo}} \mathrm{R}^{2}} \alpha}
$$

If both sides of (31) are divided by $\alpha$, we get

$$
\bar{\omega}_{\mathrm{x}}=-\left(\frac{\bar{J}}{\bar{I}}-1\right) \bar{\omega}_{\mathrm{y}} \bar{\omega}_{\mathrm{z}}-\frac{\left\{\bar{I}^{\prime}-\frac{m^{\prime}}{m_{\mathrm{f} 0}}\left[\left(\frac{\ell_{\mathrm{e}}}{\mathrm{R}}\right)^{2}+\frac{\beta^{2}}{4}\right]\right\}}{\overline{\mathrm{I}}} \bar{\omega}_{\mathrm{x}}
$$

Similarly,

$$
\bar{\omega}_{y}=(\bar{J} / \bar{I}-1) \bar{\omega}_{x} \bar{\omega}_{z}-\frac{\left\{\bar{I}^{\prime}-\frac{m^{\prime}}{m_{f 0}}\left[\left(\frac{\ell_{\mathrm{e}}}{R}\right)^{2}+\frac{\beta^{2}}{4}\right]\right]}{\bar{I}} \bar{\omega}_{y}
$$

and

$$
\bar{\omega}_{\mathrm{z}}^{\prime}=\frac{\left(\overline{\mathrm{J}}^{\prime}-\frac{\mathrm{m}^{\prime}}{\mathrm{m}_{\mathrm{f} 0}} \frac{\beta^{2}}{2}\right)}{\overline{\mathrm{J}}} \bar{\omega}_{\mathrm{z}}
$$


where $\bar{\omega}_{x}, \bar{\omega}_{y}, \bar{\omega}_{z}$ are non-dimensionalized angular velocity components, and $\overline{\mathbf{I}}, \overline{\mathbf{J}}$ are normalized inertia scalars.

Following a strategy similar to that utilized in chapter 3, we let

$$
\phi(\tau)=\bar{I}^{\prime}-\frac{m^{\prime}}{m_{f 0}}\left[\left(\frac{\ell_{e}}{R}\right)^{2}+\frac{\beta^{2}}{4}\right]
$$

and

$$
\theta(\tau)=\overline{\mathbf{J}}^{\prime}-\frac{\mathrm{m}^{\prime}}{\mathrm{m}_{\mathrm{f}}} \frac{\beta^{2}}{2}
$$

Next, we multiply (32) by $\bar{\omega}_{\mathrm{x}}$ and (33) by $\bar{\omega}_{\mathrm{y}}$ and add :

$$
\frac{d}{d \tau}\left(\vec{\omega}_{x}^{2}+\vec{\omega}_{y}^{2}\right)=-2 \frac{\phi(\tau)}{\bar{I}}\left(\vec{\omega}_{x}^{2}+\vec{\omega}_{y}^{2}\right)
$$

(34) becomes

$$
\frac{d}{d \tau} \bar{\omega}_{z}=-\frac{\theta(\tau)}{\bar{J}} \bar{\omega}_{z}
$$


(37) and (38) lead to

$$
\bar{\omega}_{\mathrm{t}}=\bar{\omega}_{t 0} \exp \left(-\int_{0}^{1} \frac{\phi(\tau)}{\overline{\mathrm{I}}} \mathrm{d} \tau\right)
$$

and

$$
\bar{\omega}_{z}=\bar{\omega}_{z 0} \exp \left(-\int_{0}^{1} \frac{\theta(\tau)}{\bar{J}} d \tau\right)
$$

where

$$
\bar{\omega}_{t}=\sqrt{\bar{\omega}_{x}^{2}+\bar{\omega}_{y}^{2}}
$$

As in the case of the cylinder, the sign of the functions $\phi$ and $\boldsymbol{\theta}$ determine whether the angular rates grow or decay with time. A positive sign implies decay and a negative sign signals divergence.

From Fig. 1

$$
\mathrm{m}_{\mathrm{fo}}=\rho_{\mathrm{fo}} \pi \mathrm{R}^{2} \mathrm{~L}
$$


where $\rho_{\mathrm{fo}}$ is the mass density of F. Continuity equation gives

$$
\dot{\mathrm{m}}=\dot{\mathrm{m}}_{\mathrm{f}}=-\tilde{\mathrm{m}}=2 \pi \int_{0}^{R_{1}} \rho \mathrm{u}(\mathrm{r}) \mathrm{rdr}
$$

We recall here that $\rho$ is the density of the fluid products of combustion at the exit plane.

For a uniform velocity profile at the exit plane, $u(t)$ is constant, so that

$$
\dot{\mathrm{m}}=\dot{\mathrm{m}}_{\mathrm{f}}=-\widetilde{\mathrm{m}}=-\rho \pi \mathrm{R}_{1}^{2} \mathrm{u}
$$

We then have an alternate expression for $\alpha$ as

$$
\alpha=\frac{\widetilde{m}}{m_{\mathrm{o}}}=\frac{\rho \pi R_{1}^{2} u}{\rho_{\mathrm{ff}} \pi \mathrm{R}^{2} \mathrm{~L}}=\frac{\rho}{\rho_{\mathrm{fo}}}\left(\frac{\mathrm{R}_{1}}{\mathrm{R}}\right)^{2}\left(\frac{\mathrm{u}}{\mathrm{L}}\right)=\eta \beta^{2} \frac{\mathrm{u}}{\mathrm{L}}
$$

where $\eta$ is the ratio $\rho / \rho_{\mathrm{fo}}$. Note also that

$$
\frac{\beta^{2} \mathrm{u}}{\mathrm{R}}=\left(\frac{\beta^{2} \mathrm{u}}{\mathrm{L}}\right)\left(\frac{\mathrm{L}}{\mathrm{R}}\right)=\frac{\alpha \delta}{\eta}
$$


To make further progress with our analysis, we will now focus attention on two specific burn scenarios for $\mathrm{F}$ that are closest to what occurs in real rockets.

\subsection{Centrifugal Burn}

The fuel burns radially outwands in a symmetric manner (see Fig. 2) so that F* remains fixed in S; however, B* still moves toward S*. From Fig. 2,

$$
m_{f}=\rho_{f} \pi L\left(R^{2}-r^{2}\right)
$$

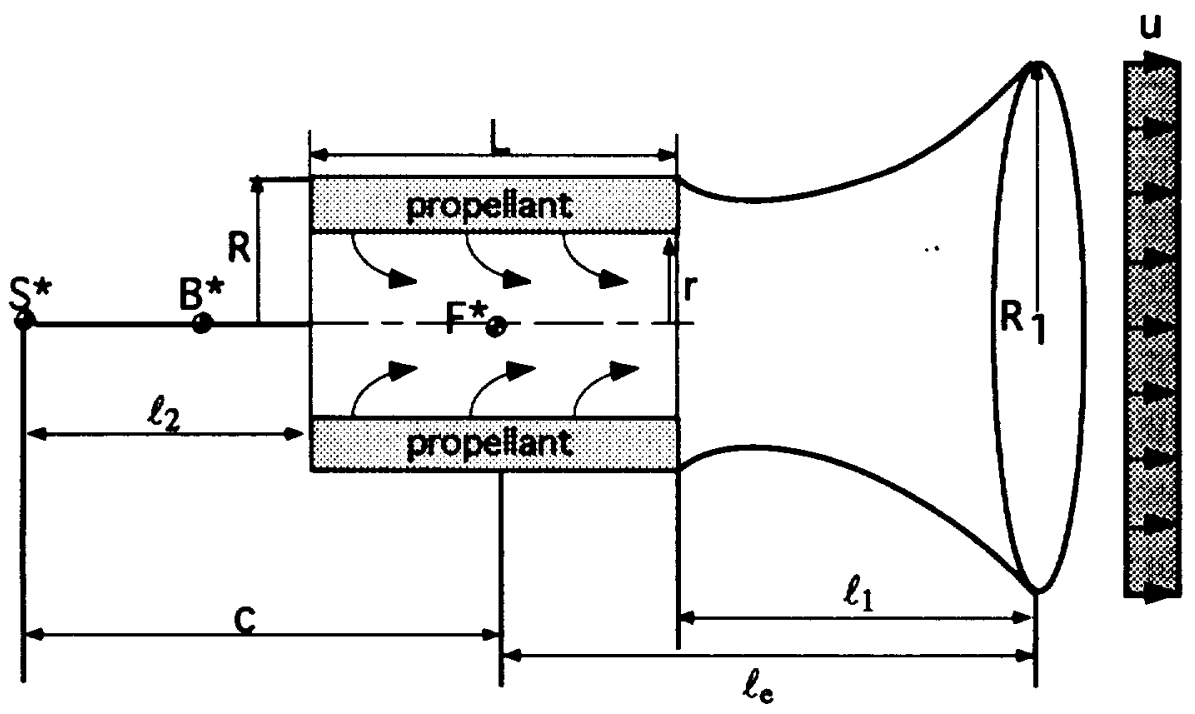

Fig. 5.2 Centrifugal Burn 
From (47) and (44)

$$
\frac{\mathrm{dm}_{\mathrm{f}}}{\mathrm{dt}}=-\rho_{\mathrm{f}} \pi \mathrm{L} \frac{\mathrm{d}\left(\mathrm{r}^{2}\right)}{\mathrm{dt}}=-\rho \pi \mathrm{R}_{1}^{2} \mathrm{u}
$$

Hence

$$
\int_{0}^{r^{2}} d\left(r^{2}\right)=\eta \frac{R_{1}^{2} u}{L} \int_{0}^{t} d t
$$

and

$$
r^{2}=\eta \frac{R_{1}^{2} u}{L} t=\eta \frac{R_{1}^{2} u}{L \alpha} \tau
$$

From (50) and (45)

$$
\frac{\mathrm{r}^{2}}{\mathrm{R}^{2}}=\eta \frac{\beta^{2} \mathrm{u}}{\mathrm{L} \alpha} \tau=\frac{\alpha}{\alpha} \tau=\tau
$$




$$
\frac{m_{f}}{m_{f 0}}=\frac{\rho_{\mathrm{f}} \pi L\left(R^{2}-r^{2}\right)}{\rho_{f} \pi L R^{2}}=1 \frac{r^{2}}{R^{2}}=1-\tau
$$

and so,

$$
\begin{gathered}
\bar{I}_{f}=\frac{I_{f}}{m_{0} R^{2}}=\frac{m_{f}}{m_{0}}\left[\frac{\left(R^{2}+r^{2}\right)}{4 R^{2}}+\frac{L^{2}}{12 R^{2}}\right]=(1-\tau)\left(\frac{1+\tau}{4}+\frac{\delta^{2}}{12}\right) \\
=\frac{1}{4}+\frac{\delta^{2}}{12}-\frac{\delta^{2} \tau}{12}-\frac{\tau^{2}}{4}
\end{gathered}
$$

from which we have

$$
\overline{\mathrm{I}}_{\mathrm{f}}^{\prime}=-\frac{\delta^{2}}{12}-\frac{\tau}{2}
$$

Similarly,

$$
\bar{J}_{f}=\frac{J_{f}}{m_{n} R^{2}}=\frac{m_{f}}{m_{0}} \frac{\left(R^{2}+r^{2}\right)}{2 R^{2}}=(1-\tau) \frac{1+\tau}{2}=\frac{\left(1-\tau^{2}\right)}{2}
$$

and

$$
\overline{\mathbf{J}}_{\mathrm{f}}^{\prime}=-\tau
$$


Because (see Fig. 2)

$$
c=\ell_{2}+L / 2
$$

we have

$$
c^{\prime}=0
$$

and

$$
2 \mu c c^{\prime}=0
$$

From (10) and (57)

$$
\frac{\mu c^{2}}{m_{0} R^{2}}=\frac{\left(m_{f} / m_{o}\right)\left(M_{s} / m_{o}\right)}{m_{f} / m_{0}+M_{s} / m_{0}} \cdot\left(\frac{\ell_{2}+L / 2}{R}\right)^{2}=\frac{(1-\tau) \psi}{(1-\tau+\psi)}\left(\delta_{2}+\frac{\delta}{2}\right)^{2}
$$

so that

$$
\frac{\mu^{\prime} c^{2}}{m_{0} R^{2}}=-\frac{\psi^{2}}{(1-\tau+\psi)^{2}}\left(\delta_{2}+\frac{\delta}{2}\right)^{2}
$$


(20) yields

$$
\bar{I}^{\prime}=\bar{I}_{f}^{\prime}+\frac{\mu^{\prime} c^{2}}{m_{0} R^{2}}+\frac{2 \mu^{\prime} c c^{\prime}}{m_{0} R^{2}}
$$

We thus have, from (62), (54), (61) and (59) that

$$
\overline{\mathrm{I}}^{\prime}=-\frac{\delta^{2}}{12}-\frac{\tau}{2}-\frac{\psi^{2}}{(\psi+1-\tau)^{2}}\left(\delta_{2}+\frac{\delta}{2}\right)^{2}
$$

Similarly, (21) and (56) give

$$
\overline{\mathrm{J}}^{\prime}=-\overline{\mathrm{J}}_{\mathrm{f}}^{\prime}=-\tau
$$

Now (see Fig. 2),

$$
\frac{\ell_{\mathrm{e}}}{\mathrm{R}}=\frac{\ell_{1}}{\mathrm{R}}+\frac{\mathrm{L}}{2 \cdot \mathrm{R}}=\delta_{1}+\frac{\delta}{2}
$$

and (52) leads to 


$$
\frac{m^{\prime}}{m_{f 0}}=\frac{m_{f}^{\prime}}{m_{f 0}}=\left(\frac{m_{f}}{m_{f 0}}\right)^{\prime}=-1
$$

Hence,

$$
\frac{m^{\prime}}{m_{\mathfrak{f}}}\left[\left(\frac{\ell_{c}^{2}}{R^{2}}\right)+\frac{\beta^{2}}{4}\right]=-\left[\left(\delta_{1}+\frac{\delta}{2}\right)^{2}+\frac{\beta^{2}}{4}\right]
$$

and (35), (63) and (67) yield

$$
\phi(\tau)=\left(\delta_{1}^{2}+\frac{\delta^{2}}{6}+\delta_{1} \delta+\frac{\beta^{2}}{4}\right)-\frac{\tau}{2}-\frac{\psi^{2}}{(\psi+1-\tau)^{2}}\left(\delta_{2}+\frac{\delta}{2}\right)^{2}
$$

In the same way, (36), (64) and (66) give

$$
\theta(\tau)=-\tau+\frac{\beta^{2}}{2}
$$

\subsubsection{Spin Rate Analysis}

From (69), the function $\theta(\tau)$ is a linear function of $\tau$; it has a slope of -1 and is positive and equal to $\beta^{2} / 2$ at $\tau=0$, and is equal to $\beta^{2} / 2-1$ at $\tau=1$. Fig. 3 shows $\theta$ for various values of $\boldsymbol{\beta}$. 


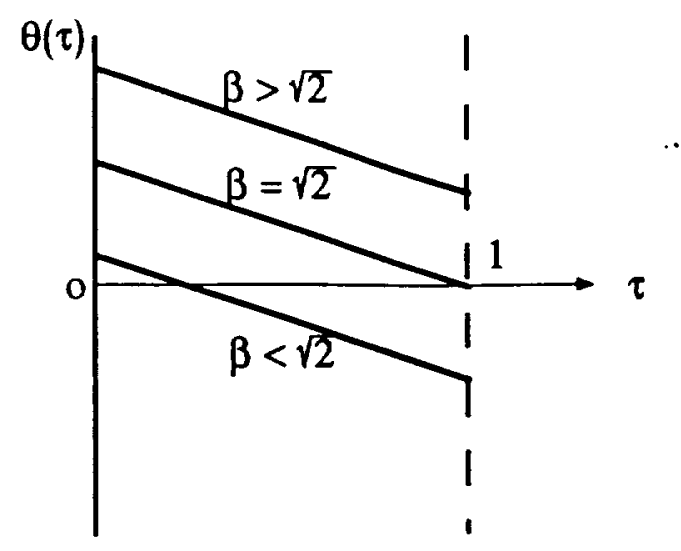

Fig. 5.3 The function $\theta$ for centrifugal burn

We conclude from the figure that the spin rate always decreases at the beginning of the burn. If $\beta \geq \sqrt{2}$, the spin rate continues to decrease all the way to burnout. For $\beta<\sqrt{2}$, the spin rate bottoms out during the burn, and then increases during the later phases of the burn. The turning point for this latter case occurs at

$$
\theta=-\tau+\beta^{2} / 2=0
$$

That is, at

$$
\tau=\beta^{2} / 2
$$


This result is consistent with, and augments that obtained for the cylinder, for which $\beta=1$ [ see (4.57) and Fig. 4.8]. The same result is validated by Fig. 4, obtained by numerical integration of (40).

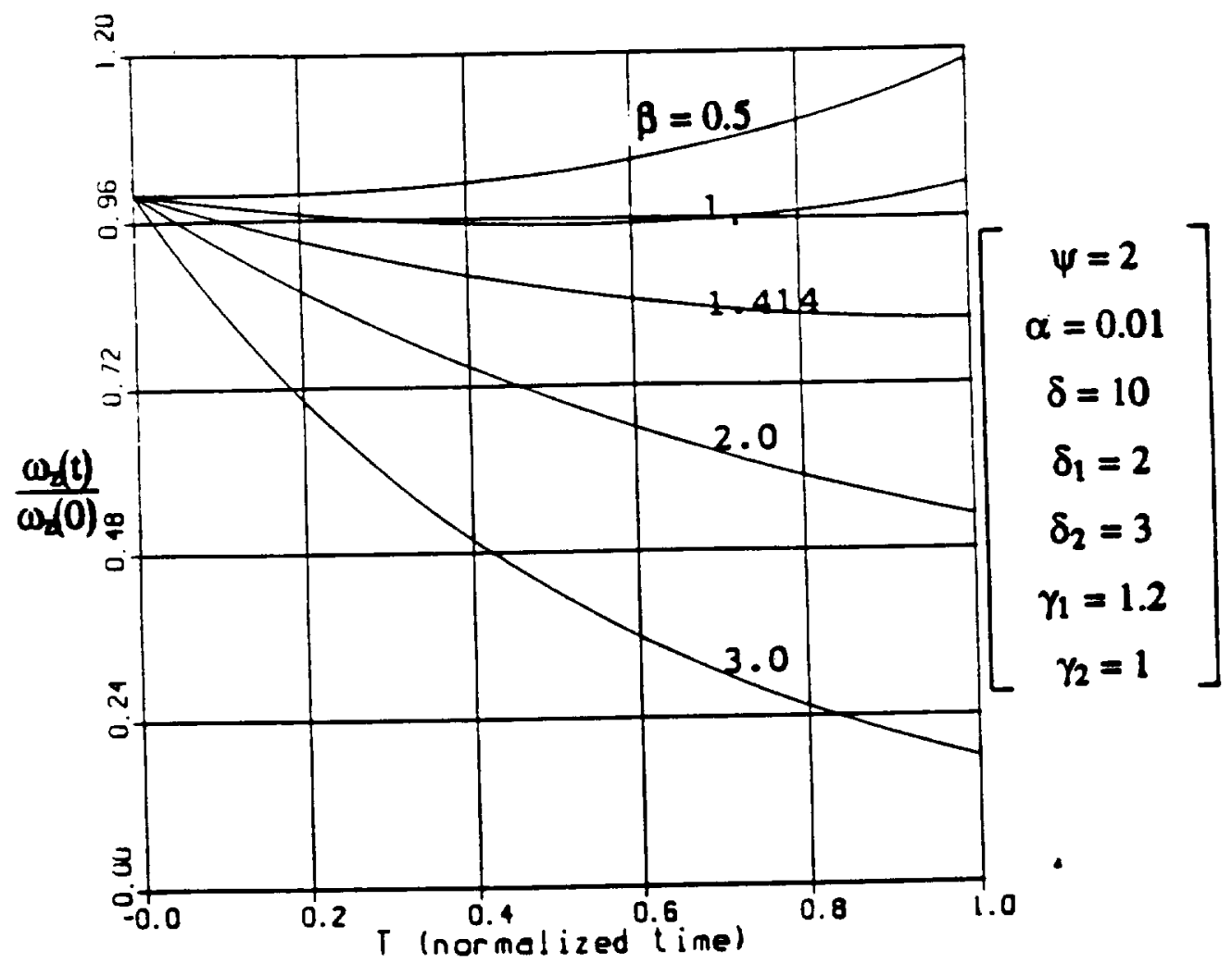

Fig. 5.4 Centrifugal burn : the effect of expansion ratio $\beta$ on spin rate

\subsubsection{Transverse Rate Analysis}

From (68), the slope of the function $\phi(\tau)$ is

$$
\phi^{\prime}=-\frac{1}{2}-\frac{2 \psi^{2}}{(\psi+1-\tau)^{3}}\left(\delta_{2}+\frac{\delta}{2}\right)^{2}
$$

and remains negative throughout the burn. 
At ignition $(\tau=0)$,

$$
\phi_{0}=\left(\delta_{1}^{2}+\frac{\delta^{2}}{6}+\delta_{1} \delta+\frac{\beta^{2}}{4}\right)-\frac{\psi^{2}}{(\psi+1)^{2}}\left(\delta_{2}+\frac{\delta}{2}\right)^{2}
$$

and at burnout $(\tau=1)$,

$$
\phi_{1}=\left(\delta_{1}^{2}+\frac{\delta^{2}}{6}+\delta_{1} \delta+\frac{\beta^{2}}{4}\right)-\frac{1}{2}-\left(\delta_{2}+\frac{\delta}{2}\right)^{2}
$$

It is clear from (72) and (73) that $\phi_{0}>\phi_{1}$, and that it is possible for any of these quantities to be negative or positive. These facts, coupled with the fact that $\phi$ is negative, mean that the three scenarios depicted in Fig. 5 are possible, depending on the values assigned to the parameters.

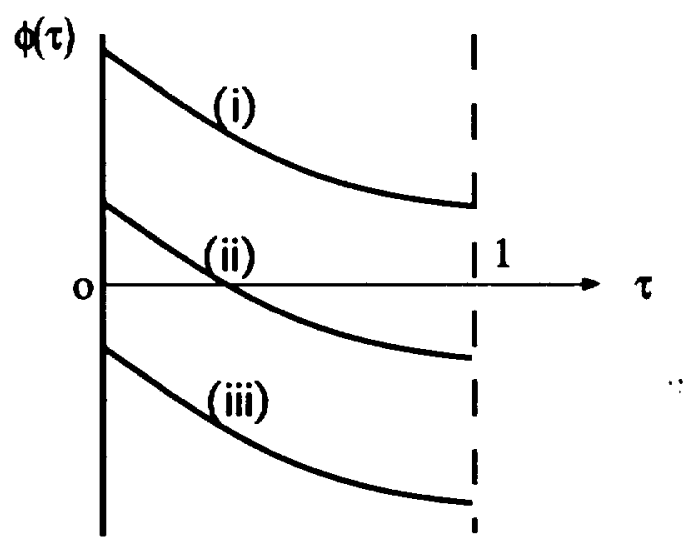

Fig. 5.5 The function $\phi$ for centrifugal burn 
We conclude from Fig. 5 that the magnitude of the transverse angular velocity for centrifugal burn can (i) decrease from ignition to burnout, (ii) decrease initially, then diverge later, or (iii) increase from ignition to burnout, depending on system parameters. Factors such as the distance of the exit plane from the combustion chamber $\left(\delta_{1}\right)$, shape factor of the propellant grain $(\delta)$, location of the propellant grain $\left(\delta_{2}\right)$, nozzle expansion ratio $(\beta)$, and the relative amount of propellant in the system $(\psi)$, all affect the transverse angular velocity. The main difference between the general axisymmetric system being considered here and the variable mass cylinder considered in chapter 4 is that case (iii) in Fig. 5 does not exist for cylinders. In other words, the transverse angular velocity of a variable mass cylinder always decreases initially, while a general axisymmetric system can exhibit divergent transverse rate throughout the propellant burn. Fig. 6 shows the transverse angular velocity magnitude as obtained by numerical integration of (39).

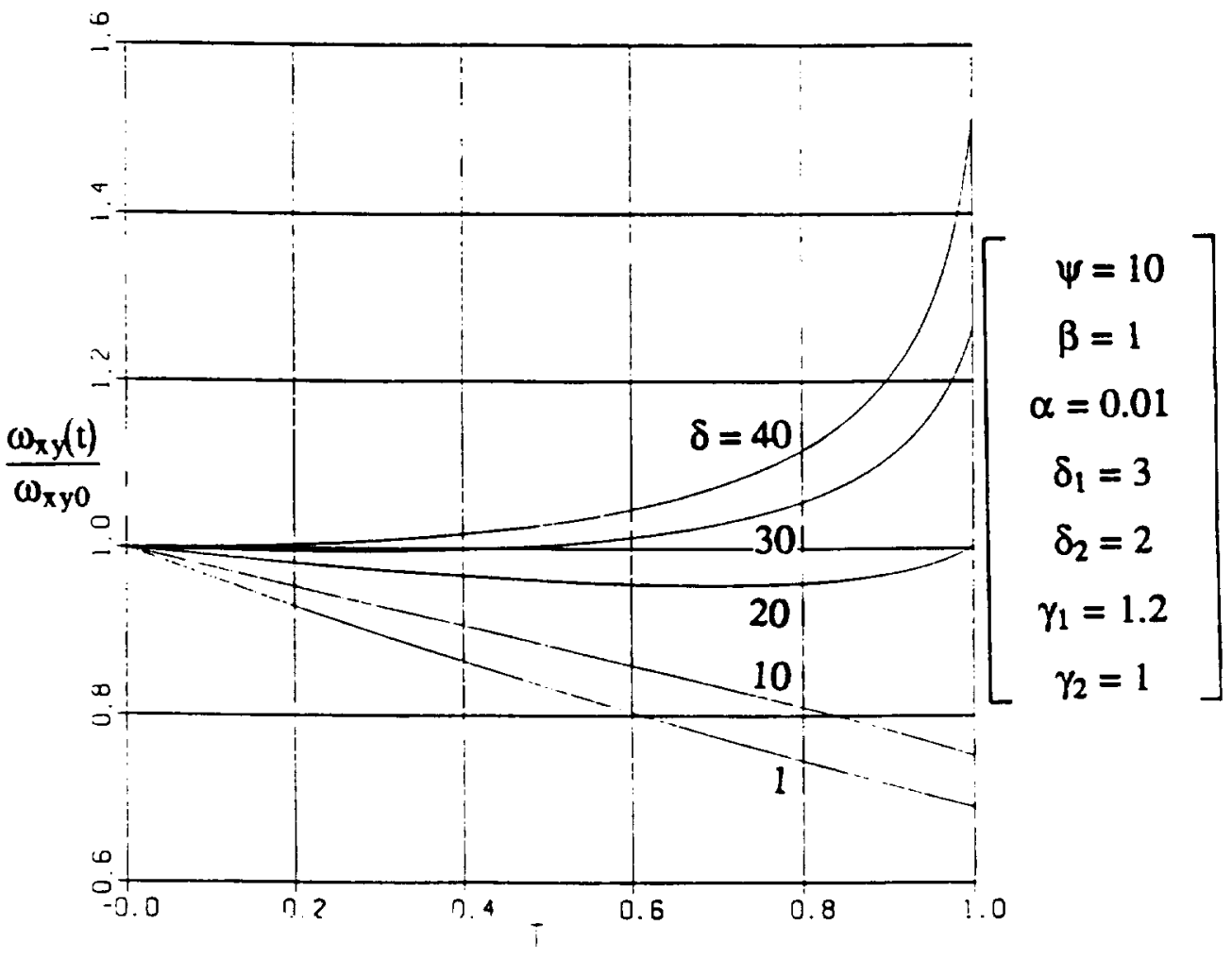

Fig. 5.6 Centrifugal burn : the effect of various $\delta$ on cross-spin 


\section{$5.4 \quad$ Uniform Burn}

We recall that in uniform burn, the dimensions of $\mathrm{F}$ remain unchanged, while its density decreases continuously with time - see Fig. 7 . In this case, $m_{f}$ has the same expression as $m_{\mathrm{fo}}$ in (42). This expression and (44) lead to

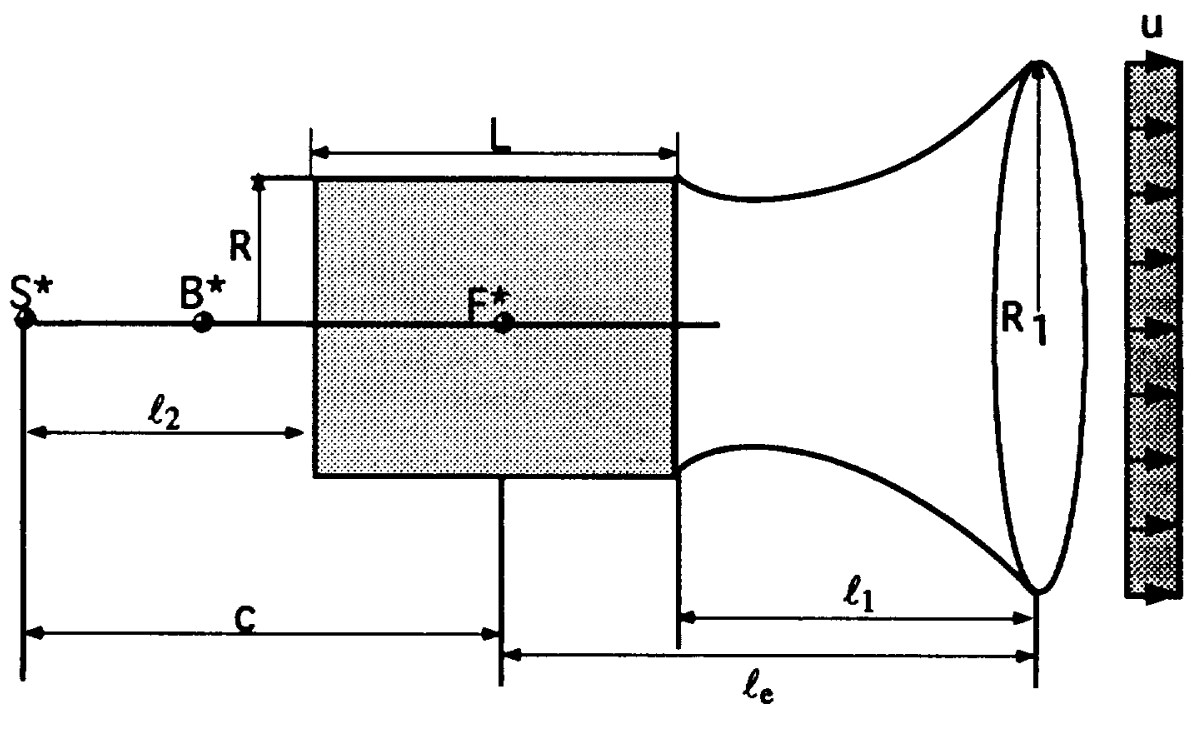

Fig. 5.7 Uniform Burn

$$
\frac{\mathrm{dm}_{\mathrm{f}}}{\mathrm{dt}}=\dot{\rho}_{\mathrm{f}} \pi \mathrm{LR} \mathrm{R}^{2}=-\rho \pi \mathrm{R}_{1}^{2} \mathrm{u}
$$

Hence

$$
\int_{\rho_{f 0}}^{\rho_{f}} d\left(\rho_{f}\right)=-\rho \frac{R_{1}^{2} u}{R^{2} L} \int_{0}^{t} d t
$$


so

$$
\rho_{f}=\rho_{f 0}-\rho \frac{R_{1}^{2} u}{R^{2} L} t
$$

From (74) and (76)

$$
\begin{gathered}
m_{f}=\rho_{f 0} \pi R^{2} L-\rho \pi R_{1}^{2} u t \\
\frac{m_{f}}{m_{f 0}}=\frac{\rho_{f 0} \pi R^{2} L-\rho \pi R_{1}^{2} u t}{\rho_{f 0} \pi L R^{2}}=1-\alpha t=1-\tau
\end{gathered}
$$

and so,

$$
\bar{I}_{f}=\frac{I_{f}}{m_{n} R^{2}}=\frac{m_{f}}{m_{o}}\left[\frac{R^{2}}{4 R^{2}}+\frac{L^{2}}{12 R^{2}}\right]=(1-\tau)\left(\frac{1}{4}+\frac{\delta^{2}}{12}\right)
$$

from which we have

$$
\begin{gathered}
\overline{\mathrm{I}}_{\mathrm{f}}^{\prime}=-\frac{1}{4}-\frac{\delta^{2}}{12} \\
\overrightarrow{\mathrm{J}}_{\mathrm{f}}=\frac{\mathrm{J}_{\mathrm{f}}}{\mathrm{m}_{\mathrm{o}} \mathrm{R}^{2}}=\frac{\mathrm{m}_{\mathrm{f}}}{\mathrm{m}_{\mathrm{o}}} \frac{\mathrm{R}^{2}}{2 \mathrm{R}^{2}}=\frac{1-\tau}{2}
\end{gathered}
$$


and

$$
\bar{J}_{\mathrm{f}}^{\prime}=-\frac{1}{2}
$$

As before (see Fig. 7),

$$
\mathrm{c}=\ell_{2}+\mathrm{L} / 2
$$

$$
c^{\prime}=0
$$

and

$$
2 \mu c c^{\prime}=0
$$

From (10) and (83)

$$
\frac{\mu c^{2}}{m_{0} R^{2}}=\frac{\left(m_{f} / m_{0}\right)\left(M_{s} / m_{0}\right)}{m_{f} / m_{0}+M_{s} / m_{0}} \cdot\left(\frac{\ell_{2}+L / 2}{R}\right)^{2}=\frac{(1-\tau) \psi}{(1-\tau+\psi)}\left(\delta_{2}+\frac{\delta}{2}\right)^{2}
$$

and

$$
\frac{\mu^{\prime} c^{2}}{m_{0} R^{2}}=-\frac{\psi^{2}}{(1-\tau+\psi)^{2}}\left(\delta_{2}+\frac{\delta}{2}\right)^{2}
$$

We thus have, from (62), (80), (85) and (87) that 


$$
\overline{\mathrm{I}}^{\prime}=-\frac{\delta^{2}}{12}-\frac{\tau}{2}-\frac{\psi^{2}}{(\psi+1-\tau)^{2}}\left(\delta_{2}+\frac{\delta}{2}\right)^{2}
$$

Similarly, (21) and (82) give

$$
\bar{J}^{\prime}=-\bar{J}_{f}^{\prime}=-1 / 2
$$

Now (see Fig. 7),

$$
\frac{\ell_{\mathrm{e}}}{\mathrm{R}}=\frac{\ell_{1}}{\mathrm{R}}+\frac{\mathrm{L}}{2 \cdot \mathrm{R}}=\delta_{1}+\frac{\delta}{2}
$$

and (78) leads to

$$
\frac{m^{\prime}}{m_{f 0}}=\frac{m_{f}^{\prime}}{m_{f 0}}=\left(\frac{m_{f}}{m_{f 0}}\right)^{\prime}=-1
$$

Hence,

$$
\frac{m^{\prime}}{m_{f 0}}\left[\left(\frac{\ell_{e}^{2}}{R^{2}}\right)+\frac{\beta^{2}}{4}\right]=-\left[\left(\delta_{1}+\frac{\delta}{2}\right)^{2}+\frac{\beta^{2}}{4}\right]
$$

and (35), (88) and (92) yield

$$
\phi(\tau)=\left(\delta_{1}^{2}+\frac{\delta^{2}}{6}+\delta_{1} \delta+\frac{\beta^{2}}{4}\right)-\frac{1}{4}-\frac{\psi^{2}}{(\psi+1-\tau)^{2}}\left(\delta_{2}+\frac{\delta}{2}\right)^{2}
$$

In the same way, (36), (89) and (91) give 


$$
\theta(\tau)=-\frac{1}{2}+\frac{\beta^{2}}{2}
$$

\subsubsection{Spin Rate Analysis}

From (94), the function $\theta(\tau)$ is a constant. Fig. 8 shows $\theta$ for various values of $\boldsymbol{\beta}$.

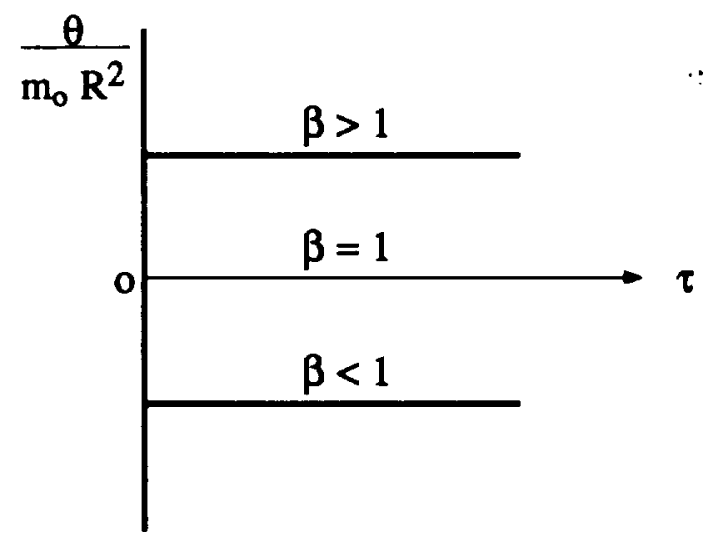

Fig. 5.8 The function $\theta$ for uniform burn

If $\beta>1$, the spin continues to decrease all the way to burnout. For $\beta=1$, the spin rate always equals its initial value. For $\beta<1$, the spin rate increases from the beginning to the end of the burn. The same result is validated by Fig. 9 , obtained by numerical integration of $(40)$ 


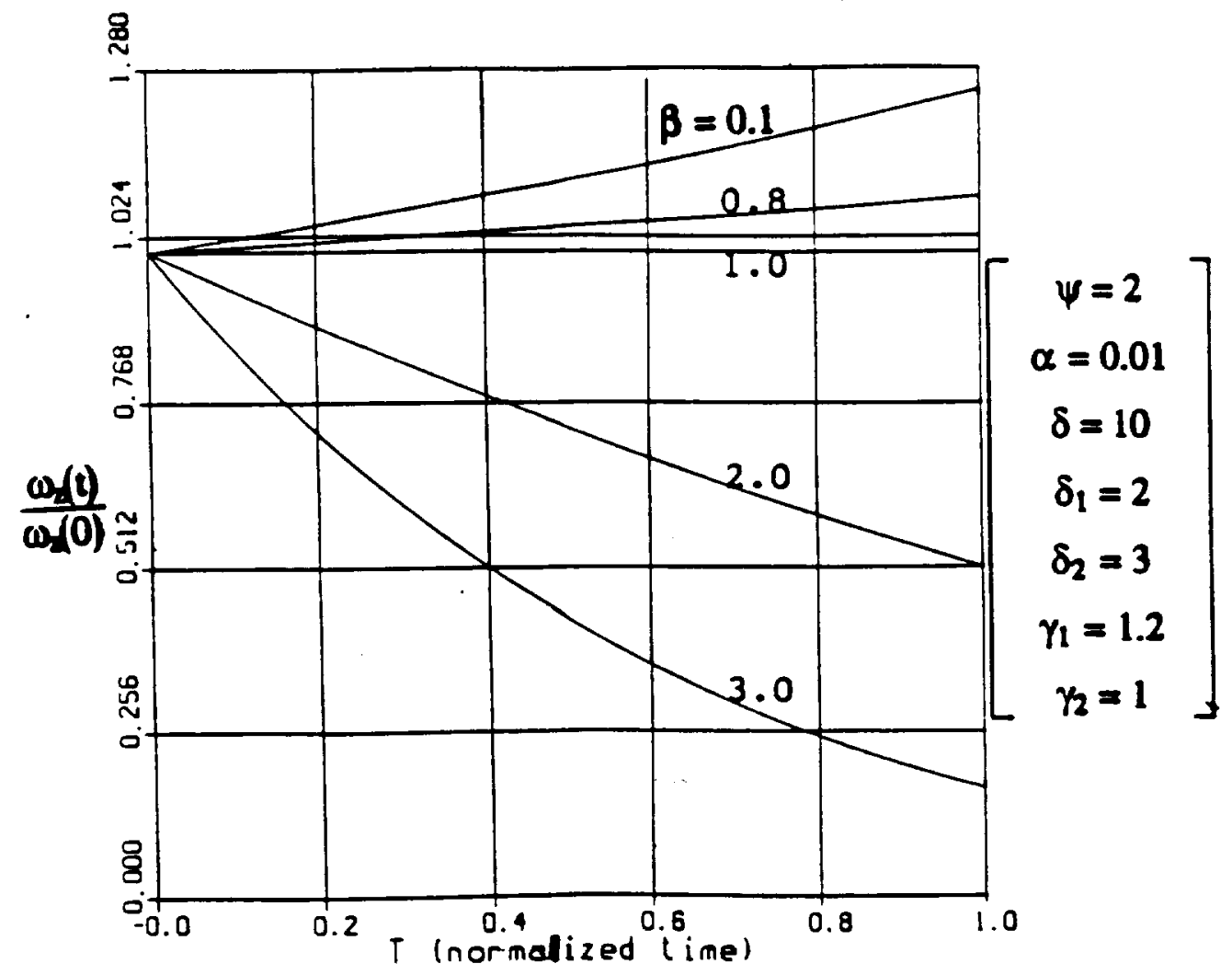

Fig. 5.9 Uniform Burn : the effect of expansion ratio $\beta$ on spin rate

\subsubsection{Transverse Rate Analysis}

From (93), the slope of the function $\phi(\tau)$ is

$$
\phi^{\prime}=-\frac{2 \psi^{2}}{(\psi+1-\tau)^{3}}\left(\delta_{2}+\frac{\delta}{2}\right)^{2}
$$

and stays negative throughout the burn. At ignition $(\tau=0)$,

$$
\phi_{0}=\left(\delta_{1}^{2}+\frac{\delta^{2}}{6}+\delta_{1} \delta+\frac{\beta^{2}}{4}\right)-\frac{1}{4}-\frac{\psi^{2}}{(\psi+1)^{2}}\left(\delta_{2}+\frac{\delta}{2}\right)^{2}
$$


and at burnout $(\tau=1)$,

$$
\phi_{1}=\left(\delta_{1}^{2}+\frac{\delta^{2}}{6}+\delta_{1} \delta+\frac{\beta^{2}}{4}\right)-\frac{1}{4}-\left(\delta_{2}+\frac{\delta}{2}\right)^{2}
$$

Hence $\phi_{0}>\phi_{1}$, always, and either of these quantities can be negative or positive. Since we also have that $\phi$ is always negative, we have the same scenario obtained for centrifugal burn. Numerical simulation results provided in Fig. 10 below confirm these inferences.

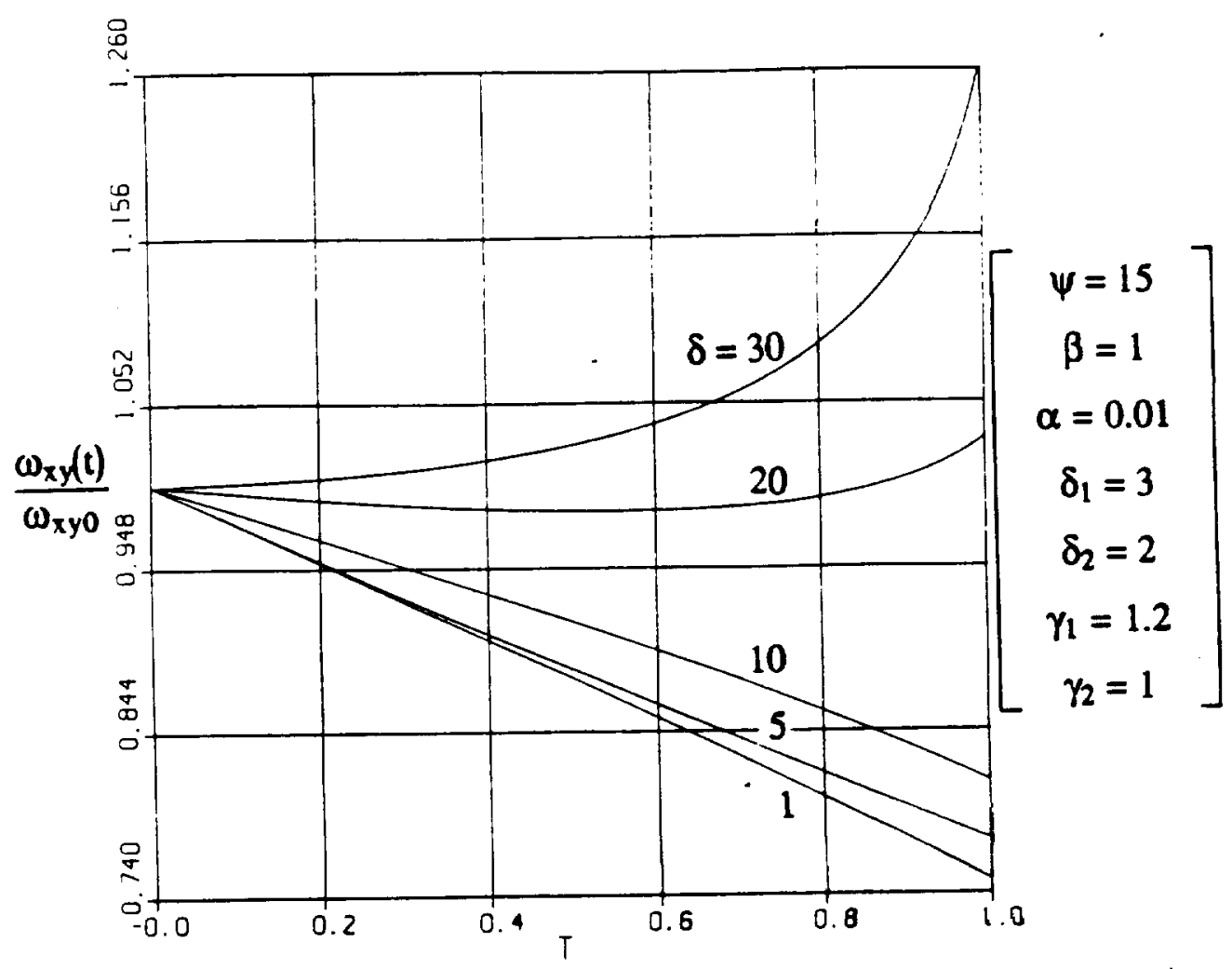

Fig. 5.10 Uniform Burn : the effect of $\delta$ on cross-spin 


\section{CHAPTER 6}

\section{FORCED MOTION OF AXISYMMETRIC SYSTEMS}

All the development of the last two chapters have been restricted to systems subjected to zero external torque. The only forcing function present has been a perfectly axial thrust, whose line of action passes through the system mass center at all times, and thus produces no couple on the system. Great pains are taken in the design of real rockets to minimize thrust misalignments. Yet, it is unrealistic to expect that the system mass center, which generally moves during rocket burn, will remain positioned on the line of action of the resultant thrust vector throughout the propellant burn. Thus, torques due to thrust misalignment are often inevitable. There are also other potential sources of extraneous torques on a rocket system in flight, including aerodynamic and gravitational moments. For this reason, we will, in this chapter, develop relationships that describe the effects of externally applied moments on the attitude behavior of the same axisymmetric variable mass system that was studied in the previous chapter. 


\subsection{Equations of Attitude Motion}

The equations of forced attitude motion of the system of Fig. 5.1 are really the same as (5.17)-(5.19), except that the right hand side of each of these equation is non-zero.

Thus, we have

$$
\begin{gathered}
I \dot{\omega}_{\mathrm{x}}+(\mathrm{J}-\mathrm{I}) \omega_{\mathrm{y}} \omega_{\mathrm{z}}+\left[\dot{\mathrm{I}}-\dot{\mathrm{m}}_{\mathrm{f}}\left(\ell_{\mathrm{e}}^{2}+\mathrm{R}_{\mathrm{l}}^{2} / 4\right)\right] \omega_{\mathrm{x}}=\mathrm{M}_{\mathrm{x}} \\
\mathrm{I} \dot{\omega}_{\mathrm{y}}-(\mathrm{J}-\mathrm{I}) \omega_{\mathrm{x}} \omega_{\mathrm{z}}+\left[\dot{\mathrm{I}}-\dot{\mathrm{m}}_{\mathrm{f}}\left(\ell_{\mathrm{e}}^{2}+\mathrm{R}_{1}^{2} / 4\right)\right] \omega_{\mathrm{y}}=\mathrm{M}_{\mathrm{y}} \\
\cdot \\
\mathrm{J} \dot{\omega}_{\mathrm{z}}+\left(\dot{\mathrm{J}}_{\mathrm{f}}-\dot{\mathrm{m}}_{\mathrm{f}} \mathrm{R}_{1}^{2} / 2\right) \omega_{\mathrm{z}}=\mathrm{M}_{\mathrm{z}}
\end{gathered}
$$

The forcing functions $\mathbf{M}_{x}, \mathbf{M}_{\mathbf{y}}$ and $\mathbf{M}_{\mathbf{z}}$ are, in general, functions of time. This is so because at least the contributions from thrust misalignments vary with time since the system mass center moves relative to the system base structure.

We start the solution process by letting

$$
\lambda_{2}=\left(\dot{\mathrm{J}}_{\mathrm{f}}-\dot{\mathrm{m}}_{\mathrm{f}} \mathrm{R}_{1}^{2} / 2\right) / \mathrm{J}
$$

and

$$
\overline{\mathbf{M}}_{\mathbf{z}}=\mathbf{M}_{\mathbf{z}} / \mathbf{J}
$$


(3) then becomes

$$
\frac{d \omega_{2}}{d t}+\lambda_{2}(t) \omega_{z}=\bar{M}_{z}
$$

The general solution of (6) is

$$
\omega_{z}=\left[\omega_{z 0}+\int_{0}^{t} \bar{M}_{d}(s) \exp \left(\int_{0}^{s} \lambda_{2}(\tau) d \tau\right) d s\right] \exp \left(-\int_{0}^{t} \lambda_{2}(\tau) d \tau\right)
$$

This solution can be thought of as the sum of two components : a first term that represents the homogenous part due to initial condition, and a second term - the particular solution that is contributed by the forcing function. If $\mathrm{J}, \dot{\mathrm{J}}_{\mathrm{f}}, \dot{\mathrm{m}}_{\mathrm{f}}$ and $\mathrm{M}_{\mathrm{Z}}$ are known functions of time, and the initial spin rate is known, (7) determines uniquely the spin rate $\omega_{z}$ of the body S. Note that $\omega_{z}$ depends only on $\lambda_{2}$ and $\bar{M}_{z}$, and is independent of $M_{x}$ and $M_{y}$. This is true because of our assumption of axisymmetry.

We are now ready to investigate the transverse angular velocity components of the system. To this end, we introduce the following complex quantities :

$$
\omega_{x y}=\omega_{x}+j \omega_{y}
$$

$$
M_{\mathbf{x y}}=M_{\mathbf{x}}+\mathbf{j} \mathbf{M}_{\mathbf{y}}
$$


where

$$
j=\sqrt{-1}
$$

We also define

$$
\begin{gathered}
\lambda_{1}=\left[\dot{\mathrm{I}}-\dot{\mathrm{m}}_{\mathrm{f}}\left(\ell_{\mathrm{e}}^{2}+\mathrm{R}_{1}^{2} / 4\right)\right] / \mathrm{I} \\
\sigma=(\mathrm{J}-\mathrm{I}) / \mathrm{I}
\end{gathered}
$$

and

$$
\bar{M}_{x y}=M_{x y} / I
$$

Adding $\mathrm{j}$ times (2) to (1), we obtain

$$
I \dot{\omega}_{x y}-j(J-I) \omega_{z}\left(j \omega_{y}+\omega_{x}\right)+\lambda_{1} \omega_{x y}=M_{x y}
$$

or 


$$
\frac{d \omega_{x y}}{d t}+P_{2}(t) \omega_{x y}=\bar{M}_{x y}
$$

where

$$
P_{2}(t)=\lambda_{1}-\sigma \omega_{2}
$$

The solution to (16) is

$$
\omega_{x y}=\left[\omega_{x y 0}+\int_{0}^{t} \bar{M}_{x y}(s) \exp \left(\int_{0}^{s} P_{2}(\tau) d \tau\right) d s\right] \exp \left(-\int_{0}^{t} P_{2}(\tau) d \tau\right)
$$

or equivalently,

$$
\begin{gathered}
\omega_{\mathrm{x}}+\mathrm{j} \omega_{\mathrm{y}}=\left\{\omega_{\mathrm{x} 0}+\mathrm{j} \omega_{\mathrm{y} 0}+\int_{0}^{t}\left[\mathrm{M}_{\mathrm{x}}(\mathrm{s}) / \mathrm{I}+\mathrm{j} \mathrm{M}_{\mathrm{y}}(\mathrm{s}) / \mathrm{I}\right]\right. \\
\{\exp [\Gamma(\mathrm{s})]\}[\cos \Theta(s)-\mathrm{j} \sin \Theta(s)] \mathrm{ds}\rangle\langle\exp [-\Gamma(s)]\}[\cos \Theta(\mathrm{t})+\mathrm{j} \sin \Theta(\mathrm{t})]
\end{gathered}
$$

where

$$
\Gamma(t)=\int_{0}^{t} \lambda_{1} d \tau
$$

and 


$$
\Theta(t)=\int_{0}^{t}\left(\sigma \omega_{2}\right) d \tau
$$

The functions $\Gamma(t)$ and $\Theta(t)$, which have fundamentally different properties, govern the character of the transverse motion of the variable mass system under consideration. $\Gamma(t)$ determines the amplitude of the angular motion and $\Theta(t)$ affects motion frequency. Explicit formula for $\omega_{x}$ and $\omega_{y}$ can be obtained from (18) by separating the real and imaginary parts of the expression; they are :

$$
\begin{gathered}
\omega_{x}(t)=e^{-\Gamma(t)}\left[\omega_{x 0} \cos \Theta(t)-\omega_{y 0} \sin \Theta(t)\right]+ \\
\int_{t_{0}}^{t} \frac{e^{[\Gamma(s)-\Gamma(t)]}}{I}\left\{M_{x}(s) \cos [\Theta(s)-\Theta(t)]+M_{y}(s) \sin [\Theta(s)-\Theta(t)]\right\} d s
\end{gathered}
$$

and

$$
\begin{aligned}
& \omega_{y}(t)=e^{-\Gamma(t)}\left[\omega_{x 0} \sin \Theta(t)+\omega_{y 0} \cos \Theta(t)\right]+ \\
& \int_{t_{0}}^{t} \frac{e^{[\Gamma(s)-\Gamma(t)]}}{I}\left\{M_{y}(s) \cos \{\Theta(s)-\Theta(t)]-M_{x}(s) \sin [Q(s)-Q(t)]\right\} d s
\end{aligned}
$$

We observe that in general the spin torque, $M_{\mathbf{z}}$ has no effect on the transverse angular velocity, nor is the spin rate affected by any of the transverse torques. 


\subsection{Change of the Independent Variable}

We now introduce a new independent variable in order to simplify somewhat the equations of attitude motion and their solution. We exploit here, a transformation defined earlier and first proposed by Jarmolow (1957) :

$$
\Theta(t)=\int_{0}^{t} \omega_{2} \frac{(J-I)}{I} d \zeta=\int_{0}^{t} \omega_{2} \sigma d \zeta
$$

$\Theta$, has the dimension of angle (radians) and is related to the spin angle. It will be used as the new measure of time.

Note that

$$
\mathrm{d} \Theta=\omega_{\mathbf{2}} \frac{(\mathrm{J}-\mathrm{I})}{\mathrm{I}} \mathrm{dt}
$$

so

$$
\frac{d}{d t}=\omega_{2} \frac{\left(J^{*}-I^{*}\right)}{I^{*}} \frac{d}{d \Theta}
$$

The starred quantities are assumed to be expressed in terms of the new variable $\Theta$. This is indeed possible because (23) gives $\Theta$ as a function of $t$, and can be inverted to yield the 
expression of $t$ as a function of $\Theta$. Equations (1) and (2) can be re-written in terms of $\Theta$ as follows :

$\omega_{\mathrm{z}}\left(\mathrm{J}^{*}-\mathrm{I}^{*}\right) \frac{\mathrm{d} \omega_{\mathrm{x}}}{\mathrm{d} \Theta}+\left(\mathrm{J}^{*}-\mathrm{I}^{*}\right) \omega_{\mathrm{y}} \omega_{\mathrm{z}}+\left[\frac{\mathrm{d} \mathrm{I}^{*}}{\mathrm{~d} \Theta}-\frac{\mathrm{d} \mathrm{m}_{\mathrm{f}}^{*}}{\mathrm{~d} \Theta}\left(\ell_{\mathrm{e}}^{* 2}+\mathrm{R}_{1}^{2} / 4\right)\right] \frac{\left(\mathrm{I}_{\mathrm{z}}^{*}-\mathrm{I}^{*}\right)}{\mathrm{I}^{*}} \omega_{\mathrm{z}} \omega_{\mathrm{x}}=\mathrm{M}_{\mathrm{x}}^{*}$

$\omega_{z}\left(J^{*}-I^{*}\right) \frac{d \omega_{y}}{d \Theta}-\left(J^{*}-I^{*}\right) \omega_{x} \omega_{z}+\left[\frac{d I^{*}}{d \Theta}-\frac{d m_{f}^{*}}{d \Theta}\left(\ell_{e}^{* 2}+R_{1}^{2} / 4\right)\right] \frac{\left(J^{*}-I^{*}\right)}{I^{*}} \omega_{z} \omega_{y}=M_{y}^{*}$

Dividing through by $\left(\mathrm{J}^{*}-\mathrm{I}^{*}\right) \omega_{2}$, one obtains

$$
\frac{d \omega_{\mathrm{x}}}{\mathrm{d} \Theta}+\omega_{\mathrm{y}}+\left[\frac{\mathrm{dI}}{\mathrm{d} \Theta}-\frac{\mathrm{d} \mathrm{m}_{\mathrm{f}}^{*}}{\mathrm{~d} \Theta}\left(\ell_{\mathrm{e}}^{* 2}+\mathrm{R}_{1}^{2} / 4\right)\right] \frac{\omega_{\mathrm{x}}}{\mathrm{I}^{*}}=\frac{\mathrm{M}_{\mathrm{x}}^{*}}{\omega_{\mathrm{z}}\left(\mathrm{J}^{*}-\mathrm{I}^{*}\right)}
$$

and

$$
\frac{d \omega_{y}}{d \theta}-\omega_{x}+\left[\frac{d I^{*}}{d \Theta}-\frac{d m_{f}^{*}}{d \Theta}\left(\ell_{e}^{* 2}+R_{1}^{2} / 4\right)\right] \frac{\omega_{y}}{I^{*}}=\frac{M_{y}^{*}}{\omega_{z}\left(J^{*}-I^{*}\right)}
$$

Then, multiplying (29) by $\mathrm{j}$ and adding to (28), we have

$$
\frac{d \omega_{x y}}{d \Theta}+\left(\lambda_{1}^{*}-j\right) \omega_{x y}=M_{x y}^{*}
$$


where

$$
\begin{gathered}
\omega_{x y}(\Theta)=\omega_{x}(\Theta)+j \omega_{y}(\Theta) \\
\lambda_{1}^{*}=\left[\frac{d I^{*}}{d \Theta}-\frac{d m_{f}^{*}}{d \Theta}\left(l_{e}^{* 2}+R_{1}^{2} / 4\right)\right] /_{I^{*}}
\end{gathered}
$$

and

$$
M_{x y}=\frac{M_{x}^{*}+j M_{y}^{*}}{\omega_{z}\left(J^{*}-I^{*}\right)}
$$

If we define $\Gamma^{*}(\Theta)$ as

$$
\Gamma^{*}(\Theta)=\int_{0}^{\Theta} \lambda_{1}^{*} d \tau
$$

then the solution of (30) for $\omega_{\mathrm{x}}$ and $\omega_{\mathrm{y}}$ in terms of the new variable $\Theta$ can be written explicitly as

$$
\begin{gathered}
\omega_{\mathrm{x}}(\Theta)=\left(\omega_{\mathrm{x} 0} \cos \Theta-\omega_{\mathrm{y} 0} \sin \Theta\right) \mathrm{e}^{-\Gamma^{*}(\Theta)}+ \\
\int_{0}^{\Theta} \frac{\mathrm{e}^{\left[\Gamma^{*}(\mathrm{~s})-\Gamma^{*}(\Theta)\right]}}{\omega_{\mathrm{z}}\left(\mathrm{J}^{*}-\mathrm{I}^{*}\right)}\left[\mathrm{M}_{\mathrm{x}}^{*}(\mathrm{~s}) \cos (\mathrm{s}-\Theta)+\mathrm{M}_{\mathrm{y}}^{*}(\mathrm{~s}) \sin (\mathrm{s}-\Theta)\right] \mathrm{ds}
\end{gathered}
$$




$$
\begin{gathered}
\omega_{y}(\Theta)=\left[\omega_{x 0} \sin \Theta+\omega_{y 0} \cos \Theta\right] \mathrm{e}^{-\Gamma^{*}(\Theta)}+ \\
\int_{0}^{\Theta} \frac{\mathrm{e}^{\left[\Gamma^{*}(\mathrm{~s})-\Gamma^{*}(\Theta)\right]}}{\omega_{z}\left(\mathrm{~J}^{*}-\mathrm{I}^{*}\right)}\left[\mathrm{M}_{\mathrm{y}}^{*}(\mathrm{~s}) \cos (\mathrm{s}-\Theta)-\mathbf{M}_{\mathrm{x}}^{*}(\mathrm{~s}) \sin (\mathrm{s}-\Theta)\right] \mathrm{ds}
\end{gathered}
$$

In order to obtain explicit expressions for the known functions such as I, J, $M_{\mathbf{x}}$ and $M_{y}$, the function $t(\Theta)$ must be determined. This may or may not be a trivial task. The integrand of (23) is a rational function of time whose integral may not have a closed form analytical expression. However, a numerical solution is always possible, so we can be sure that the complete solution sketched above can always be obtained by numerical means - at least.

\subsection{Special Case - Rigid Body Motion}

The above solution can be used to investigate the artitude motion of an axisymmetric rigid body simply by setting

$$
\frac{d^{*}}{d \Theta}=\frac{d^{*}}{d \Theta}=\frac{d m_{f}}{d \Theta}=0
$$

(32) and (34) then yield

$$
\Gamma^{*}(\theta)=0
$$

and (35) and (36) simplify as follows 


$$
\begin{gathered}
\omega_{\mathrm{x}}(\Theta)=\left(\omega_{\mathrm{x} 0} \cos \Theta-\omega_{\mathrm{y} 0} \sin \Theta\right)+ \\
\int_{0}^{\Theta} \frac{1}{\omega_{\mathrm{z}}\left(\mathrm{J}^{*}-\mathrm{I}^{*}\right)}\left[\mathrm{M}_{\mathrm{x}}^{*}(\mathrm{~s}) \cos (\mathrm{s}-\Theta)+\mathrm{M}_{\mathrm{y}}^{*}(\mathrm{~s}) \sin (\mathrm{s}-\Theta)\right] \mathrm{ds}
\end{gathered}
$$

$$
\omega_{y}(\Theta)=\left[\omega_{x 0} \sin \Theta+\omega_{y 0} \cos \Theta\right]+
$$

$$
\int_{0}^{\Theta} \frac{1}{\omega_{z}\left(J^{*}-I^{*}\right)}\left[M_{y}^{*}(s) \cos (s-\Theta)-M_{x}^{*}(s) \sin (s-\Theta)\right] d s
$$

In this form, the physical significance of the various parameters of the problem is not so obvious. Simpler and more revealing expressions for $\omega_{\mathrm{x}}$ and $\omega_{\mathrm{y}}$ are obtained for the case of constant body-fixed torques. For instance, if we take $M_{x}$ and $M_{y}$ to be constants, and $\mathbf{M}_{\mathbf{z}}=0,(39)$ and (40) further simplify to

$$
\begin{gathered}
\omega_{x}(\Theta)=\left[\omega_{x 0} \cos (\Theta)-\omega_{y 0} \sin (\Theta)\right]+ \\
\frac{1}{\left(J^{*}-I^{*}\right) \omega_{z 0}}\left\{M_{x}^{*} \sin (\Theta)-M_{y}^{*}[1-\cos (\Theta)]\right\} \\
\omega_{y}(\Theta)=\left[\omega_{y 0} \cos (\Theta)+\omega_{x 0} \sin (\Theta)\right]+ \\
\frac{1}{\left(J^{*}-I^{*}\right) \omega_{z 0}}\left\{M_{y}^{*} \sin (\Theta)+M_{x}^{*}[1-\cos (\Theta)]\right\}
\end{gathered}
$$

where 


$$
\Theta(t)=\frac{J-I}{I} \omega_{20} t
$$

We note here that the spin rate remains constant at its initial value, as can be seen from (4), (5) and (7). (41) and (42) indicate that transverse velocities increase with transverse torques and decrease with the inertia difference, $\mathrm{J}^{*}-\mathrm{I}^{*}$, and initial spin rate.

Spinning a rigid body about one of its principal axes provides the so-called spin rigidity or gyroscopic stiffness; that is, a resistance to external disturbances during motion. This is the well known advantage of spin stabilization. In a spin stabilized system, the transverse angular rates $\omega_{\mathrm{x}}$ and $\omega_{\mathrm{y}}$ are usually the result of unforeseen disturbance, and are generally undesirable. If $\mathbf{M}_{\mathbf{x}}$ and $\mathbf{M}_{\mathbf{y}}$ are viewed as constants, body-fixed disturbance torques in (41) and (42) above, then, these equations show clearly that high spin rates will reduce the effects of the disturbance torques on vehicle motion. This indicates that the nominal spin rate of a spin stabilized vehicle should be given the highest value compatible with the vehicle's structural integrity. A fact that agrees with physical intuition. It can thus be said that as the spin rate increases, the stability of a given system increases as measured by the reduction in angular rates introduced by a given disturbance.

For a single spinning body, (41) and (42) also show that gyroscopic stiffness depends in a crucial way on the system inertia property, $\mathrm{J}^{*}-\mathrm{I}^{*}$, or the difference between the spin and the transverse moment of inertia of the body. Spin rigidity vanishes as the inertia difference tends to zero. In other words, bodies with spherical symmetry can have no gyroscopic stiffness, irrespective of the value the spin rate. On the other hand, heavily prolate or oblate bodies are seen to be quite stable in spin. 


\section{CHAPTER 7}

\section{CONCLUSION}

The study presented in this report deals with the dynamic behavior of spinning bodies that lose mass while in motion. The study began with the determination of the complete equations of such systems using one of the most powerful methods of analytical dynamics - Kane's formalism. The resulting equations are then applied to the study of the attitude dynamics of space-based variable mass systems using mathematical models that vary from a simple cylinder to general axisymmetric systems. The emphasis of these efforts was on information extraction. Special mathematical techniques were used to extract as much information as possible about the motion of the system, without actually attempting a full solution of the equations of motion.

In the first example presented, a variable mass rocket system is represented by a simple cylinder with four possible mass loss scenarios. Results obtained indicate that such a system can fly stably in a torque free environment if the ratio of the radius to the length is small. Large and short cylindrical vehicles can have stability problems, especially if they are subjected to radial burn. This study goes far beyond previous work in this area by clearly identifying the exact inertia conditions and the precise stages of vehicle motion at 
which the character of motion begins to change. For cases where there is stability issue, limiting values of motion parameters are determined.

Attention was next turned to a slightly more complicated model - a general axisymmetric variable mass system. Although important differences were found in the details of the motion of the general axisymmetric system as compared to that of the simple cylinder, the main stability results did not change. It was found in this second example, that in addition to the shape factor of the system propellant, the location of the propellant grain within the system as well as the nozzle expansion ratio all have significant effects on the attitude motion of a rocket.

In the last section of this study, the combined effect of mass loss and external transverse torques on system motion is examined, and solutions to the equations of motion in this case are presented. Overall, this study augments considerably, previous knowledge of the dynamics of variable mass systems and gives great insight into the effects of mass variation on the attitude behavior of spinning bodies.

This study shows precisely how the system's attitude response is tied to such system parameters as nozzle geometry, combustion chamber geometry, location and relative mass of the propellant. In this sense, the study will be of great use to designers in that it indicates what must be done to avoid motion instability of the type witnessed in the 80 's on Star 48 propelled space vehicles. 


\section{REFERENCES AND BIBLIOGRAPHY}

1. Armstrong, H. L. (1965). "Rotation And Angular Acceleration." American Journal Of Physics 33: 507.

2. Armstrong, H. L. (1966). "On the Form in Which Newton's Law is Expressed." American Joumal of Physics 34: 982.

3. Ball, K. J. and G. F. Osborne (1967). Space Vehicles Dynamics. Oxford. 153157.

4. Barton, M. V. (1950). "The Effect of Variation in Mass on the Dynamic Stability of Jet Propelled Missiles." Journal of the Aeronautical Sciences 17: 197-203.

5. Belknap, S. B. (1970). Yariable Mass Dypamics for Engineering Systems. Ph.D. Dissertation, University of California, Los Angeles.

6. Belknap, S. B. (1972). "A General Transport Rule for Variable Mass Dynamics." AIAA Journal 10(9): 1137-1138.

7. Bernoulli, D., (1738), Hydrodynamica, Argentorati.

8. Bottaccini, M. (1963). “An Alternate Interpretation of Newton's Second Law." AIAA Joumal 1(4): 927-928. 
9. Bottaccini, M. (1964). “Addendum: “An Alternate Interpretation of Newton's Second Law"." AIAA Joumal 2(6): 1164-1165.

10. Breuer, D. W. and W. R. Southernland (1965). "Jet damping effects: theory and experiment." Joumal of Spacecraft and Rocket 2(4): 638-639.

11. Buglia, J. J., G. R. Young, et al. (1961). Analytical Method of Approximating the Motion of a Spinning Vehicle with Variable Mass and Inertial Properties Acted Upon by Several Disturbing Parameters. NASA TR-110.

12. Buquoy, G., (1816), "Exposition d'un Nouveau Principe General de Dynamique, Paris.

13. Byrne, J. W. J. and S. Raynor (1964). "An Analysis of the Yawing Motion of a Rocket with a Varying Mass." AIAA Joumal 2(5): 962-963.

14. Cochran, J.E. and Kang, J.Y., (1991) "Nonlinear Stability Analysis of the Attitude Motion of a Spin Stabilized Upper Stage" AAS Paper 91-109, Advances in the Astronautical Sciences, V75, 345-364

15. Cohan, F. A. (1966). The Influence of Rocket Motor Geometry on Jet Damping. MS Thesis, University of Califomia, Los Angeles.

16. Copeland, J. (1982). "Work-Energy Theorem for Variable Mass Systems." American Joumal of Physics 50(7): 599-601.

17. Cveticanin, L. (1984). "Vibrations of a Textile Machine Rotor." Journal of Sound and Vibration 97(2): 181-187.

18. Cveticanin, L. (1993). "Conservation Laws in Systems with Variable Mass." ASME Joumal of Applied Mechanics 60(4): 954-958.

19. Davis, L. J., J. W. Follin, et al. (1958). Exterior Ballistics of Rockets. New York, D. Van Nostrand Co., Inc.

20. Dryer, M. (1963). "Equations of Motion for An Arbitrary Body of Variable Mass." Advances in the Astronautical Sciences 9: 804-842. 
21. Duan, C.Y., (1989) 'Dynamics and Applications of Nonholonomic Variable Mass Systems", Proceedings of the International Conference on Applied Mechanics, Beijing, China, p 81-86

22. Eke, F. O. (1982). Star 48 Coning Anomaly. JPL Internal Document No. 343-829891982.

23. Eke, F. O. (1983). Dynamics of Variable Mass Systems with Application to The Star 48 Solid Rocket Motor., AAS 83-370, Advances in the Astronautical Sciences, V 54, p 671-684

24. Eke, F. O. (1991). Angular Momentum and Multibody Dynamics. AAS 91-110, Advances in the Astronautical Sciences, V 75, p 365-376

25. Eke, F. O. and S. M. Wang (1995). “Attitude Behavior of a Variable Mass

Cylinder." ASME Joumal of Applied Mechanics. V62, No. 4 : 935-940:

26. Ellis, J. W. and C. W. McArthur (1959). “Applicability of Euler's Dynamical Equations to Rocket Motion." ARS Journal 29: 863-864.

27. Fang, B. T. (1965). "Kinetic Energy and Angular Momentum about the Variable Center of Mass of a Satellite." AIAA Joumal 3(8): 1540-1542.

28. Fedotkin, I. M., S. M. Konstantinov, et al. (1973). “Separation of a Vapor Bubble and Calculation of the Critical Bubble Radius." Journal of Engineering Physics 24(5): 589592.

29. Flandro, G.A., et al., (1987) 'Fluid Mechanics of Spinning Rockets" AFRPL-TR86-072, Air Force Rocket Propulsion Laboratory, Edwards AFB, California

30. Gadsden, C. P. (1964). "Newton's Law With Variable Mass." American Journal Of Physics 32: 61 .

31. Gadsden, C. P. (1966). "Laws of Motion for Variable Mass Systems." American Journal of Physics 34: 987-988.

32. Gantmacher, F. R. and L. M. Levin (1950). Equations of Motion of a Rocket. NACA TM 1255. 
33. Ge, Z.-M. and Y.-H. Cheng (1983). "The Hamilton's Principle Of Nonholonomic Variable Mass Systems." Applied Mathematics and Mechanics. English Edition 4(2): 291302.

34. Ge, Z. M. (1984). "The Equations of Motion of Nonlinear Nonholonomic Variable Mass System with Application." ASME Journal of Applied Mechanics 51: 435-437.

35. Ge, Z. M. and Y. H. Cheng (1982). "Extended Kane's Equations for

Nonholonomic Variable Mass System." ASME Journal of Applied Mechanics 49: 429431.

36. Gilmore, A. W. and T. L. Keller (1957). "Damping Due to Internal Flow." Journal of the Aeronautical Sciences : 317-318.

37. Gopak, K. M. and O. A. Goroshko (1975). "Vibrations and Stability of a Free Rod of Variable Mass, Taking Account of Motion of Its Mass Along the Axial Line." Soviet Applied Mechanics 11(6): 643-648.

38. Gopak, K. N. (1976). "Dynamic Stability of a Free Rod of Variable Mass." Soviet Applied Mechanics 12(4): 425-428.

39. Grubin, C. (1963). "Mechanics of Variable Mass Systems." Joumal of Eranklin Institute 276: 305-312.

40. Halfman, R. L. (1962). Dynamics. Reading, MA, Addison-Wesley, p 153-170

41. Hammidi, I. B. (1961). The Attitude of a Variable Mass System. Ph.D.

Dissertation, UCLA.

42. Hrushow, W. K. (1969). "Euler's Rotational Equations for Bodies with the Inertia Tensor Varying due to Mass Redistribution and Mass Loss." AIAA Journal 7(2): 337-339. 43. James, R. L. J. (1961). A Three-Dimensional Trajectory Simulation Using Six Degrees of Freedom With Arbitrary Wind. NASA TN D-641.

44. Jarmolow, K. (1957). "Dynamics of a Spinning Rocket with Varying Inertia and Applied Moment." Journal of Applied Physics 28(3): 308-313. 
45. Kane, T. R. and D. A. Levinson (1983). "Multibody Dynamics." ASME Journal of Applied Mechanics 50: 1071-1078.

46. Kane, T. R. and D. A. Levinson (1985). Dynamics: Theory and Applications. McGraw-Hill, Inc.

47. Kapoulitsas, G. (1987). "The Mass-Center Motion of a Continuously Variable System of Particles." Ingenieur-Archiv 57: 91-98.

48. Kayuk, Y. F. and A. A. (1992). "Spatial Motion of a Cylindrical Body of Variable Mass on an Elastic Suspension." Soviet Applied Mechanics 28(7): 460-456.

49. Kayuk, Y. F. and A. Tilavov (1987). "Motion of a Solid of Variable Mass on Elastic Dampers." Soviet Applied Mechanics 23(5): 505-512.

50. Kayuk, Y. f. and A. Tilavov (1990). "Nonlinear Oscillations of a Varying Reservoir." Soviet Applied Mechanics 26(10): 1014-1021.

51. Kayuk, Y. F., A. Tilavov, et al. (1992). “Analysis of Vibrational Motions of a Solid Body of Variable Mass." Soviet Applied Mechanics 28(8): 536-541.

52. Kononov, Y. N. (1986). "Motion of a Rigid Body with a Cylindrical Cavity Partially Filled with an Ideal Fluid of Variable Mass." Mekhanika Tyerdodo Tela 28: 8488.

53. Kosmodemyanskii, A. A. (1963). A Course in Theoretical Mechanics. Jerusalem, Israel Program Scientific Translations. 2nd, ed. p 376-455.

54. Leitmann, G. (1957). "On the Equation of Rocket Motion." Journal of British Interplanetary Society 16: 141-147.

55. Leitmann, G. (1958). "A Remark on the Free Vibration of a Variable Mass." Jet Propulsion 28: 197-198.

56. Leitmann, G. (1963). "On the Stability of Motion of a Vibrating Variable Mass." Astronautica Acta 9: 167-173.

57. Leon, H. I. (1959). "Spin Dynamics of a Thrusting Rocket in Vacuum." Proceedings of the Fourth Midwestern Conference of Solid Mechanics: 514-530. 
58. Marchenko, V. P. and V. M. Podzhio (1966). "Motion of a Body of a Variable Mass Through a Resistant Medium." Soviet Applied Mechanics 2(6): 53-56.

59. Marcinek, J. J. (1958). “On the Free Vibration of a Variable Mass." Let Propulsion 28: $198-199$.

60. Martz, C. W. (1961). Method for Approximating the Vacuum Motions of Spinning Symmetrical Bodies with Nonconstant Spin Rates. NASA TR-115.

61. Matolyak, J. and G. Matous (1990). “Simple Variable Mass Systems: Newton's Second Law." The Physics Teacher 27: 328-329.

62. Matrosov, V. M. (1962). "On the Theory of Stability of Motion." Applied Mathematics and Mechanics 26(6): 1506-1522.

63. McDonald, K. T. (1991). "Motion of a Leaky Tank Car." American Joumal of Physics 59(9): 813-816.

64. McPhee, J. J. (1993). Two Different Methods for Simulating the Motion of Variable-Mass Multibody Systems. Advanced Multibody System Dynamics. Kluwer Academic. $385-390$.

65. Mcphee, J. J. and R. N. Dubey (1991). "Dynamic Analysis and Computer Simulation of Variable-Mass Multi-Rigid-Body Systems." Intemational Joumal for Numerical Methods in Engineering 32: 1711-1715.

66. Mei, F.X. (1989) 'Time Integral Theorem for Variable Mass Systems", Proceedings of the International Conference on Applied Mechanics, Beijing, China, p 102 108

67. Meirovitch, L. (1970). "General Motion of a Variable-Mas̈ Flexible Rocket with Internal Flow." Joumal of Spacecraft and Rocket 7(2): 186-195.

68. Menon, V. J. and D. C. Agrawal (1987). “Newton's Law of Motion for Variable Mass Systems Applied to Capillary." American Journal of Physics 55(1): 63-67. 
69. Meriam, J. L. (1960). “Variable-Mass Dynamics." Joumal of Engineering Education 51(3): 241-243.

70. Meriam, J. L. (1962). "Momentum Equations for Variable Mass." Aerospace Engineering : 52-53,84-87.

71. Meriam, J. L. (1975). Dynamics. John Wiley \& Sons, Inc., p 393-420

72. Meshcherskii, I.V., (1904), "Equations of Motion of a Variable Mass Point in the General Case," St. Petersburg Polytechnic University News, Vol. 1, pp. 77-118 (reprinted in: I.V. Meshcherskii, "Works on the Mechanics of Variable-Mass Bodies," MoscowLeningrad, 1949, 2nd edition, Moscow, 1952).

73. Meyer, R.X., (1983) "Convective Instability in Solid Propellant Rocket Motors", AAS 83-368, Advances in Astronautical Sciences, Vol. 54, p 657-669

74. Mingori, D.L. and Yam, Y., (1986) "Nutational Instability of a Spinning Spacecraft with Internal Mass Motion and Axial Thrust", AIAA paper 86-2271, AIAA/AAS Astrodynamics Conference, Williamsburg, VA, Aug. 18-20, p 367-375

75. Miesse, C. C. (1957). “The Free Vibration of a Variable Mass.” Jet Propulsion 27: 1103-1104.

76. Mikhailov, G. K. (1975). "On the History of Variable Mass System Dynamics." Mechanics of Solids 10(5): $32-40$.

77. Moore, W. (1813). A Treatise on the Motion of Rockets. London, 78. Munson, B. R., D. F. Young, et al. (1994). Eundamentals of Fluid Mechanics. John Wiley \& Sons, Inc.

79. Murphy, C. H. (1981). "Spin Jet Damping of Rocket-Assisted Projectiles." Journal of Guidance and Control 4(3): 350-351.

80. Nita, M. M. (1979). "On the Equations of Motion about the Mass Center of the Jet Aircraft Considered as Variable Mass System." Rev. Roum. Sci. Techn. - Mec. Appl. Tome 24(4): 527-536. 
81. Nita, M. M. and R. Patraulea (1990). "Rotary Damping in Aircraft Motion due to Jet Propulsion System." Rev. Roum. Sci. Techn-Mec. Appl. Tome 35(1): 3-15. 82. Novoselov, V. M. (1959). "Investigation of Stability of Vertical Position of Variable Mass Gyroscope (in Russian)." Yestnik Leningrad Univ 14(19): 121-129. 83. Pan'kin, N. A. (1970). "Motion of a Train in the Start-up Phase (in Russian)." Tr. MIIT (310): 63-72.

84. Pan'kin, N. A. and A. A. Bakman (1984). "Determination of the Rate of Increase of the Thrust in the Start-up Process (in Russian)." TR. MUIT (758): 48-52.

85. Papis, T. and C. W. Bullard (1968). "Attitude of a Spinning Rocket under Thrust with Statistically Varied Error Sources." Journal of Spacecraft and Rocket 5(5):

86. Petrenko, M. P. (1968). "Oscillations in a System of Variable-Mass Material Points." Soviet Applied Mechanics 4(6): 64-67.

87. Poisson, S.D., (1819), "Sur le Mouvement d'un System de Corps, en Supposant les Masses Variables, Bull. Sci. Soc. Philomat, April, pp. 60-62, Paris.

88. Polotskii, V. N. (1990). "Some Problems of Dynamics Bodies of Variable Mass in the Mechanics of Coupled Trains." Mechanics of Solids 25(2): 73-82.

89. Pomeranz, K. B. (1964). "Newton's Law for Systems with a Variable Mass." American Joumal of Physics 32(5): 386.

90. Prato, D. and R. J. Gleiser (1982). "Another Look at the Uniform Rope Sliding over the Edge of a Smooth Table." American Joumal of Physics 50(6): 536-539.

91. Punga, V. (1963). "Lagrangian and Hamiltonian Rocket Mechanics." AIAA Joumal 1(3): 709-711.

92. Punga, V. (1964). "Euler's Moment Equations for a Variable-Mass Unsymmetrical Top." ALAA Joumal 2(5): 937-938.

93. Punga, V. (1964). "Motion of the Center of Gravity of a Variable-Mass Body." AIAA Journal 2(8): 1482. 
94. Rankin, R. A. (1949). “The Mathematical Theory of the Motion of Rotated and Unrotated Rockets." Philosophical Transactions Royal Society London Series A 241: 457 585.

95. Rosser, J. B., R. R. Newton, et al. (1947). Mathematical Theory of Rocket Elight. New York, McGraw-Hill.

96. Rott, N. and L. Pottsepp (1964). "Simplified Calculation of the Jet Damping Effects." AIAA Journal 2(4): 764-766.

97. Shomin, R. (1992). “A Special Variable Mass Dynamics Problem.” The Physics Teacher 30: 304-305.

98. Shul'gin, A. M. and A. S. Andreev (1979). "Stabilization of an Equilibrium Position of a Solid body with Variable Moments of Inertia in its Motion About the Center of Mass." Differential Equations 15(5): 677-680.

99. Sibert, H. W. (1953). "Relation of Force, Acceleration, and Momentum for a Body Whose Mass is Varying." Joumal of the Aeronautical Sciences : 141-142.

100. Siegel, S. (1972). "More about Variable Mass systems.” American Journal of Physics 40: 183-185.

101. Silli, C. (1965). "Sulla Stabilita Dei Missili Con Massa Variable." Boll. Un. Mat. Ital. Ser. 3 20: 194-209.

102. Smotrov, V. M. and V. M. Chernyshev (1972). "Equations of Oscillations of Rods of Variable Composition." Joumal of Applied Mechanics and Technical Physics 13(1): 90 94.

103. Smotrov, V. M. and V. M. Chemyshev (1973). "Vibrational Equations of Variable Mass Layers and Membranes." Loumal of Applied Mechanics and Technical Physics 14(5): 715-719.

104. Snyder, V. W. (1964). "Comment on “An Alternate Interpretation of Newton's Second Law"." AIAA Joumal 2: 2239-2240. 
105. Snyder, V. W. (1965). "Comment on "Motion of the Center of Gravity of a 106 Squire, W. (1971). “An Example Motion

American Joumal of Phics 39.1281. 107. Tait, P.G., and Steele, W.J., (1856), "A Treatise on the Dynamics of a Particle,"
Cambridge.

108. Tempelman, W. H. (1972). "Equations of Motion for the Variable Mass FlowVariable Exhaust Velocity Rocket." ALAANo. 72-912:

109. Thomson, W. T. (1966). "Equations of Motion for the Variable Mass System." 110. Thomson, W. T. (1986). Introduction to Space Dynamics. New York, Dover. 111. Thomson, W. T. and G. S. Reiter (1965). "Jet Damping of a Solid Rocket: Theory and Flight Results." ALAA Joumal 3(3): 413-417. Variable Mass." American Joumal of Physics 30: 637-640.

113. Tiersten, M. S. (1969). "Force, Momentum Change, and Motion." American Journal of Physics 37(1): 82-87.

114. Trigg, G. L. (1966). "Laws of Motion." American Joumal of Physics 34(1): 71. 115. Van den Akker, J. A. (1964). "Newton's Law with Variable Mass." American Journal of Physics 32: 387.

116. Wang, S. M. (1993). Artinde Dynamics of Variable Mass Systems. Ph.D.

Dissertation, University of Califomia, Davis, September.

117. Wang S.M. and Eke, F. O. (1995). "Rotational Dynamics of Axisymmetric Variable Mass Systems." ASME Journal of Applied Mechanics, V62, No. 4 : 970-974 118. Warner, G. G. (1968). "A Re-Evaluation of Jet Damping." Lournal Spacecraft and Rocket 5(3): 364-366. 
119. Wu, M.-S. (1986). "Note on a Conveyor-belt Problem." The Physics Teacher 24:

220. 